\title{
ELASTIC FLOW WITH JUNCTIONS: \\ VARIATIONAL APPROXIMATION AND APPLICATIONS TO NONLINEAR SPLINES
}

\author{
JOHN W. BARRETT \\ Department of Mathematics, Imperial College London, London, SW7 2AZ, UK \\ j.barrett@imperial.ac.uk \\ HARALD GARCKE \\ Fakult" at f ur Mathematik, Universit"at Regensburg, 93040 Regensburg, Germany \\ barald.garcke@mathematik.uni-regensburg.de \\ ROBERT N"URNBERG \\ Department of Mathematics, Imperial College London, London, SW7 2AZ, UK \\ robert.nurnberg@imperial.ac.uk. \\ Received (10 August 2011) \\ Revised (25 January 2012) \\ Communicated by (xxxxxxxxxx)
}

\begin{abstract}
We consider stable semidiscrete approximations of parameterized curve networks for gradient flows of elastic type functionals. Here meaningful and relevant conditions at junction points, such as double and triple junctions, need to be derived and suitably discretized. Examples for double junction types are $\mathrm{C} 0$ attachment and $\mathrm{C} 1$ continuity. We develop strong and weak formulations for the elastic flow for curve networks with such junction points. For junctions with three or more curves the conditions at the junctions are derived here for the first time. Possible applications include a simplified one-dimensional model of geometric biomembranes, as well as nonlinear splines in two and higher dimensions. The numerical results presented in this paper demonstrate the practicality of the introduced finite element approximations.
\end{abstract}

Keywords: Elastic flow; Willmore flow; parametric finite elements; tangential movement; curve networks; junctions; nonlinear splines.

\section{Introduction}

In many applications in elasticity theory, computer vision, shape reconstruction or in problems which require the interpolation of points one is interested in minimizing the integrated squared curvature of curves.8,27,24,25 Typically side constraints leading to boundary conditions at junctions have to be imposed in these problems. For example, in interpolation theory one is interested in finding a curve passing through 
an ordered set of points in Euclidean space in a smooth way. It was first proposed by Birkhoff and de Boor ${ }^{8}$ to minimize the curvature energy of a such a curve. This approach is motivated by the traditional mechanical splines of draftsmen, and these so called nonlinear splines have been studied by many authors. ${ }^{8,21,15,23,24,22,19}$ In other applications, e.g. in simplified models for a biological membrane, two phases appear on the curve and certain physical parameters, such as bending coefficients or intrinsic curvatures, undergo jump discontinuities. This will also lead to junction conditions at a phase boundary. Finally, we are also interested in curve networks with junctions at which more than two curves meet - a situation relevant for elastic networks with junctions.

For all of the above situations we will derive equilibrium conditions at the junctions, and we will also consider gradient flows of the underlying energies. A major part of the paper is devoted to the derivation of numerical schemes for the geometric partial differential equations governing the evolution of the networks. Continuous in time semidiscrete versions of the schemes fulfill an equidistribution property and can be shown to be stable, i.e. they satisfy a discrete analogue of the gradient flow property of the continuous problem. To establish the latter is highly non-trivial due to the highly nonlinear character of the problem. We now introduce some notation in order to specify the problem.

Let $\vec{x}(\rho, t): \mathbb{R} / \mathbb{Z} \times[0, T] \rightarrow \mathbb{R}^{d}, d \geq 2$, be the parameterization of an evolving closed curve. The standard elastic energy is given by

$$
E_{\lambda}(\Gamma, \vec{\varkappa})=\frac{1}{2} \int_{\Gamma}\left(|\vec{\imath}|^{2}+2 \lambda\right) \mathrm{d} s
$$

where

$$
\vec{\varkappa}=\vec{x}_{s s}=\frac{1}{\left|\vec{x}_{\rho}\right|}\left(\frac{\vec{x}_{\rho}}{\left|\vec{x}_{\rho}\right|}\right)_{\rho}
$$

denotes the curvature vector of $\Gamma$, with $s$ being arclength.

We define $\vec{P}:=\overrightarrow{I d}-\vec{x}_{s} \otimes \vec{x}_{s}$, the projection onto the part normal to $\Gamma$. Here $\overrightarrow{I d}$ is the identity operator on $\mathbb{R}^{d}$. Moreover, let $\vec{\nabla}_{s} \vec{\eta}:=\vec{P} \vec{\eta}_{s}$ be the normal component of $\vec{\eta}_{s}$, and $\vec{\nabla}_{s}^{2} \cdot:=\vec{\nabla}_{s}\left(\vec{\nabla}_{s} \cdot\right)$.

The $L^{2}$-gradient flow of (1.1) is given as ${ }^{12,4}$

$$
\vec{P} \vec{x}_{t}=-\left(\vec{\nabla}_{s} \vec{\varkappa}\right)_{s}-\frac{1}{2}\left(|\vec{\varkappa}|^{2} \vec{x}_{s}\right)_{s}+\lambda \vec{\varkappa}=-\vec{\nabla}_{s}^{2} \vec{\varkappa}-\frac{1}{2}|\vec{\varkappa}|^{2} \vec{\varkappa}+\lambda \vec{\varkappa} .
$$

In particular, a curve parameterized by a solution to (1.3) satisfies

$$
\frac{\mathrm{d}}{\mathrm{d} t} E_{\lambda}(\Gamma, \vec{\varkappa})=-\int_{\Gamma}\left|\vec{P} \vec{x}_{t}\right|^{2} \mathrm{~d} s \leq 0 .
$$

In the recent paper Ref. 7, the present authors combined ideas from Ref. 10, Ref. 4 and the formal calculus of PDE constrained optimization, ${ }^{26}$ in order to derive a finite element approximation of the elastic flow (1.3) based on the following weak formulation of this gradient flow. Given $\Gamma(0)$, for all $t \in(0, T]$ find $\Gamma(t)=\vec{x}(\mathbb{R} / \mathbb{Z}, t)$, 
where $\vec{x}(t) \in \underline{V}_{0}:=H^{1}\left(\mathbb{R} / \mathbb{Z}, \mathbb{R}^{d}\right)$, and $\vec{y}(t) \in \underline{V}_{0}$ such that

$$
\begin{aligned}
& \left\langle\vec{P} \vec{x}_{t}, \vec{\chi}\right\rangle_{\Gamma}-\left\langle\vec{\nabla}_{s} \vec{y}, \vec{\nabla}_{s} \vec{\chi}\right\rangle_{\Gamma}-\frac{1}{2}\left\langle|\vec{P} \vec{y}|^{2} \vec{x}_{s}, \vec{\chi}_{s}\right\rangle_{\Gamma}+\left\langle\left(\vec{y} \cdot \vec{x}_{s}\right) \vec{P} \vec{y}, \vec{\chi}_{s}\right\rangle_{\Gamma}=0 \quad \forall \vec{\chi} \in \underline{V}_{0}, \\
& \langle\vec{P} \vec{y}, \vec{\eta}\rangle_{\Gamma}+\left\langle\vec{x}_{s}, \vec{\eta}_{s}\right\rangle_{\Gamma}=0 \quad \forall \vec{\eta} \in \underline{V}_{0},
\end{aligned}
$$

where $\langle\cdot, \cdot\rangle_{\Gamma}$ denotes the $L^{2}$-inner product on $\Gamma$. Here $\vec{y}$ formally is a Lagrange multiplier for the equation (1.2), and it can be shown that

$$
\vec{y}=\vec{\varkappa}+\left(\vec{y} \cdot \vec{x}_{s}\right) \vec{x}_{s} \quad \Rightarrow \quad \vec{\varkappa}=\vec{P} \vec{y} .
$$

Hence it can be shown that a solution to (1.5a), (1.5b) satisfies

$$
\frac{\mathrm{d}}{\mathrm{d} t} E_{\lambda}(\Gamma, \vec{P} \vec{y})=-\int_{\Gamma}\left|\vec{P} \vec{x}_{t}\right|^{2} \mathrm{~d} s \leq 0 .
$$

In Ref. 7 the authors introduced a continuous-in-time finite element approximation of $(1.5 \mathrm{a}),(1.5 \mathrm{~b})$ which satisfies a discrete analogue of (1.7) and which yields spatially uniform discretizations. The latter property makes this approximation particularly appealing from a practical point of view, as the fully discrete finite element schemes need no remeshing procedure and are free from coalescence. The introduced schemes share this equidistribution property with a number of other approximations derived by the authors for the approximation of geometric evolution equations over the last few years. ${ }^{2,1,3,4,6}$ For an overview on the numerical approximation of geometric evolution equations we refer to the survey article Ref. 11.

Apart from simple closed curves, in Ref. 7 the authors also considered the elastic flow for open curves. Here the flow equation (1.3) in the interior of the curve needs to be coupled with appropriate boundary conditions at the two endpoints, in order to still satisfy the gradient flow property (1.4). Physically relevant boundary conditions are so-called clamped conditions, where position and angle(s) are fixed, and so-called Navier conditions, where position and curvature is fixed. We would like to stress that the finite element approximations in Ref. 7 for the open curve case were the first variational numerical methods for the elastic flow of open curves with boundary conditions.

It is the aim of the present article to generalize the ideas from Ref. 7 on the approximation of the elastic flow of open curves to more general curve networks with junctions. Here we will consider open and closed chains of curves with double junctions and possible boundary points, as well as higher order junctions involving three or more curves meeting at a point. See Figure 1 for a sketch of possible situations. The types of junctions considered in this paper are for two curves: $C^{0}$ junction, $C^{1}$ junction and $C^{1}$ spline junction. For more than two curves we consider junctions with the following properties: attachment only ("C $C^{0 ")}$, attachment and Young's law (" $C^{1}$ "), fixed in space and Young's law (" $C^{1}$ spline"). In each case the boundary conditions for $\Gamma$ at the junctions need to be formulated, so that the resulting flow still satisfies (1.4). Moreover, weak formulations need to be derived, which will then form the basis for the finite element approximations. Building on the 

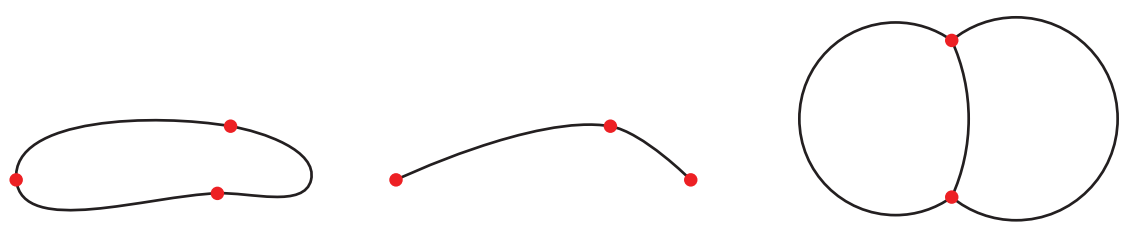

Fig. 1. Closed chain with $N=3$ curves, open chain with $N=2$, and a triple junction network with $N=3$.

work in Ref. 7, it is often straightforward to formulate the desired weak formulations, while deriving and interpreting the corresponding strong boundary conditions is much harder.

In preparation for later considerations we note that in the planar case, $d=2$, the curvature $\varkappa$ of $\Gamma$ can be defined via $\vec{\varkappa}=\varkappa \vec{\nu}$ with $\vec{\nu}:=-\vec{x}_{s}^{\perp}$ and $\cdot^{\perp}$ acting on a vector in $\mathbb{R}^{2}$ denoting a clockwise rotation through $90^{\circ}$. Then the elastic energy (1.1) is equivalent to

$$
E_{\lambda}(\Gamma, \varkappa)=\frac{1}{2} \int_{\Gamma}\left(\varkappa^{2}+2 \lambda\right) \mathrm{d} s .
$$

Moreover, it is then possible to introduce a so-called spontaneous curvature $\bar{\varkappa} \in \mathbb{R},{ }^{16}$ and consider the energy

$$
E_{\bar{\varkappa}}(\Gamma, \varkappa)=\frac{1}{2} \int_{\Gamma}(\varkappa-\bar{\varkappa})^{2} \mathrm{~d} s
$$

instead. It was discussed in Ref. 7 that the gradient flows for (1.8) and (1.9) for simple closed curves are equivalent if $\lambda=\frac{1}{2} \bar{\varkappa}^{2}$. Hence for closed curves there is no need to consider an energy of the form (1.9). However, the situation is different for an open curve that forms part of a network of curves. Here $\bar{\varkappa}$ and $\lambda$ will play different roles during the evolution, and with a view towards physically and biomathematically interesting and relevant simulations, it is crucial to consider energies of the form (1.9).

We remark that so far there is little work on the numerical approximation of the elastic flow for junctions. In Ref. 13 a thresholding algorithm is used in order to evolve a curve network via (1.3) for $d=2$. However, no considerations to boundary conditions are made and so a decrease of the elastic energy similar to (1.4) cannot be expected. In addition, in Ref. 20 a phase-field model of a situation where two curves, attached to each other with two double junctions, move by a gradient flow for a biologically motivated generalized elastic energy, is considered. A similar model is considered in Ref. 17.

The remainder of the paper is organized as follows. In Section 2 we present in detail the conditions at the curve junctions that we consider in this paper together with their strong and weak formulations. We also establish that together with the flow equation (1.3) on the curves, these junction conditions lead to an 
$L^{2}$-gradient flow of the considered elastic energy. In Section 3 we present a semidiscrete continuous-in-time finite element approximation of the elastic flow for curve networks with junctions together with a stability result. The corresponding fully discrete scheme and ways to solve the resulting discrete systems of equations are presented in Sections 4 and 5. Finally, we show numerous numerical simulations in Section 6 .

\section{Variational formulation for the elastic flow of junctions}

In this section we derive the junction conditions that need to hold in a network of curves in order for an elastic energy to decrease in time for the elastic flow. Let $\vec{x}_{i}(\rho, t):[0,1] \times[0, T] \rightarrow \mathbb{R}^{d}$ be the parameterizations of $N$ evolving open curves $\Gamma_{i}$, $i=1 \rightarrow N$. The curvature vectors are then defined by

$$
\vec{\varkappa}_{i}=\vec{x}_{i, s s}, \quad i=1 \rightarrow N .
$$

Let $\Gamma=\left(\Gamma_{1}, \ldots, \Gamma_{N}\right), \vec{\varkappa}=\left(\vec{\varkappa}_{1}, \ldots, \vec{\varkappa}_{N}\right)$ and consider the energy

$$
E_{\lambda}(\Gamma, \vec{\varkappa})=\frac{1}{2} \sum_{i=1}^{N} \varsigma_{i} \int_{\Gamma_{i}}\left(\left|\vec{\varkappa}_{i}\right|^{2}+2 \lambda_{i}\right) \mathrm{d} s,
$$

where we use the notations $\lambda=\left(\lambda_{1}, \ldots, \lambda_{N}\right) \in \mathbb{R}^{N}, \varsigma=\left(\varsigma_{1}, \ldots, \varsigma_{N}\right) \in \mathbb{R}_{>0}^{N}$, and so on, here and throughout the paper. In addition, in the case $d=2$ we consider the energy

$$
E_{\lambda, \bar{\varkappa}}(\Gamma, \varkappa)=\frac{1}{2} \sum_{i=1}^{N} \varsigma_{i} \int_{\Gamma_{i}}\left(\left(\varkappa_{i}-\bar{\varkappa}_{i}\right)^{2}+2 \lambda_{i}\right) \mathrm{d} s,
$$

where $\bar{\varkappa}=\left(\bar{\varkappa}_{1}, \ldots, \bar{\varkappa}_{N}\right) \in \mathbb{R}^{N}$ are given spontaneous curvatures.

In order to keep the initial presentation simple, from now on we consider a network of $N$ curves that all meet at two junction points. We also assume that each curve is oriented in the same way, so that they all start and end at the same points. Generalizations to more complex topologies are straightforward.

For this simplified setup, we consider the following types of junctions. For $r \in$ $\{0,1\}$ and for all $t \geq 0$ we require one of the following.

$$
\begin{array}{ll}
C^{0} \text { junction } & \begin{cases}\vec{x}_{i}(r, t)=\vec{x}_{1}(r, t), & i=2 \rightarrow N ;\end{cases} \\
C^{1} \text { junction } & \begin{cases}\vec{x}_{i}(r, t)=\vec{x}_{1}(r, t), & i=2 \rightarrow N, \\
\sum_{i=1}^{N} \vec{x}_{i, s}(r, t)=\overrightarrow{0} ;\end{cases} \\
C^{1} \text { spline node }\left\{\begin{array}{l}
\vec{x}_{i}(r, t)=\vec{\alpha}_{r}, \\
\sum_{i=1}^{N} \vec{x}_{i, s}(r, t)=\overrightarrow{0} ;
\end{array}\right.
\end{array}
$$




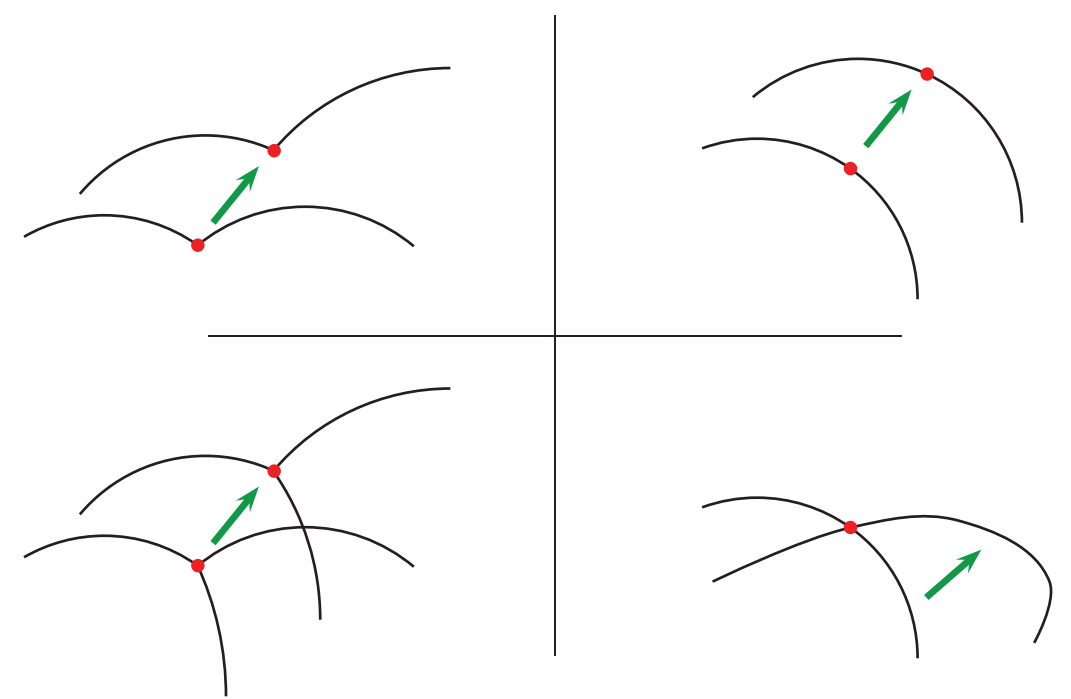

Fig. 2. Different types of junctions (clock-wise from top-left): a moving $C^{0}$ double junction, a moving $C^{1}$ double junction, a stationary $C^{1}$ spline double junction and a moving " $C^{1}$ " triple junction.

where $\vec{\alpha}_{0}, \vec{\alpha}_{1} \in \mathbb{R}^{d}$ are given "spline" interpolation points. Here the terms " $C^{k}$ junction", for $k=0 \rightarrow 1$, and " $C^{1}$ spline node" really only make sense for $N=2$, when exactly two curves meet at the two junction points. However, for ease of presentation we will use the same terms, in an abuse of notation, also for the cases $N \geq 3$. Some example junctions for $N=2$ and $N=3$ are presented in Figure 2.

In what follows we will derive suitable junction conditions to complement (2.4a)(2.4c) for the $L^{2}$-gradient flows of $(2.2)$ and $(2.3)$. Let $\langle\cdot, \cdot\rangle_{\Gamma_{i}}$ denote the $L^{2}$-inner product on $\Gamma_{i}$; that is, $\langle u, v\rangle_{\Gamma_{i}}:=\int_{0}^{1} u . v\left|\vec{x}_{i, \rho}\right| \mathrm{d} \rho, i=1 \rightarrow N$.

Lemma 2.1. Let $\vec{x}_{i}(\rho, t):[0,1] \times[0, T] \rightarrow \mathbb{R}^{d}$ be a parameterization of the open curve $\Gamma_{i}(t)=\vec{x}_{i}([0,1], t)$. It holds that

$$
\frac{\mathrm{d}}{\mathrm{d} t} \frac{1}{2}\left\langle\left|\vec{\varkappa}_{i}\right|^{2}, 1\right\rangle_{\Gamma_{i}}=\left\langle\vec{\nabla}_{s}^{2} \vec{\varkappa}_{i}+\frac{1}{2}\left|\vec{\varkappa}_{i}\right|^{2} \vec{\varkappa}_{i}, \overrightarrow{\mathcal{V}}_{i}\right\rangle_{\Gamma_{i}}+\left[\frac{1}{2}\left|\vec{\varkappa}_{i}\right|^{2} v_{i}-\vec{\varkappa}_{i, s} \cdot \overrightarrow{\mathcal{V}}_{i}+\vec{\varkappa}_{i} \cdot \vec{\nabla}_{s} \overrightarrow{\mathcal{V}}_{i}\right]_{0}^{1},
$$

where $\overrightarrow{\mathcal{V}}_{i}:=\vec{P}_{i} \vec{x}_{i, t}$ and $\vec{x}_{i, t}=\overrightarrow{\mathcal{V}}_{i}+v_{i} \vec{x}_{i, s}$. Similarly, in the case $d=2$ it holds that

$$
\begin{aligned}
\frac{\mathrm{d}}{\mathrm{d} t} \frac{1}{2}\left\langle\left(\varkappa_{i}-\bar{\varkappa}_{i}\right)^{2}, 1\right\rangle_{\Gamma_{i}}=\left\langle\varkappa_{i, s s}+\frac{1}{2}\left(\varkappa_{i}^{2}-\bar{\varkappa}_{i}^{2}\right) \varkappa_{i}, \mathcal{V}_{i}\right\rangle_{\Gamma_{i}} \\
+\left[\frac{1}{2}\left(\varkappa_{i}-\bar{\varkappa}_{i}\right)^{2} v_{i}-\varkappa_{i, s} \mathcal{V}_{i}+\left(\varkappa_{i}-\bar{\varkappa}_{i}\right) \mathcal{V}_{i, s}\right]_{0}^{1},
\end{aligned}
$$

where $\mathcal{V}_{i}:=\vec{x}_{i, t} \cdot \vec{\nu}_{i}$

Proof. The results (2.5a), (2.5b) immediately follow from the proof of Lemma 2.2 in Ref. 7. We note that there the boundary terms involving $v_{i}, \overrightarrow{\mathcal{V}}_{i}$ and $\mathcal{V}_{i}$ were dropped due to the assumed stationary boundary conditions on $\Gamma_{i}$. 
It follows from $(2.5 \mathrm{a}),(2.5 \mathrm{~b})$ that, together with suitable boundary conditions, the flows

$$
\begin{array}{r}
\varsigma_{i}^{-1} \vec{P}_{i} \vec{x}_{i, t}=-\left(\vec{\nabla}_{s} \vec{\varkappa}_{i}\right)_{s}-\frac{1}{2}\left(\left|\vec{\varkappa}_{i}\right|^{2} \vec{x}_{i, s}\right)_{s}+\lambda_{i} \vec{\varkappa}_{i} \equiv-\vec{\nabla}_{s}^{2} \vec{\varkappa}_{i}-\frac{1}{2}\left(\left|\vec{\varkappa}_{i}\right|^{2}-2 \lambda_{i}\right) \vec{\varkappa}_{i} \\
\text { in }(0,1) \times(0, T), \quad i=1 \rightarrow N ; \text { (2.6a) }
\end{array}
$$

and, if $d=2$,

$$
\varsigma_{i}^{-1} \vec{x}_{i, t} \cdot \vec{\nu}_{i}=-\varkappa_{i, s s}-\frac{1}{2}\left(\varkappa_{i}^{2}-\bar{\varkappa}_{i}^{2}-2 \lambda_{i}\right) \varkappa_{i} \quad \text { in }(0,1) \times(0, T), \quad i=1 \rightarrow N \text {; }
$$

represent $L^{2}$-gradient flows for the energies $E_{\lambda}(\Gamma, \vec{\varkappa})$ and $E_{\lambda, \bar{\varkappa}}(\Gamma, \varkappa)$, respectively. The following theorems make the suitable boundary conditions precise, and they all hinge on the following lemma.

Lemma 2.2. Let $N \geq 2$ and let $\vec{x}(\rho, t):[0,1] \times[0, T] \rightarrow\left(\mathbb{R}^{d}\right)^{N}$ be a parameterization of the curve network $\Gamma(t)=\vec{x}([0,1], t)$. Let

$$
\vec{x}_{i}(r, t)=\vec{x}_{1}(r, t), \quad i=2 \rightarrow N, \quad r \in\{0,1\} .
$$

Let $\lambda, \bar{\varkappa} \in \mathbb{R}^{N}$ and $\varsigma \in \mathbb{R}_{>0}^{N}$. Then it holds that

$$
\begin{aligned}
& \frac{\mathrm{d}}{\mathrm{d} t} E_{\lambda}(\Gamma, \vec{\varkappa})-\sum_{i=1}^{N} \varsigma_{i}\left\langle\vec{\nabla}_{s}^{2} \vec{\varkappa}_{i}+\frac{1}{2}\left(\left|\vec{\varkappa}_{i}\right|^{2}-\lambda_{i}\right) \vec{\varkappa}_{i}, \overrightarrow{\mathcal{V}}_{i}\right\rangle_{\Gamma_{i}} \\
& \quad=\left[\vec{x}_{1, t} \cdot \sum_{i=1}^{N} \frac{1}{2} \varsigma_{i}\left(2 \lambda_{i}-\left|\vec{\varkappa}_{i}\right|^{2}\right) \vec{x}_{i, s}\right]_{0}^{1}-\left[\vec{x}_{1, t} \cdot \sum_{i=1}^{N} \varsigma_{i} \vec{\nabla}_{s} \vec{\varkappa}_{i}\right]_{0}^{1}+\sum_{i=1}^{N}\left[\varsigma_{i} \vec{\varkappa}_{i} \cdot\left(\vec{x}_{i, s}\right)_{t}\right]_{0}^{1} .
\end{aligned}
$$

Moreover, in the case $d=2$ it holds that

$$
\begin{aligned}
\frac{\mathrm{d}}{\mathrm{d} t} E_{\lambda, \bar{\varkappa}}(\Gamma, \varkappa)- & \sum_{i=1}^{N} \varsigma_{i}\left\langle\varkappa_{i, s s}+\frac{1}{2}\left(\varkappa_{i}^{2}-\bar{\varkappa}_{i}^{2}-2 \lambda_{i}\right) \varkappa_{i}, \mathcal{V}_{i}\right\rangle_{\Gamma_{i}} \\
= & {\left[\vec{x}_{1, t} \cdot \sum_{i=1}^{N} \frac{1}{2} \varsigma_{i}\left(\bar{\varkappa}_{i}^{2}-\varkappa_{i}^{2}+2 \lambda_{i}\right) \vec{x}_{i, s}\right]_{0}^{1} } \\
& -\left[\vec{x}_{1, t} \cdot \sum_{i=1}^{N} \varsigma_{i} \varkappa_{i, s} \vec{\nu}_{i}\right]_{0}^{1}+\sum_{i=1}^{N}\left[\varsigma_{i}\left(\varkappa_{i}-\bar{\varkappa}_{i}\right) \vec{\nu}_{i} \cdot\left(\vec{x}_{i, s}\right)_{t}\right]_{0}^{1} .
\end{aligned}
$$

Proof. The following results are easily derived, see e.g. Lemma 2.1 in Ref. 12:

$\left|\vec{x}_{i, \rho}\right|_{t}=\left(v_{i, s}-\vec{\varkappa}_{i} \cdot \overrightarrow{\mathcal{V}}_{i}\right)\left|\vec{x}_{i, \rho}\right|, \partial_{t} \partial_{s}-\partial_{s} \partial_{t}=\left(\vec{\varkappa}_{i} \cdot \overrightarrow{\mathcal{V}}_{i}-v_{i, s}\right) \partial_{s},\left(\vec{x}_{i, s}\right)_{t}=\vec{\nabla}_{s} \overrightarrow{\mathcal{V}}_{i}+v_{i} \vec{\varkappa}_{i}$,

where the last equality can equivalently be written as

$$
\left(\vec{x}_{i, s}\right)_{t}=\vec{P}_{i}\left[\vec{x}_{i, t}\right]_{s}=\vec{\nabla}_{s} \vec{x}_{i, t} .
$$


It immediately follows from (2.9) that

$$
\frac{\mathrm{d}}{\mathrm{d} t}\left|\Gamma_{i}\right|=\left\langle v_{i, s}-\vec{\varkappa}_{i} \cdot \overrightarrow{\mathcal{V}}_{i}, 1\right\rangle_{\Gamma_{i}}=-\left\langle\vec{\varkappa}_{i}, \overrightarrow{\mathcal{V}}_{i}\right\rangle_{\Gamma_{i}}+\left[v_{i}\right]_{0}^{1}
$$

Hence it follows from (2.5a), (2.11), (2.9) and the attachment conditions (2.7) that

$$
\begin{aligned}
& \frac{\mathrm{d}}{\mathrm{d} t} E_{\lambda}(\Gamma, \vec{\varkappa})-\sum_{i=1}^{N} \varsigma_{i}\left\langle\vec{\nabla}_{s}^{2} \vec{\varkappa}_{i}+\frac{1}{2}\left(\left|\vec{\varkappa}_{i}\right|^{2}-\lambda_{i}\right) \vec{\varkappa}_{i}, \overrightarrow{\mathcal{V}}_{i}\right\rangle_{\Gamma_{i}} \\
& =\sum_{i=1}^{N}\left[\frac{1}{2} \varsigma_{i}\left(\left|\vec{\varkappa}_{i}\right|^{2}+2 \lambda_{i}\right) v_{i}-\varsigma_{i} \vec{\varkappa}_{i, s} \cdot \overrightarrow{\mathcal{V}}_{i}+\varsigma_{i} \vec{\varkappa}_{i} \cdot \vec{\nabla}_{s} \overrightarrow{\mathcal{V}}_{i}\right]_{0}^{1} \\
& =\sum_{i=1}^{N}\left[\frac{1}{2} \varsigma_{i}\left(2 \lambda_{i}-\left|\vec{\varkappa}_{i}\right|^{2}\right) v_{i}-\varsigma_{i} \vec{\nabla}_{s} \vec{\varkappa}_{i} \cdot \vec{x}_{i, t}+\varsigma_{i} \vec{\varkappa}_{i} \cdot\left(\vec{x}_{i, s}\right)_{t}\right]_{0}^{1}, \\
& =\left[\vec{x}_{1, t} \cdot \sum_{i=1}^{N} \frac{1}{2} \varsigma_{i}\left(2 \lambda_{i}-\left|\vec{\varkappa}_{i}\right|^{2}\right) \vec{x}_{i, s}\right]_{0}^{1}-\left[\vec{x}_{1, t} \cdot \sum_{i=1}^{N} \varsigma_{i} \vec{\nabla}_{s} \vec{\varkappa}_{i}\right]_{0}^{1}+\sum_{i=1}^{N}\left[\varsigma_{i} \vec{\varkappa}_{i} \cdot\left(\vec{x}_{i, s}\right)_{t}\right]_{0}^{1},
\end{aligned}
$$

which yields the desired result (2.8a). Similarly, in the case $d=2$ it follows from $(2.5 \mathrm{~b}),(2.11),(2.10)$ and the attachment conditions (2.7) that

$$
\begin{aligned}
\frac{\mathrm{d}}{\mathrm{d} t} E_{\lambda, \bar{\varkappa}}(\Gamma, \varkappa)- & \sum_{i=1}^{N} \varsigma_{i}\left\langle\varkappa_{i, s s}+\frac{1}{2}\left(\varkappa_{i}^{2}-\bar{\varkappa}_{i}^{2}-2 \lambda_{i}\right) \varkappa_{i}, \mathcal{V}_{i}\right\rangle_{\Gamma_{i}} \\
= & \sum_{i=1}^{N}\left[\frac{1}{2} \varsigma_{i}\left[\left(\varkappa_{i}-\bar{\varkappa}_{i}\right)^{2}+2 \lambda_{i}\right] v_{i}-\varsigma_{i} \varkappa_{i, s} \mathcal{V}_{i}+\varsigma_{i}\left(\varkappa_{i}-\bar{\varkappa}_{i}\right) \mathcal{\nu}_{i, s}\right]_{0}^{1} \\
= & {\left[\vec{x}_{1, t} \cdot \sum_{i=1}^{N} \frac{1}{2} \varsigma_{i}\left[\left(\varkappa_{i}-\bar{\varkappa}_{i}\right)^{2}-2\left(\varkappa_{i}-\bar{\varkappa}_{i}\right) \varkappa_{i}+2 \lambda_{i}\right] \vec{x}_{i, s}\right]_{0}^{1} } \\
& -\sum_{i=1}^{N}\left[\varsigma_{i} \varkappa_{i, s} \vec{\nu}_{i} \cdot \vec{x}_{i, t}\right]_{0}^{1}+\sum_{i=1}^{N}\left[\varsigma_{i}\left(\varkappa_{i}-\bar{\varkappa}_{i}\right) \vec{\nu}_{i} \cdot\left(\vec{x}_{i, s}\right)_{t}\right]_{0}^{1} \\
= & {\left[\vec{x}_{1, t} \cdot \sum_{i=1}^{N} \frac{1}{2} \varsigma_{i}\left(\bar{\varkappa}_{i}^{2}-\varkappa_{i}^{2}+2 \lambda_{i}\right) \vec{x}_{i, s}\right]_{0}^{1} } \\
& -\left[\vec{x}_{1, t} \cdot \sum_{i=1}^{N} \varsigma_{i} \varkappa_{i, s} \vec{\nu}_{i}\right]_{0}^{1}+\sum_{i=1}^{N}\left[\varsigma_{i}\left(\varkappa_{i}-\bar{\varkappa}_{i}\right) \vec{\nu}_{i} \cdot\left(\vec{x}_{i, s}\right)_{t}\right]_{0}^{1} .
\end{aligned}
$$

Hence (2.8b) holds.

Theorem 2.1. Let the assumptions of Lemma 2.2 hold. Then the flow (2.6a), with 
the $C^{0}$ junction conditions

$$
\begin{aligned}
\vec{x}_{i}(r, t) & =\vec{x}_{1}(r, t), \quad i=2 \rightarrow N, \\
\sum_{i=1}^{N} \varsigma_{i}\left(\vec{\nabla}_{s} \overrightarrow{\mathcal{x}}_{i}(r, t)-\lambda_{i} \vec{x}_{i, s}(r, t)\right) & =\overrightarrow{0}, \\
\vec{\varkappa}_{i}(r, t) & =\overrightarrow{0}, \quad i=1 \rightarrow N,
\end{aligned}
$$

for $r \in\{0,1\}$ is an $L^{2}$-gradient flow of $E_{\lambda}(\Gamma, \vec{\varkappa})$. Similarly, in the case $d=2$, the flow (2.66) together with the $C^{0}$ junction conditions

$$
\begin{aligned}
\vec{x}_{i}(r, t) & =\vec{x}_{1}(r, t), \quad i=2 \rightarrow N, \\
\sum_{i=1}^{N} \varsigma_{i}\left(\left[\varkappa_{i, s} \vec{\nu}_{i}\right](r, t)-\lambda_{i} \vec{x}_{i, s}(r, t)\right) & =\overrightarrow{0}, \\
\varkappa_{i}(r, t) & =\bar{\varkappa}_{i}, \quad i=1 \rightarrow N,
\end{aligned}
$$

for $r \in\{0,1\}$ is an $L^{2}$-gradient flow of $E_{\lambda, \bar{\varkappa}}(\Gamma, \varkappa)$.

Proof. The desired results follow from (2.6a), (2.6b) and (2.8a), (2.8b), if we can show that the boundary terms in $(2.8 \mathrm{a}),(2.8 \mathrm{~b})$ vanish. In the case of $(2.8 \mathrm{a})$ this immediately follows from $(2.12 \mathrm{~b}),(2.12 \mathrm{c})$, while for $(2.8 \mathrm{~b})$ it follows from $(2.13 \mathrm{~b})$, (2.13c).

Remark 2.1. We remark that the boundary conditions in Theorem 2.1 are consistent with the fact that the flows $(2.6 \mathrm{a}),(2.6 \mathrm{~b})$ are fourth order in $\vec{x}$. In order to see this, there are various ways of counting the conditions. One approach is to view each curve $\Gamma_{i}(t)$ locally as a graph in the neighbourhood of a junction, so that $\vec{x}_{i}(\rho, t) \equiv\left(q(\rho), \vec{g}_{i}(q(\rho), t)\right)$, where $\vec{g}_{i}(\cdot, \cdot) \in \mathbb{R}^{d-1}$. Then the number of conditions required are as follows. On each curve we solve a fourth order parabolic system for $\vec{g}_{i}$, so we need $2(d-1)$ boundary conditions for each curve at a junction, and a condition to pin down $q(0) \in \mathbb{R}$; so we require $2 N(d-1)+1$ conditions in total. For this graph formulation, the attachment condition (2.12a) yields $(N-1)(d-1)$ conditions, the flux condition (2.12b) yields $d$, and the curvature condition (2.12c) yields $N(d-1)$, on recalling that $\vec{\varkappa}_{i}$ is always normal to $\Gamma_{i}$; and so $(2.12 \mathrm{a})-(2.12 \mathrm{c})$ yield $2 N(d-1)+1$ conditions in total at each junction.

An alternative counting procedure is to allow for tangential movement at the endpoint of each curve; that is, $\vec{x}_{i}(\rho, t) \equiv\left(q_{i}(\rho), \vec{g}_{i}\left(q_{i}(\rho), t\right)\right)$. This requires an extra $(N-1)$ conditions; as $q_{i}, i=1 \rightarrow N$, and not just $q$ have to be pinned down, which leads to $N(2 d-1)$ conditions in total. Under this counting procedure, the attachment condition $(2.12 \mathrm{a})$ now yields $(N-1) d$ conditions, an extra $(N-1)$ compared to the original graph count. Of course these counting procedures apply to $(2.13 \mathrm{a})-(2.13 \mathrm{c})$ with $d=2$. From now on, we will adopt the latter counting procedure throughout this paper. 
Of course the above counting procedure does not ensure that an initial value problem for (2.6a), (2.12a)-(2.12c) or (2.6b), (2.13a)-(2.13c) is well-posed. In order to show well-posedness the Lopatinski-Shapiro conditions for the linearized problem have to be considered. These conditions roughly state that the boundary conditions are independent "enough", we refer to Ref. 14 for a well-posedness result of another fourth order geometric flow with junctions. We expect that similar methods can be used for the above problems.

In the case of the $C^{1}$ junction conditions $(2.4 \mathrm{~b})$ the corresponding equation count is more subtle. In particular, deriving sufficiently weak conditions that make the last terms in (2.8a) and (2.8b) vanish is nontrivial. In order to achieve this, we define the following subsets of $\left(\mathbb{R}^{d}\right)^{N}$, which depend on $\Gamma, r \in\{0,1\}$ and $t \geq 0$. Let

$$
\begin{aligned}
\mathcal{N}_{\Gamma}(r, t) & :=\left\{\vec{a} \in\left(\mathbb{R}^{d}\right)^{N}: \vec{a}_{i} \cdot \vec{x}_{i, s}(r, t)=0, i=1 \rightarrow N\right\} \\
\text { and } \quad \Sigma_{\overrightarrow{0}}(r, t) & :=\left\{\vec{a} \in \mathcal{N}_{\Gamma}(r, t): \sum_{i=1}^{N} \vec{a}_{i}=\overrightarrow{0}\right\} .
\end{aligned}
$$

Theorem 2.2. Let the assumptions of Lemma 2.2 hold and let $\sum_{i=1}^{N} \vec{x}_{i, s}(r, 0)=\overrightarrow{0}$ for $r \in\{0,1\}$. Then the flow (2.6a), with the $C^{1}$ junction conditions

$$
\begin{array}{rlrl}
\vec{x}_{i}(r, t) & =\vec{x}_{1}(r, t), \quad i=2 \rightarrow N, \\
\sum_{i=1}^{N} \varsigma_{i}\left(\vec{\nabla}_{s} \vec{\varkappa}_{i}(r, t)+\frac{1}{2}\left(\left|\vec{\varkappa}_{i}\right|^{2}-2 \lambda_{i}\right) \vec{x}_{i, s}(r, t)\right) & =\overrightarrow{0}, & \\
\sum_{i=1}^{N} \vec{x}_{i, s}(r, t) & =\overrightarrow{0}, & \\
\sum_{i=1}^{N} \varsigma_{i} \vec{\varkappa}_{i}(r, t) \cdot \vec{a}_{i} & =0 & &
\end{array}
$$

for $r \in\{0,1\}$ is an $L^{2}$-gradient flow of $E_{\lambda}(\Gamma, \vec{\varkappa})$. Similarly, in the case $d=2$, the flow (2.6b) together with the $C^{1}$ junction conditions

$$
\begin{aligned}
\vec{x}_{i}(r, t)=\vec{x}_{1}(r, t) & \quad i=2 \rightarrow N, \\
\sum_{i=1}^{N} \varsigma_{i}\left(\left[\varkappa_{i, s} \vec{\nu}_{i}\right](r, t)+\frac{1}{2}\left(\varkappa_{i}^{2}-\bar{\varkappa}_{i}^{2}-2 \lambda_{i}\right) \vec{x}_{i, s}(r, t)\right) & =\overrightarrow{0}, \\
\sum_{i=1}^{N} \vec{x}_{i, s}(r, t) & =\overrightarrow{0}, \\
\sum_{i=1}^{N} \varsigma_{i}\left(\varkappa_{i}(r, t)-\bar{\varkappa}_{i}\right) \vec{\nu}_{i}(r, t) \cdot \vec{a}_{i} & =0 \quad \forall \vec{a} \in \Sigma_{\overrightarrow{0}}(r, t),
\end{aligned}
$$

for $r \in\{0,1\}$ is an $L^{2}$-gradient flow of $E_{\lambda, \bar{\varkappa}}(\Gamma, \varkappa)$.

Proof. Similarly to the proof of Theorem 2.1, we need to show that the boundary terms in (2.8a), (2.8b) vanish. In the case of (2.8a) this immediately follows from 
(2.15b)-(2.15d), while for $(2.8 \mathrm{~b})$ it follows from $(2.16 \mathrm{~b})-(2.16 \mathrm{~d})$, in both cases on noting that $\left(\vec{x}_{s, t}\right)(r, t) \in \Sigma_{\overrightarrow{0}}(r, t)$ for $r \in\{0,1\}$ can be chosen arbitrarily.

Remark 2.2. In view of Remark 2.1, in order to motivate that the number of conditions enforced in Theorem 2.2 is appropriate for the flow under consideration, it is sufficient to demonstrate that $(2.15 \mathrm{c}),(2.15 \mathrm{~d})$ enforce $N(d-1)$ conditions, i.e. the same number as $(2.12 \mathrm{c})$, and similarly that $(2.16 \mathrm{c}),(2.16 \mathrm{~d})$ enforce $N$ conditions. But on noting that $(2.12 \mathrm{c})$ can equivalently be formulated as

$$
\sum_{i=1}^{N} \varsigma_{i} \vec{\varkappa}_{i}(r, t) \cdot \vec{a}_{i}=0 \quad \forall \vec{a} \in \mathcal{N}_{\Gamma}(r, t) ;
$$

and on noting that $(2.15 \mathrm{c})$ is equivalent to $\left(\vec{x}_{s, t}\right)(r, t) \in \Sigma_{\overrightarrow{0}}(r, t)$ for $r \in\{0,1\}$, it follows immediately that $(2.15 \mathrm{c}),(2.15 \mathrm{~d})$ enforce the same number of conditions as (2.12c). The argument for $(2.16 \mathrm{c}),(2.16 \mathrm{~d})$ is analogous, on noting that $(2.13 \mathrm{c})$ for $d=2$ is equivalent to

$$
\sum_{i=1}^{N} \varsigma_{i}\left(\varkappa_{i}(r, t)-\bar{\varkappa}_{i}\right) \vec{\nu}_{i}(r, t) \cdot \vec{a}_{i}=0 \quad \forall \vec{a} \in \mathcal{N}_{\Gamma}(r, t) .
$$

Here we observe for the reader that $(2.15 \mathrm{c})$ and $(2.16 \mathrm{c})$, respectively, yield $d-1$ independent conditions if $N=2$ and $d$ conditions otherwise.

For some values of $N$ we now interpret the conditions $(2.15 \mathrm{a})-(2.15 \mathrm{~d})$ and (2.16a)-(2.16d) in more detail, where particular emphasis is put on $(2.15 \mathrm{~d})$ and (2.16d), respectively.

Corollary 2.1. Let the assumptions of Theorem 2.2 hold, and let $N=2$. Then the flow (2.6a), with the $C^{1}$ junction conditions

$$
\begin{aligned}
\vec{x}_{1}(r, t) & =\vec{x}_{2}(r, t), \\
\sum_{i=1}^{2} \varsigma_{i} \vec{\nabla}_{s} \vec{\varkappa}_{i}(r, t) & =\overrightarrow{0}, \\
\varsigma_{1}\left(\left|\vec{\varkappa}_{1}(r, t)\right|^{2}-2 \lambda_{1}\right) & =\varsigma_{2}\left(\left|\vec{\varkappa}_{2}(r, t)\right|^{2}-2 \lambda_{2}\right), \\
\sum_{i=1}^{2} \vec{x}_{i, s}(r, t) & =\overrightarrow{0}, \\
\varsigma_{1} \vec{\varkappa}_{1}(r, t) & =\varsigma_{2} \vec{\varkappa}_{2}(r, t),
\end{aligned}
$$

for $r \in\{0,1\}$ is an $L^{2}$-gradient flow of $E_{\lambda}(\Gamma, \vec{\varkappa})$. Similarly, in the case $d=2$, the 
flow (2.6b) together with the $C^{1}$ junction conditions

$$
\begin{aligned}
\vec{x}_{1}(r, t) & =\vec{x}_{2}(r, t), \\
\varsigma_{1} \varkappa_{1, s}(r, t) & =\varsigma_{2} \varkappa_{2, s}(r, t), \\
\varsigma_{1}\left(\varkappa_{1}^{2}(r, t)-\bar{\varkappa}_{1}^{2}-2 \lambda_{1}\right) & =\varsigma_{2}\left(\varkappa_{2}^{2}(r, t)-\bar{\varkappa}_{2}^{2}-2 \lambda_{2}\right), \\
\sum_{i=1}^{2} \vec{x}_{i, s}(r, t) & =\overrightarrow{0}, \\
\varsigma_{1}\left(\varkappa_{1}(r, t)-\bar{\varkappa}_{1}\right) & =-\varsigma_{2}\left(\varkappa_{2}(r, t)-\bar{\varkappa}_{2}\right),
\end{aligned}
$$

for $r \in\{0,1\}$ is an $L^{2}$-gradient flow of $E_{\lambda, \bar{\varkappa}}(\Gamma, \varkappa)$.

Proof. The conditions (2.17a)-(2.17e) are clearly equivalent to (2.15a)-(2.15d) for $N=2$. Similarly, for $d=2$ the conditions (2.18a)-(2.18e) are clearly equivalent to (2.16a)-(2.16d) for $N=2$ on noting that $\vec{\nu}_{1}=-\vec{\nu}_{2}$ at the two junctions.

Remark 2.3. It is not immediately clear whether the conditions $(2.17 \mathrm{a})-(2.17 \mathrm{e})$ and (2.18a)-(2.18e), respectively, always allow for a (unique) solution. For (2.17a)$(2.17 \mathrm{e})$ it is easy to see that when $\varsigma_{1}=\varsigma_{2}$ and $\lambda_{1}=\lambda_{2}$, then any pair $\left(\vec{\varkappa}_{1}, \vec{\varkappa}_{2}\right)$ with $\vec{\varkappa}_{1}=\vec{\varkappa}_{2}$ is a solution to $(2.17 \mathrm{c}),(2.17 \mathrm{e})$. We note that in the case $d=2$ this leads to $\varkappa_{1}=-\varkappa_{2}$. If $\varsigma_{1}=\varsigma_{2}$ and $\lambda_{1} \neq \lambda_{2}$, on the other hand, then there are no solutions that satisfy $(2.17 \mathrm{c}),(2.17 \mathrm{e})$. Lastly, if $\varsigma_{1} \neq \varsigma_{2}$ then any pair $\left(\vec{\varkappa}_{1}, \vec{\varkappa}_{2}\right)$ with $\varsigma_{1} \vec{\varkappa}_{1}=\varsigma_{2} \vec{\varkappa}_{2}$ and $\left|\vec{\varkappa}_{1}\right|^{2}=\frac{2 \varsigma_{2}\left(\varsigma_{1} \lambda_{1}-\varsigma_{2} \lambda_{2}\right)}{\varsigma_{1}\left(\varsigma_{2}-\varsigma_{1}\right)}$ is a solution. Clearly, the existence of such solutions will depend on the sign of $\left(\varsigma_{2}-\varsigma_{1}\right)\left(\varsigma_{1} \lambda_{1}-\varsigma_{2} \lambda_{2}\right)$.

We now turn our attention to (2.18c), (2.18e) and first consider the case $\varsigma_{1}=\varsigma_{2}$. Then there exists a unique solution $\left(\varkappa_{1}, \varkappa_{2}\right)$ to the two equations (2.18c), (2.18e) if $\bar{\varkappa}_{1}+\bar{\varkappa}_{2} \neq 0$. This unique solution is such that

$$
\left(\varkappa_{1}, \varkappa_{2}\right)=\left(\bar{\varkappa}_{1}+\frac{\lambda_{1}-\lambda_{2}}{\bar{\varkappa}_{1}+\bar{\varkappa}_{2}}, \bar{\varkappa}_{2}+\frac{\lambda_{2}-\lambda_{1}}{\bar{\varkappa}_{1}+\bar{\varkappa}_{2}}\right) .
$$

If $\bar{\varkappa}_{1}+\bar{\varkappa}_{2}=0$ then any pair $\left(\varkappa_{1}, \varkappa_{2}\right)$ with $\varkappa_{1}+\varkappa_{2}=0$ is a solution to $(2.18 \mathrm{c})$, (2.18e), provided that $\lambda_{1}=\lambda_{2}$. An example where this situation occurs is that of an expanding circle for, say, $\bar{\varkappa}_{1}=\bar{\varkappa}_{2}=0$. If $\bar{\varkappa}_{1}+\bar{\varkappa}_{2}=0$ and $\lambda_{1} \neq \lambda_{2}$ then no solution exists.

The case $\varsigma_{1} \neq \varsigma_{2}$ is slightly more involved. If $\varsigma_{1} \lambda_{1}=\varsigma_{2} \lambda_{2}$, then the two solutions $\left(\varkappa_{1}, \varkappa_{2}\right)=\left(\bar{\varkappa}_{1}, \bar{\varkappa}_{2}\right)$ and

$$
\left(\varkappa_{1}, \varkappa_{2}\right)=\left(\frac{\left(\varsigma_{1}+\varsigma_{2}\right) \bar{\varkappa}_{1}+2 \varsigma_{2} \bar{\varkappa}_{2}}{\varsigma_{1}-\varsigma_{2}}, \frac{\left(\varsigma_{1}+\varsigma_{2}\right) \bar{\varkappa}_{2}+2 \varsigma_{1} \bar{\varkappa}_{1}}{\varsigma_{2}-\varsigma_{1}}\right)
$$

exist, with the two solutions being distinct if and only if $\bar{\varkappa}_{1}+\bar{\varkappa}_{2} \neq 0$. Otherwise the sign of the term $\varsigma_{1} \varsigma_{2}\left(\bar{\varkappa}_{1}+\bar{\varkappa}_{2}\right)^{2}+2\left(\varsigma_{2}-\varsigma_{1}\right)\left(\varsigma_{1} \lambda_{1}-\varsigma_{2} \lambda_{2}\right)$ determines whether there are no solutions $(<0)$, a unique solution $(=0)$ or exactly two solutions $(>0)$.

Remark 2.4. In applications it is often natural to look at elastic flows with constraints on the lengths of the curves. A time-dependent $\lambda(t) \in \mathbb{R}^{N}$ in (2.6a), (2.6b) 
can then also be interpreted as a Lagrange multiplier for side constraints on $\left|\Gamma_{i}(t)\right|$, $i=1 \rightarrow N$. We will avoid technical difficulties arising from the possibly degenerate situations discussed e.g. for $N=2$ in Remark 2.3, by introducing $\lambda(t) \in \mathbb{R}^{N}$ in the weak setting below as a free parameter in a generalized Lagrangian. See Remark 2.9 for more details.

Remark 2.5. It turns out that the conditions (2.17a)-(2.17e) and (2.18a)-(2.18e) yield that the two curves meet at a $C^{2}$ junction, if certain compatibility conditions are met. The conditions are that $\varsigma_{1}=\varsigma_{2}$ and, if $d=2, \bar{\varkappa}_{1}+\bar{\varkappa}_{2}=0$. It then immediately follows from Remark 2.3 that also $\lambda_{1}=\lambda_{2}$ in order for the junction conditions to make sense. Hence the two curves $\Gamma_{1}$ and $\Gamma_{2}$ have identical energy densities. In other words, the energies (2.2) and (2.3) do not "see" the junction points at all. It is then not surprising, that the two junctions are of type $C^{2}$. To see this, we note that $(2.17 \mathrm{e})$ implies $\vec{x}_{1, s s}=\vec{x}_{2, s s}$ at the two junction points if $\varsigma_{1}=\varsigma_{2}$. Similarly, in the case $d=2$ it follows from $(2.18 \mathrm{e})$ that $\varkappa_{1}=-\varkappa_{2}$ if $\varsigma_{1}=\varsigma_{2}$ and $\bar{\varkappa}_{1}+\bar{\varkappa}_{2}=0$. Together with $(2.18 \mathrm{~d})$ this yields that $\vec{x}_{1, s s}=\varkappa_{1} \vec{\nu}_{1}=-\varkappa_{2} \vec{\nu}_{1}=$ $\varkappa_{2} \vec{\nu}_{2}=\vec{x}_{2, s s}$ at the two junctions, so that again the junctions are in fact of type $C^{2}$.

Corollary 2.2. Let the assumptions of Theorem 2.2 hold and let $N=3$. First let $d=3$. Then the flow (2.6a), with the $C^{1}$ junction conditions (2.15a)-(2.15c) and

$$
\begin{gathered}
\varsigma_{1} \vec{\varkappa}_{1}(r, t) \cdot \overrightarrow{\mathrm{n}}(r, t)=\varsigma_{2} \vec{\varkappa}_{2}(r, t) \cdot \overrightarrow{\mathrm{n}}(r, t)=\varsigma_{3} \vec{\varkappa}_{3}(r, t) \cdot \overrightarrow{\mathrm{n}}(r, t), \\
\sum_{i=1}^{3} \varsigma_{i}\left[\vec{\varkappa}_{i} \cdot \vec{x}_{i, s}^{\perp}\right](r, t)=0
\end{gathered}
$$

for $r \in\{0,1\}$ is an $L^{2}$-gradient flow of $E_{\lambda}(\Gamma, \vec{\varkappa})$. Here ${ }^{\perp}$ denotes a rotation through $\frac{\pi}{2}$ in the two-dimensional plane spanned by $\left\{\vec{x}_{1, s}(r, t), \vec{x}_{2, s}(r, t), \vec{x}_{3, s}(r, t)\right\}$, with unit normal $\overrightarrow{\mathrm{n}}(r, t)$. Similarly, in the case $d=2$, the flow (2.6b) together with the $C^{1}$ junction conditions (2.16a)-(2.16c) and

$$
\sum_{i=1}^{3} \varsigma_{i}\left(\varkappa_{i}(r, t)-\bar{\varkappa}_{i}\right)=0,
$$

for $r \in\{0,1\}$ is an $L^{2}$-gradient flow of $E_{\lambda, \bar{\varkappa}}(\Gamma, \varkappa)$.

Proof. It is sufficient to show that $(2.15 \mathrm{~d})$ for $N=3$ is equivalent to $(2.19 \mathrm{a})$, $(2.19 \mathrm{~b})$ if $(2.15 \mathrm{a})-(2.15 \mathrm{c})$ hold. But this follows immediately from the fact that the three vectors $\left(\vec{x}_{1, s}^{\perp}, \vec{x}_{2, s}^{\perp}, \vec{x}_{3, s}^{\perp}\right)(r, t),(\vec{n},-\overrightarrow{\mathrm{n}}, \overrightarrow{0})(r, t)$ and $(\overrightarrow{\mathrm{n}}, \overrightarrow{0},-\overrightarrow{\mathrm{n}})(r, t)$ form a basis for $\Sigma_{\overrightarrow{0}}(r, t)$ in $\left(\mathbb{R}^{3}\right)^{3}$. Similarly, for $d=2$ it is sufficient to show that $(2.16 \mathrm{~d})$ for $N=3$ is equivalent to $(2.20)$, if $(2.16 \mathrm{a})-(2.16 \mathrm{c})$ hold. But this follows immediately from the fact that $\vec{a}=\vec{\nu}(r, t)$ is a basis for $\Sigma_{\overrightarrow{0}}(r, t)$ in $\left(\mathbb{R}^{2}\right)^{3}$.

Remark 2.6. In Corollaries 2.1 and 2.2 we only considered the cases $N=2$ and $N=3$ in detail. This can be generalized to larger values of $N$, however the interpretation of the meaning of $(2.15 \mathrm{~d})$ and $(2.16 \mathrm{~d})$, respectively, then becomes more 
complicated. In particular, analogously to the proof of Corollary 2.2, we can rewrite (2.16d) equivalently as

$$
\sum_{i=1}^{N} \varsigma_{i}\left(\varkappa_{i}(r, t)-\bar{\varkappa}_{i}\right) \alpha_{i}=0 \quad \forall \alpha \in\left\{\beta \in \mathbb{R}^{N}: \sum_{i=1}^{N} \beta_{i} \vec{\nu}_{i}(r, t)=\overrightarrow{0}\right\} .
$$

The conditions enforced by (2.21) can be made more explicit by selecting a basis for the set defined there. For instance, $(2.18 \mathrm{e})$ corresponds to $\alpha=(1,1),(2.20)$ corresponds to $\alpha=(1,1,1)$, while $N=4$ would correspond to e.g. $\alpha_{1}=(1,0,1,0)$ and $\alpha_{2}=(0,1,0,1)$, and $N=6$ yields e.g. $\alpha_{1}=(1,0,0,1,0,0), \alpha_{2}=(0,1,0,0,1,0)$, $\alpha_{3}=(0,0,1,0,0,1)$ and $\alpha_{4}=(1,0,1,0,1,0)$.

Theorem 2.3. Let the assumptions of Lemma 2.2 hold and let $\sum_{i=1}^{N} \vec{x}_{i, s}(r, 0)=\overrightarrow{0}$ for $r \in\{0,1\}$. Let $\vec{\alpha}_{0}, \vec{\alpha}_{1} \in \mathbb{R}^{d}$. Then the flow (2.6a), with the $C^{1}$ spline node conditions

$$
\begin{aligned}
\vec{x}_{i}(r, t) & =\vec{\alpha}_{r}, \quad i=1 \rightarrow N, \\
\sum_{i=1}^{N} \vec{x}_{i, s}(r, t) & =\overrightarrow{0}, \\
\sum_{i=1}^{N} \varsigma_{i} \vec{\varkappa}_{i}(r, t) \cdot \vec{a}_{i} & =0 \quad \forall \vec{a} \in \Sigma_{\overrightarrow{0}}(r, t),
\end{aligned}
$$

for $r \in\{0,1\}$ is an $L^{2}$-gradient flow of $E_{\lambda}(\Gamma, \overrightarrow{\mathcal{\varkappa}})$. Similarly, in the case $d=2$, the flow (2.6b) together with the $C^{1}$ spline node conditions

$$
\begin{aligned}
\vec{x}_{i}(r, t) & =\vec{\alpha}_{r}, \quad i=1 \rightarrow N, \\
\sum_{i=1}^{N} \vec{x}_{i, s}(r, t) & =\overrightarrow{0}, \\
\sum_{i=1}^{N} \varsigma_{i}\left(\varkappa_{i}(r, t)-\bar{\varkappa}_{i}\right) \vec{\nu}_{i}(r, t) \cdot \vec{a}_{i} & =0 \quad \forall \vec{a} \in \Sigma_{\overrightarrow{0}}(r, t),
\end{aligned}
$$

for $r \in\{0,1\}$ is an $L^{2}$-gradient flow of $E_{\lambda, \bar{\varkappa}}(\Gamma, \varkappa)$.

Proof. Similarly to the proof of Theorem 2.1, we need to show that the boundary terms in $(2.8 \mathrm{a}),(2.8 \mathrm{~b})$ vanish. It follows from (2.22a) and (2.23a) that (2.8a), (2.8b) hold with $\vec{x}_{1, t}=\overrightarrow{0}$. The remaining boundary term in (2.8a) vanishes on noting (2.22b), (2.22c). Similarly, in the case $d=2$ it follows from (2.23b), (2.23c) that the remaining boundary term in $(2.8 \mathrm{~b})$ vanishes.

For the following corollary we assume that $N=2$ and that the two curves are oriented in such a way that $\vec{x}_{1}(0, t)=\vec{x}_{2}(1, t)$ and $\vec{x}_{1}(1, t)=\vec{x}_{2}(0, t)$. This will be the natural situation for $C^{1}$ spline junctions adopted from now on throughout the paper. 
Corollary 2.3. Let $N=2$ and let $\vec{x}(\rho, t):[0,1] \times[0, T] \rightarrow\left(\mathbb{R}^{d}\right)^{2}$ be a parameterization of the curve network $\Gamma(t)=\vec{x}([0,1], t)$ with $\vec{x}_{1}(r, 0)=\vec{x}_{2}(1-r, 0)=\vec{\alpha}_{r}$ and $\vec{x}_{1, s}(r, 0)=\vec{x}_{2, s}(1-r, 0)$ for $r \in\{0,1\}$, where $\vec{\alpha}_{0}, \vec{\alpha}_{1} \in \mathbb{R}^{d}$. Let $\lambda, \varkappa \in \mathbb{R}^{2}, \varsigma \in \mathbb{R}_{>0}^{2}$. Then the flow (2.6a), with the $C^{1}$ spline node conditions

$$
\begin{aligned}
\vec{x}_{1}(r, t) & =\vec{x}_{2}(1-r, t)=\vec{\alpha}_{r}, \\
\vec{x}_{1, s}(r, t) & =\vec{x}_{2, s}(1-r, t), \\
\varsigma_{1} \vec{\varkappa}_{1}(r, t) & =\varsigma_{2} \vec{\varkappa}_{2}(1-r, t),
\end{aligned}
$$

for $r \in\{0,1\}$ is an $L^{2}$-gradient flow of $E_{\lambda}(\Gamma, \vec{\varkappa})$. Similarly, in the case $d=2$, the flow (2.6b) together with the $C^{1}$ spline node conditions

$$
\begin{aligned}
\vec{x}_{1}(r, t) & =\vec{x}_{2}(1-r, t)=\vec{\alpha}_{r}, \\
\vec{x}_{1, s}(r, t) & =\vec{x}_{2, s}(1-r, t), \\
\varsigma_{1}\left(\varkappa_{1}(r, t)-\bar{\varkappa}_{1}\right) & =\varsigma_{2}\left(\varkappa_{2}(1-r, t)-\bar{\varkappa}_{2}\right),
\end{aligned}
$$

for $r \in\{0,1\}$ is an $L^{2}$-gradient flow of $E_{\lambda, \bar{\varkappa}}(\Gamma, \varkappa)$.

Proof. The conditions (2.24a)-(2.24c) are clearly equivalent to (2.22a)-(2.22c) for $N=2$, on noting the different orientation of $\Gamma_{2}$ assumed here. Similarly, for $d=2$ the conditions $(2.25 \mathrm{a})-(2.25 \mathrm{c})$ are clearly equivalent to $(2.23 \mathrm{a})-(2.23 \mathrm{c})$ for $N=2$ on noting that $\vec{\nu}_{1}=\vec{\nu}_{2}$ at the two junctions for the orientation assumed here.

Remark 2.7. In Corollary 2.3, and in Theorem 2.3, in order to keep the presentation in the above theorems uniform, we included in the case $d=2$ non-zero values of $\varkappa$ for the smooth spline junctions. In practice, however, this is not of particular interest. Therefore we will usually consider $\bar{\varkappa}=0$ in the spline junction case from now on.

Remark 2.8. Similarly to Remark 2.5, we see that (2.24c) and (2.25c) lead to a $C^{2}$-spline junction if $\varsigma_{1}=\varsigma_{2}$ and if $\varsigma_{1}=\varsigma_{2}, \bar{\varkappa}_{1}=\bar{\varkappa}_{2}$, respectively. This follows from the fact that $(2.24 \mathrm{c})$ yields that $\vec{x}_{1, s s}=\vec{x}_{2, s s}$, while it follows from $(2.25 \mathrm{c})$ and (2.25b) that $\vec{x}_{1, s s}=\varkappa_{1} \vec{\nu}_{1}=\varkappa_{1} \vec{\nu}_{2}=\varkappa_{2} \vec{\nu}_{2}=\vec{x}_{2, s s}$ at the two junctions.

For later use, we introduce the definitions

$$
\begin{aligned}
\underline{V} & :=\left\{\vec{\chi} \in\left[H^{1}\left((0,1), \mathbb{R}^{d}\right)\right]^{N}: \vec{\chi}_{1}=\vec{\chi}_{2}=\ldots=\vec{\chi}_{N} \text { on }\{0,1\}\right\} \\
\text { and } \quad \underline{W} & :=\left[H_{0}^{1}\left((0,1), \mathbb{R}^{d}\right)\right]^{N}
\end{aligned}
$$

and similarly $V \subset\left[H^{1}((0,1), \mathbb{R})\right]^{N}, W=\left[H_{0}^{1}((0,1), \mathbb{R})\right]^{N}$ and $U=\left[L^{2}((0,1), \mathbb{R})\right]^{N}$. In addition, for arbitrary $\chi, \eta \in U$ we define

$$
\langle\chi, \eta\rangle_{\Gamma}:=\sum_{i=1}^{N}\left\langle\chi_{i}, \eta_{i}\right\rangle_{\Gamma_{i}},
$$

and similarly for vector- and matrix-valued functions. 


\subsection{The $C^{0}$ case - attachment only}

We now consider a weak formulation of the $L^{2}$-gradient flow of the energy (2.2) together with (2.4a). Hence the strong formulation of the flow is given by (2.6a) with $(2.12 \mathrm{a})-(2.12 \mathrm{c})$.

We will use the formal calculus of PDE constrained optimization in order to formally derive the $L^{2}$-gradient flow of $E_{\lambda}(\Gamma, \vec{\varkappa})$ for $\vec{x} \in \underline{V}$ and $\vec{\varkappa} \in \underline{W}$ under the side conditions

$$
\begin{aligned}
\langle\vec{\varkappa}, \vec{\eta}\rangle_{\Gamma}+\left\langle\vec{x}_{s}, \vec{\eta}_{s}\right\rangle_{\Gamma} & =0 & \forall \vec{\eta} \in \underline{W} \\
\text { and } \quad & \left\langle\vec{\varkappa} \cdot \vec{x}_{s}, \chi\right\rangle_{\Gamma}=0 & \forall \chi \in U .
\end{aligned}
$$

Although the derivation is formal the resulting equations will fulfill a stability inequality, which will also hold on the semidiscrete level. We observe that (2.28a) is the natural weak formulation of (2.1) given that only (2.12a)-(2.12c) holds at the two junction points. The side constraint (2.28b) trivially holds, however we include it here to mimic the procedure on the discrete level, where its inclusion leads to equidistributed spatial discretizations. We now introduce the Lagrange multipliers $\vec{y} \in \underline{W}$ and $z \in U$ for (2.28a), (2.28b), and define the Lagrangian

$$
\mathcal{L}(\vec{x}, \vec{\varkappa}, \vec{y}, z):=\frac{1}{2}\langle\varsigma \vec{\varkappa}, \vec{\varkappa}\rangle_{\Gamma}+\langle\varsigma, \lambda\rangle_{\Gamma}-\langle\vec{\varkappa}, \vec{y}\rangle_{\Gamma}-\left\langle\vec{x}_{s}, \vec{y}_{s}\right\rangle_{\Gamma}+\left\langle\vec{\varkappa} . \vec{x}_{s}, z\right\rangle_{\Gamma} .
$$

Hence we obtain, on taking variations $\left[\frac{\delta}{\delta \vec{x}} \mathcal{L}\right](\vec{\chi}),\left[\frac{\delta}{\delta \vec{\varkappa}} \mathcal{L}\right](\vec{\xi}),\left[\frac{\delta}{\delta \vec{y}} \mathcal{L}\right](\vec{\eta})$ and $\left[\frac{\delta}{\delta z} \mathcal{L}\right](\chi)$, that the direction of steepest descent of $E_{\lambda}$ under the constraints (2.28a), (2.28b) is given by $-\left[\frac{\delta}{\delta \vec{x}} \mathcal{L}\right](\vec{\chi})$, with the remaining variations of $\mathcal{L}$ set to zero. In particular, we obtain the gradient flow

$$
\begin{aligned}
& \left\langle\vec{P} \vec{x}_{t}, \vec{\chi}\right\rangle_{\Gamma}=\left\langle\vec{\nabla}_{s} \vec{y}, \overrightarrow{\nabla_{s}} \vec{\chi}\right\rangle_{\Gamma}-\frac{1}{2}\left\langle\left(\varsigma|\vec{\varkappa}|^{2}-2 \vec{\varkappa} \cdot \vec{y}+2 \varsigma \lambda\right) \vec{x}_{s}, \vec{\chi}_{s}\right\rangle_{\Gamma}-\left\langle z \vec{\varkappa}, \vec{\chi}_{s}\right\rangle_{\Gamma} \\
& \forall \vec{\chi} \in \underline{V},(2.30 \mathrm{a}) \\
& \left\langle\varsigma \vec{\varkappa}+z \vec{x}_{s}-\vec{y}, \vec{\xi}\right\rangle_{\Gamma}=0 \quad \forall \vec{\xi} \in \underline{W}, \\
& \langle\vec{\varkappa}, \vec{\eta}\rangle_{\Gamma}+\left\langle\vec{x}_{s}, \vec{\eta}_{s}\right\rangle_{\Gamma}=0 \quad \forall \vec{\eta} \in \underline{W}, \\
& \left\langle\vec{x} \cdot \vec{x}_{s}, \chi\right\rangle_{\Gamma}=0 \quad \forall \chi \in U .
\end{aligned}
$$

It follows from $(2.30 \mathrm{~b}),(2.30 \mathrm{~d})$ that

$$
\vec{P}_{i} \vec{y}_{i}=\varsigma_{i} \vec{\varkappa}_{i} \quad \text { and } \quad z_{i}=\vec{y}_{i} \cdot \vec{x}_{i, s}, \quad i=1 \rightarrow N .
$$

Hence the normal part of the Lagrange multiplier $\vec{y}$ agrees with the weighted curvature vector $\varsigma \vec{\varkappa}$, but in addition it may have a nonzero tangential component $z$. Overall our formal weak formulation of the $L^{2}$-gradient flow for (2.2) subject to $(2.28 \mathrm{a}),(2.28 \mathrm{~b})$ can now be formulated as: Given $\Gamma(0)$, for all $t \in(0, T]$ find $\Gamma(t)=\vec{x}([0,1], t)$, with $\vec{x}(t) \in \underline{V}$, and $\vec{y}(t) \in \underline{W}$ such that

$$
\begin{aligned}
& \left\langle\vec{P} \vec{x}_{t}, \vec{\chi}\right\rangle_{\Gamma}-\left\langle\vec{\nabla} \vec{y}_{s} \vec{y}, \vec{\nabla}_{s} \vec{\chi}\right\rangle_{\Gamma}-\frac{1}{2}\left\langle\left(\varsigma^{-1}|\vec{P} \vec{y}|^{2}-2 \varsigma \lambda\right) \vec{x}_{s}, \vec{\chi}_{s}\right\rangle_{\Gamma}+\left\langle\varsigma^{-1}\left(\vec{y} \cdot \vec{x}_{s}\right) \vec{P} \vec{y}, \vec{\chi}_{s}\right\rangle_{\Gamma} \\
& =0 \quad \forall \vec{\chi} \in \underline{V},(2.32 \mathrm{a}) \\
& \left\langle\varsigma^{-1} \vec{P} \vec{y}, \vec{\eta}\right\rangle_{\Gamma}+\left\langle\vec{x}_{s}, \vec{\eta}_{s}\right\rangle_{\Gamma}=0 \quad \forall \vec{\eta} \in \underline{W} .
\end{aligned}
$$


On recalling (2.31) we observe that in the above weak formulation of (2.6a) with $(2.12 \mathrm{a})-(2.12 \mathrm{c})$, the conditions $(2.12 \mathrm{a}),(2.12 \mathrm{c})$ are enforced strongly, while $(2.12 \mathrm{~b})$ is enforced weakly through $(2.32 \mathrm{a})$.

In the case $d=2$, we consider the $L^{2}$-gradient flow of the energy (2.3) together with (2.4a). Hence the strong formulation of the flow is given by (2.6b) with (2.13a)(2.13c).

Similarly to the higher codimension case, we formally derive the $L^{2}$-gradient flow of $E_{\lambda, \bar{\varkappa}}(\Gamma, \varkappa)$ for $\vec{x} \in \underline{V}$ and $(\varkappa-\bar{\varkappa}) \in W$ under the side condition

$$
\langle\varkappa \vec{\nu}, \vec{\eta}\rangle_{\Gamma}+\left\langle\vec{x}_{s}, \vec{\eta}_{s}\right\rangle_{\Gamma}=0 \quad \forall \vec{\eta} \in \underline{W} .
$$

As before, we define the Lagrangian

$$
\mathcal{L}(\vec{x}, \varkappa, \vec{y}):=\frac{1}{2}\left\langle\varsigma,(\varkappa-\bar{\varkappa})^{2}\right\rangle_{\Gamma}+\langle\varsigma, \lambda\rangle_{\Gamma}-\langle\varkappa \vec{\nu}, \vec{y}\rangle_{\Gamma}-\left\langle\vec{x}_{s}, \vec{y}_{s}\right\rangle_{\Gamma},
$$

where $\vec{y}(t) \in \underline{W}$ is a Lagrange multiplier for the side constraint (2.33). Hence we obtain, on taking variations $\left[\frac{\delta}{\delta \vec{x}} \mathcal{L}\right](\vec{\chi}),\left[\frac{\delta}{\delta \varkappa} \mathcal{L}\right](\chi)$ and $\left[\frac{\delta}{\delta \vec{y}} \mathcal{L}\right](\vec{\eta})$, and on setting $\left\langle\vec{P} \vec{x}_{t}, \vec{\chi}\right\rangle_{\Gamma}=-\left[\frac{\delta}{\delta \vec{x}} \mathcal{L}\right](\vec{\chi})$, that

$$
\begin{aligned}
& \left\langle\vec{P} \vec{x}_{t}, \vec{\chi}\right\rangle_{\Gamma}=\left\langle\vec{\nabla}_{s} \vec{y}, \vec{\nabla}_{s} \vec{\chi}\right\rangle_{\Gamma}-\frac{1}{2}\left\langle\left[\varsigma(\varkappa-\bar{\varkappa})^{2}+2 \varsigma \lambda-2 \varkappa(\vec{y} \cdot \vec{\nu})\right] \vec{x}_{s}, \vec{\chi}_{s}\right\rangle_{\Gamma} \\
& -\left\langle\varkappa \vec{y},\left(\vec{\nabla}_{s} \vec{\chi}\right)^{\perp}\right\rangle_{\Gamma} \quad \forall \vec{\chi} \in \underline{V}, \\
& \langle\varsigma(\varkappa-\bar{\varkappa})-\vec{y} \cdot \vec{\nu}, \chi\rangle_{\Gamma}=0 \quad \forall \chi \in W, \\
& \langle\varkappa \vec{\nu}, \vec{\eta}\rangle_{\Gamma}+\left\langle\vec{x}_{s}, \vec{\eta}_{s}\right\rangle_{\Gamma}=0 \quad \forall \vec{\eta} \in \underline{W} .
\end{aligned}
$$

Clearly, it follows from $(2.35 \mathrm{~b}),(\varkappa-\bar{\varkappa}) \in W$ and $\vec{y} \in \underline{W}$ that

$$
\varsigma_{i}\left(\varkappa_{i}-\bar{\varkappa}_{i}\right)=\vec{y}_{i} \cdot \vec{\nu}_{i}, \quad i=1 \rightarrow N \text {. }
$$

Our weak formulation of this gradient flow is then given by: Given $\Gamma(0)$, for all $t \in(0, T]$ find $\Gamma(t)=\vec{x}([0,1], t)$, with $\vec{x}(t) \in \underline{V}$, and $\vec{y}(t) \in \underline{W}$ such that

$$
\begin{gathered}
\left\langle\vec{P} \vec{x}_{t}, \vec{\chi}\right\rangle_{\Gamma}-\left\langle\vec{\nabla}_{s} \vec{y}, \vec{\nabla}_{s} \vec{\chi}\right\rangle_{\Gamma}+\frac{1}{2}\left\langle\left[\varsigma^{-1}(\vec{y} \cdot \vec{\nu})^{2}+2 \varsigma \lambda\right] \vec{x}_{s}, \vec{\chi}_{s}\right\rangle_{\Gamma} \\
-\left\langle\left(\varsigma^{-1} \vec{y} \cdot \vec{\nu}+\bar{\varkappa}\right) \vec{y}^{\perp}, \vec{\chi}_{s}\right\rangle_{\Gamma}=0 \quad \forall \vec{\chi} \in \underline{V}, \\
\left\langle\varsigma^{-1} \vec{P} \vec{y}, \vec{\eta}\right\rangle_{\Gamma}+\left\langle\vec{x}_{s}, \vec{\eta}_{s}\right\rangle_{\Gamma}=-\langle\bar{\varkappa} \vec{\nu}, \vec{\eta}\rangle_{\Gamma} \quad \forall \vec{\eta} \in \underline{W} .
\end{gathered}
$$

On recalling (2.36) we observe that in the above weak formulation of (2.6b) with (2.13a)-(2.13c), the conditions (2.13a), (2.13c) are enforced strongly, while (2.13b) is enforced weakly through (2.37a).

On adapting the techniques in Ref. 7, it is now straightforward to derive stability theorems for $(2.32 \mathrm{a}),(2.32 \mathrm{~b})$ and $(2.37 \mathrm{a}),(2.37 \mathrm{~b})$. In particular, the following theorem shows that $(2.32 \mathrm{a}),(2.32 \mathrm{~b})$ and $(2.37 \mathrm{a}),(2.37 \mathrm{~b})$ formulate $L^{2}$-gradient flows for the energies (2.2) and (2.3), respectively.

Theorem 2.4. Let $(\vec{x}(t), \vec{y}(t))_{t \in(0, T]}$ be a solution to (2.32a), (2.32b). Then we have that

$$
\frac{\mathrm{d}}{\mathrm{d} t} E_{\lambda}\left(\Gamma, \varsigma^{-1} \vec{P} \vec{y}\right)=-\left\langle\vec{P} \vec{x}_{t}, \vec{P} \vec{x}_{t}\right\rangle_{\Gamma} \leq 0
$$


where $\varsigma^{-1} \vec{P} \vec{y}=\vec{\varkappa}$ are the curvature vectors on $\Gamma$. Moreover, if $d=2$ and $(\vec{x}(t), \vec{y}(t))_{t \in(0, T]}$ is a solution to (2.37a), (2.37b), then it holds that

$$
\frac{\mathrm{d}}{\mathrm{d} t} E_{\lambda, \bar{\varkappa}}\left(\Gamma, \varsigma^{-1} \vec{y} \cdot \vec{\nu}+\bar{\varkappa}\right)=-\left\langle\vec{P} \vec{x}_{t}, \vec{P} \vec{x}_{t}\right\rangle_{\Gamma} \leq 0,
$$

where $\varsigma^{-1} \vec{y} \cdot \vec{\nu}+\bar{\varkappa}=\varkappa$ are the curvatures on $\Gamma$.

Proof. Differentiating (2.32b) with respect to $t$ yields, on noting (2.10), that

$$
\left\langle\varsigma^{-1}(\vec{P} \vec{y})_{t}, \vec{\eta}\right\rangle_{\Gamma}+\left\langle\varsigma^{-1}(\vec{P} \vec{y}) \cdot \vec{\eta}, \vec{x}_{s} \cdot \vec{x}_{t, s}\right\rangle_{\Gamma}+\left\langle\vec{\nabla}_{s} \vec{x}_{t}, \vec{\nabla}_{s} \vec{\eta}\right\rangle_{\Gamma}=0 \quad \forall \vec{\eta} \in \underline{W} .
$$

On choosing $\vec{\eta}=\vec{y} \in \underline{W}$ in (2.39), we obtain that

$$
\left\langle\varsigma^{-1}(\vec{P} \vec{y})_{t}, \vec{y}\right\rangle_{\Gamma}+\left\langle\varsigma^{-1}|\vec{P} \vec{y}|^{2}, \vec{x}_{s}, \vec{x}_{t, s}\right\rangle_{\Gamma}+\left\langle\vec{\nabla}_{s} \vec{x}_{t}, \vec{\nabla}_{s} \vec{y}\right\rangle_{\Gamma}=0 .
$$

Combining (2.40) and (2.32a) with $\vec{\chi}=\vec{x}_{t} \in \underline{V}$ yields that

$$
\begin{array}{r}
\left\langle\varsigma^{-1}(\vec{P} \vec{y})_{t}, \vec{y}\right\rangle_{\Gamma}+\frac{1}{2}\left\langle\varsigma^{-1}|\vec{P} \vec{y}|^{2}+2 \varsigma \lambda, \vec{x}_{s} \cdot \vec{x}_{t, s}\right\rangle_{\Gamma}+\left\langle\varsigma^{-1}\left(\vec{y} \cdot \vec{x}_{s}\right) \vec{P} \vec{y}, \vec{x}_{t, s}\right\rangle_{\Gamma} \\
=-\left\langle\vec{P} \vec{x}_{t}, \vec{P} \vec{x}_{t}\right\rangle_{\Gamma} .
\end{array}
$$

The desired result (2.38a) then follows from (2.41) and (2.31), on noting that

$$
\begin{aligned}
\left\langle(\vec{P} \vec{y})_{t},(\overrightarrow{I d}-\vec{P}) \vec{y}\right\rangle_{\Gamma} & =\left\langle(\vec{P} \vec{y})_{t},\left(\vec{y} \cdot \vec{x}_{s}\right) \vec{x}_{s}\right\rangle_{\Gamma}=-\left\langle\vec{P} \vec{y},\left(\vec{y} \cdot \vec{x}_{s}\right)\left(\vec{x}_{s}\right)_{t}\right\rangle_{\Gamma} \\
& =-\left\langle\left(\vec{y} \cdot \vec{x}_{s}\right) \vec{P} \vec{y}, \vec{x}_{t, s}\right\rangle_{\Gamma} .
\end{aligned}
$$

The proof of (2.38b) is similar. Differentiating (2.37b) with respect to $t$ and then choosing $\vec{\eta}=\vec{y} \in \underline{W}$ yields that

$$
\begin{aligned}
\left\langle\varsigma^{-1}(\vec{P} \vec{y})_{t}, \vec{y}\right\rangle_{\Gamma}+\left\langle\varsigma^{-1}(\vec{y} \cdot \vec{\nu})^{2}, \vec{x}_{s} \cdot \vec{x}_{t, s}\right\rangle_{\Gamma}+\left\langle\vec{\nabla}_{s} \vec{x}_{t}, \vec{\nabla}_{s} \vec{y}\right\rangle_{\Gamma} & \\
& =-\left\langle\vec{\nu}_{t}+\left(\vec{x}_{s} \cdot \vec{x}_{t, s}\right) \vec{\nu}, \bar{\varkappa} \vec{y}\right\rangle_{\Gamma} .
\end{aligned}
$$

Combining (2.42) and (2.37a) with $\vec{\chi}=\vec{x}_{t} \in \underline{V}$ yields that

$$
\begin{aligned}
\left\langle\varsigma^{-1}(\vec{P} \vec{y})_{t}, \vec{y}\right\rangle_{\Gamma}+\frac{1}{2}\left\langle 3 \varsigma^{-1}(\vec{y} \cdot \vec{\nu})^{2}+2 \varsigma \lambda, \vec{x}_{s} \cdot \vec{x}_{t, s}\right\rangle_{\Gamma}-\left\langle\varsigma^{-1}(\vec{y} \cdot \vec{\nu}) \vec{y}^{\perp}, \vec{x}_{t, s}\right\rangle_{\Gamma} & +\left\langle\vec{P} \vec{x}_{t}, \vec{P} \vec{x}_{t}\right\rangle_{\Gamma} \\
= & -\left\langle\left(\vec{x}_{t, s}\right)^{\perp}+\vec{\nu}_{t}+\left(\vec{x}_{s} \cdot \vec{x}_{t, s}\right) \vec{\nu}, \vec{\varkappa} \vec{y}\right\rangle_{\Gamma} .
\end{aligned}
$$

Noting that $\vec{\nu}_{i, t}=-\left(\vec{\nabla}_{s} \vec{x}_{i, t}\right)^{\perp}, i=1 \rightarrow N$, and that

$$
\vec{y}_{i}^{\perp}=\left(\vec{y}_{i} \cdot \vec{\nu}_{i}\right) \vec{x}_{i, s}-\left(\vec{y}_{i} \cdot \vec{x}_{i, s}\right) \vec{\nu}_{i}, \quad i=1 \rightarrow N,
$$

yields that (2.43) collapses to

$$
\begin{array}{r}
\left\langle\varsigma^{-1}(\vec{P} \vec{y})_{t}, \vec{y}\right\rangle_{\Gamma}+\frac{1}{2}\left\langle\varsigma^{-1}(\vec{y} \cdot \vec{\nu})^{2}+2 \varsigma \lambda, \vec{x}_{s} \cdot \vec{x}_{t, s}\right\rangle_{\Gamma}+\left\langle\varsigma^{-1}\left(\vec{y} \cdot \vec{x}_{s}\right) \vec{P} \vec{y}, \vec{x}_{t, s}\right\rangle_{\Gamma} \\
=-\left\langle\vec{P} \vec{x}_{t}, \vec{P} \vec{x}_{t}\right\rangle_{\Gamma} .
\end{array}
$$

The desired result (2.38b) then follows from (2.45) on noting (2.10) and (2.36). 


\subsection{The $C^{1}$ case - enforcing Young's law}

In this subsection we first consider a weak formulation of the $L^{2}$-gradient flow of the energy (2.2) together with (2.4b). Hence the strong formulation of the flow is given by $(2.6 \mathrm{a})$ with $(2.15 \mathrm{a})-(2.15 \mathrm{~d})$.

In order to avoid technical difficulties, we pursue a slightly different approach to $\S 2.1$ here. In particular, in view of (2.31), we immediately introduce an auxiliary function $\vec{u} \in \underline{V}$ such that

$$
\vec{P}_{i} \vec{u}_{i}=\varsigma_{i} \vec{\varkappa}_{i}, \quad i=1 \rightarrow N
$$

Clearly, $\vec{u} \in \underline{V}$ together with (2.46) enforces the curvature junction conditions (2.15d). Taking variations with respect to $\vec{u}$ is now straightforward, whereas working directly with $\vec{\varkappa}$ would be highly nontrivial. The existence of a function $\vec{u} \in \underline{V}$ satisfying (2.46) can be argued as follows. If $N \geq 3$, then (2.46) means that $d$ linearly independent equations need to hold at the junctions, recall Remark 2.2, which agrees with the degrees of freedom in $\vec{u}$ at the junctions. If $N=2$, on the other hand, the $C^{1}$ conditions mean that (2.46) only yields $d-1$ linearly independent equations. Thus we could enforce the additional constraint

$$
\vec{u}_{i}(r, t)=\varsigma_{i} \vec{\varkappa}_{i}(r, t), \quad r \in\{0,1\}, \quad t \in[0, T], \quad i=1 \rightarrow 2,
$$

in order to have the same number of constraints as unknowns. Note that (2.47) collapses to only one additional condition, since $\vec{u}_{2}(r, t)=\vec{u}_{1}(r, t)$ from $\vec{u} \in \underline{V}$, and $\varsigma_{1} \vec{\varkappa}_{1}(r, t)=\varsigma_{2} \vec{\varkappa}_{2}(r, t)$ from $(2.17 \mathrm{e})$. A similar approach to $(2.47)$ will be used on the discrete level later on.

We consider the $L^{2}$-gradient flow of $E_{\lambda}\left(\Gamma, \varsigma^{-1} \vec{P} \vec{u}\right)$ for $\vec{x} \in \underline{V}$ and $\vec{u} \in \underline{V}$ under the side condition

$$
\left\langle\varsigma^{-1} \vec{P} \vec{u}, \vec{\eta}\right\rangle_{\Gamma}+\left\langle\vec{x}_{s}, \vec{\eta}_{s}\right\rangle_{\Gamma}=0 \quad \forall \vec{\eta} \in \underline{V}
$$

We observe that $(2.48)$ is the natural weak formulation of (2.1) in view of $(2.15 \mathrm{c})$ and (2.46).

We introduce the Lagrange multiplier $\vec{y} \in \underline{V}$ for (2.48), and define the Lagrangian

$$
\mathcal{L}(\vec{x}, \vec{u}, \vec{y}):=\frac{1}{2}\left\langle\varsigma^{-1},|\vec{P} \vec{u}|^{2}\right\rangle_{\Gamma}+\langle\varsigma, \lambda\rangle_{\Gamma}-\left\langle\varsigma^{-1} \vec{P} \vec{u}, \vec{y}\right\rangle_{\Gamma}-\left\langle\vec{x}_{s}, \vec{y}_{s}\right\rangle_{\Gamma}
$$

Hence we obtain, on taking variations $\left[\frac{\delta}{\delta \vec{x}} \mathcal{L}\right](\vec{\chi}),\left[\frac{\delta}{\delta \vec{u}} \mathcal{L}\right](\vec{\xi})$ and $\left[\frac{\delta}{\delta \vec{y}} \mathcal{L}\right](\vec{\eta})$, that the direction of steepest descent of $E_{\lambda}$ under the constraint $(2.48)$ is given by $-\left[\frac{\delta}{\delta \vec{x}} \mathcal{L}\right](\vec{\chi})$, with the remaining variations of $\mathcal{L}$ set to zero. In particular, we obtain the gradient 
flow

$$
\begin{aligned}
& \left\langle\vec{P} \vec{x}_{t}, \vec{\chi}\right\rangle_{\Gamma}=\left\langle\vec{\nabla}_{s} \vec{y}, \vec{\nabla}_{s} \vec{\chi}\right\rangle_{\Gamma}-\frac{1}{2}\left\langle\left(\varsigma^{-1}|\vec{P} \vec{u}|^{2}-2 \varsigma^{-1} \vec{P} \vec{u} \cdot \vec{y}+2 \varsigma \lambda\right) \vec{x}_{s}, \vec{\chi}_{s}\right\rangle_{\Gamma} \\
& +\left\langle\varsigma^{-1} \vec{P} \vec{u},\left(\vec{u} \cdot \vec{x}_{s}\right) \vec{\chi}_{s}\right\rangle_{\Gamma}-\left\langle\varsigma^{-1} \vec{y},\left(\vec{u} \cdot \vec{x}_{s}\right) \vec{P} \vec{\chi}_{s}+\left(\vec{P} \vec{u} \cdot \vec{\chi}_{s}\right) \vec{x}_{s}\right\rangle_{\Gamma} \\
& \forall \vec{\chi} \in \underline{V}, \\
& \left\langle\varsigma^{-1} \vec{P} \vec{u}, \vec{\xi}\right\rangle_{\Gamma}-\left\langle\varsigma^{-1} \vec{y}, \vec{P} \vec{\xi}\right\rangle_{\Gamma}=0 \quad \forall \vec{\xi} \in \underline{V}, \\
& \left\langle\varsigma^{-1} \vec{P} \vec{u}, \vec{\eta}\right\rangle_{\Gamma}+\left\langle\vec{x}_{s}, \vec{\eta}_{s}\right\rangle_{\Gamma}=0 \quad \forall \vec{\eta} \in \underline{V},
\end{aligned}
$$

where we have noted that

$$
\left[\frac{\delta}{\delta \vec{x}} \vec{P} \vec{u}\right](\vec{\chi})=-\left(\vec{u} \cdot \vec{x}_{s}\right) \vec{P} \vec{\chi}_{s}-\left(\vec{u} \cdot \vec{P} \vec{\chi}_{s}\right) \vec{x}_{s}
$$

It follows from (2.50b) and (2.46) that

$$
\vec{P}_{i} \vec{y}_{i}=\vec{P}_{i} \vec{u}_{i}=\varsigma_{i} \vec{\varkappa}_{i}, \quad i=1 \rightarrow N .
$$

Similarly to $§ 2.1$, it is now possible to eliminate $\vec{u}$ from $(2.50 \mathrm{a}),(2.50 \mathrm{c})$ in order to obtain the following formal weak formulation: Given $\Gamma(0)$, for all $t \in(0, T]$ find $\Gamma(t)=\vec{x}([0,1], t)$, with $\vec{x}(t) \in \underline{V}$, and $\vec{y}(t) \in \underline{V}$ such that

$$
\begin{array}{rr}
\left\langle\vec{P} \vec{x}_{t}, \vec{\chi}\right\rangle_{\Gamma}-\left\langle\vec{\nabla}_{s} \vec{y}, \vec{\nabla}_{s} \vec{\chi}\right\rangle_{\Gamma}-\frac{1}{2}\left\langle\left(\varsigma^{-1}|\vec{P} \vec{y}|^{2}-2 \varsigma \lambda\right) \vec{x}_{s}, \vec{\chi}_{s}\right\rangle_{\Gamma}+\left\langle\varsigma^{-1}\left(\vec{y} \cdot \vec{x}_{s}\right) \vec{P} \vec{y}, \vec{\chi}_{s}\right\rangle_{\Gamma} \\
=0 \quad \forall \vec{\chi} \in \underline{V}, & \\
\left\langle\varsigma^{-1} \vec{P} \vec{y}, \vec{\eta}\right\rangle_{\Gamma}+\left\langle\vec{x}_{s}, \vec{\eta}_{s}\right\rangle_{\Gamma}=0 & \forall \vec{\eta} \in \underline{V} .53 \mathrm{a})
\end{array}
$$

On recalling (2.31) we observe that in the above weak formulation of (2.6a) with (2.15a)-(2.15c), the conditions (2.15a) are enforced strongly, while (2.15b), (2.15c) are enforced weakly through (2.53a), (2.53b), respectively. Moreover, the conditions (2.15d) are enforced strongly via $\vec{y} \in \underline{V}$ on recalling (2.52).

In the case $d=2$, the flow for the energy (2.3) subject to the $C^{1}$ junction conditions $(2.4 \mathrm{~b})$ can be treated analogously. In particular, and similarly to (2.46), we introduce $\vec{u} \in \underline{V}$ such that

$$
\vec{P}_{i} \vec{u}_{i}=\varsigma_{i}\left(\varkappa_{i}-\bar{\varkappa}_{i}\right) \vec{\nu}_{i}, \quad i=1 \rightarrow N
$$

Similarly to $(2.47)$, in the case $N=2$ we could enforce the additional condition that

$$
\vec{u}_{i}(r, t)=\varsigma_{i}\left(\varkappa_{i}(r, t)-\bar{\varkappa}_{i}\right) \vec{\nu}_{i}(r, t), \quad r \in\{0,1\}, \quad t \in[0, T], \quad i=1 \rightarrow 2 .
$$

We then consider the $L^{2}$-gradient flow of $E_{\lambda, \bar{\varkappa}}\left(\Gamma, \varsigma^{-1} \vec{u} \cdot \vec{\nu}+\bar{\varkappa}\right)$ for $\vec{x} \in \underline{V}$ and $\vec{u} \in \underline{V}$ under the side condition

$$
\left\langle\varsigma^{-1} \vec{P} \vec{u}, \vec{\eta}\right\rangle_{\Gamma}+\left\langle\vec{x}_{s}, \vec{\eta}_{s}\right\rangle_{\Gamma}=-\langle\bar{\varkappa} \vec{\nu} \vec{\eta}\rangle_{\Gamma} \quad \forall \vec{\eta} \in \underline{V} .
$$

We observe that (2.56) is the natural weak formulation of (2.1) in view of (2.16c) and (2.54). 
On introducing the Lagrange multiplier $\vec{y} \in \underline{V}$ for (2.54), the Lagrangian is now given by $(2.49)$ with the additional term $-\langle\bar{\varkappa} \vec{\nu}, \vec{y}\rangle_{\Gamma}$. Hence, on taking variations, we obtain $(2.50 \mathrm{a})-(2.50 \mathrm{c})$, with the additional terms

$$
\left\langle\bar{\varkappa}(\vec{y} \cdot \vec{\nu}) \vec{x}_{s}, \vec{\chi}_{s}\right\rangle_{\Gamma}-\left\langle\bar{\varkappa} \vec{y},\left(\vec{\nabla}_{s} \vec{\chi}\right)^{\perp}\right\rangle_{\Gamma}
$$

on the right hand side of $(2.50 \mathrm{a})$, and with $-\langle\bar{\varkappa} \vec{\nu}, \vec{\eta}\rangle_{\Gamma}$ on the right hand side of (2.50c). In particular, we obtain once again that $\vec{P} \vec{y}=\vec{P} \vec{u}$.

Overall, our weak formulation of this gradient flow is then given by: Given $\Gamma(0)$, for all $t \in(0, T]$ find $\Gamma(t)=\vec{x}([0,1], t)$, with $\vec{x}(t) \in \underline{V}$, and $\vec{y}(t) \in \underline{V}$ such that

$$
\begin{gathered}
\left\langle\vec{P} \vec{x}_{t}, \vec{\chi}\right\rangle_{\Gamma}-\left\langle\vec{\nabla}_{s} \vec{y}, \vec{\nabla}_{s} \vec{\chi}\right\rangle_{\Gamma}+\frac{1}{2}\left\langle\left[\varsigma^{-1}(\vec{y} \cdot \vec{\nu})^{2}+2 \varsigma \lambda\right] \vec{x}_{s}, \vec{\chi}_{s}\right\rangle_{\Gamma} \\
-\left\langle\left(\varsigma^{-1} \vec{y} \cdot \vec{\nu}+\bar{\varkappa}\right) \vec{y}^{\perp}, \vec{\chi}_{s}\right\rangle_{\Gamma}=0 \quad \forall \vec{\chi} \in \underline{V}, \\
\left\langle\varsigma^{-1} \vec{P} \vec{y}, \vec{\eta}\right\rangle_{\Gamma}+\left\langle\vec{x}_{s}, \vec{\eta}_{s}\right\rangle_{\Gamma}=-\langle\vec{\varkappa} \vec{\nu}, \vec{\eta}\rangle_{\Gamma} \quad \forall \vec{\eta} \in \underline{V} .
\end{gathered}
$$

Here

$$
\varsigma_{i}\left(\varkappa_{i}-\bar{\varkappa}_{i}\right)=\vec{y}_{i} \cdot \vec{\nu}_{i}, \quad i=1 \rightarrow N
$$

As before, we observe that in the above weak formulation of (2.6b) with (2.16a)(2.16d), the conditions (2.16a) are enforced strongly, while (2.16b), (2.16c) are enforced weakly through $(2.58 \mathrm{a}),(2.58 \mathrm{~b})$, respectively. In addition, the conditions (2.16d) are enforced strongly through $\vec{y} \in \underline{V}$ on noting (2.59).

Similarly to Theorem 2.4, the following theorem holds.

Theorem 2.5. Let $(\vec{x}(t), \vec{y}(t))_{t \in(0, T]}$ be a solution to (2.53a), (2.53b). Then we have that

$$
\frac{\mathrm{d}}{\mathrm{d} t} E_{\lambda}\left(\Gamma, \varsigma^{-1} \vec{P} \vec{y}\right)=-\left\langle\vec{P} \vec{x}_{t}, \vec{P} \vec{x}_{t}\right\rangle_{\Gamma} \leq 0,
$$

where $\varsigma^{-1} \vec{P} \vec{y}=\vec{\varkappa}$ are the curvature vectors on $\Gamma$. Moreover, if $d=2$ and $(\vec{x}(t), \vec{y}(t))_{t \in(0, T]}$ is a solution to (2.58a), (2.58b) then it holds that

$$
\frac{\mathrm{d}}{\mathrm{d} t} E_{\lambda, \bar{\varkappa}}\left(\Gamma, \varsigma^{-1} \vec{y} \cdot \vec{\nu}+\bar{\varkappa}\right)=-\left\langle\vec{P} \vec{x}_{t}, \vec{P} \vec{x}_{t}\right\rangle_{\Gamma} \leq 0,
$$

where $\varsigma^{-1} \vec{y} \cdot \vec{\nu}+\bar{\varkappa}=\varkappa$ are the curvatures on $\Gamma$.

Proof. The proof is exactly the same as the proof of Theorem 2.4, with only the change from $\underline{W}$ to $\underline{V}$ for the trial space of $\vec{y}$ and for the test space for $(2.53 \mathrm{~b})$ and $(2.58 \mathrm{~b})$.

Remark 2.9. In order to handle length constraints for any of the flows considered so far, we adopt the following formal procedure. In the definitions of the Lagrangians we replace the term $\langle\varsigma, \lambda\rangle_{\Gamma}$ by

$$
\sum_{i=1}^{N} \varsigma_{i} \lambda_{i}\left(\left|\Gamma_{i}\right|-l_{i}\right),
$$


where $l \in \mathbb{R}_{>0}^{N}$ are given lengths, and we treat $\lambda(t) \in \mathbb{R}^{N}$ as a free variable. Here $\left|\Gamma_{i}\right|:=\langle 1,1\rangle_{\Gamma_{i}}$. Now taking the variation of this modified Lagrangian with respect to $\lambda$ gives the additional equations

$$
\left|\Gamma_{i}\right|=l_{i}, \quad i=1 \rightarrow N .
$$

For example, as the length preserving variant of the elastic flow in the case of the $C^{1}$ junction conditions (2.4b) we then obtain (2.53a), (2.53b) with the additional unknown $\lambda(t)$ and the additional constraints (2.61) for $l_{i}:=\left|\Gamma_{i}(0)\right|, i=1 \rightarrow N$. Similarly to Theorem 2.5 , it is a simple matter to show that this length preserving flow fulfills $\frac{\mathrm{d}}{\mathrm{d} t} E_{0}\left(\Gamma, \varsigma^{-1} \vec{P} \vec{y}\right)=-\left\langle\vec{P} \vec{x}_{t}, \vec{P} \vec{x}_{t}\right\rangle_{\Gamma} \leq 0$.

\subsection{Nonlinear $C^{2}$ splines}

In this subsection, we consider the elastic flow for the junction conditions (2.4c) for $N=2$, and here in particular the setting in Corollary 2.3. Given the results of the two previous subsections, it is straightforward to derive the appropriate weak formulation of this flow, namely e.g. (2.32a), (2.32b) with the roles of $\underline{W}$ and $\underline{V}$ exactly reversed, so that $\vec{x}_{t} \in \underline{W}$ and $\vec{y} \in \underline{V}$. We note that of practical interest is usually the case $\varsigma_{1}=\varsigma_{2}$ with, in addition, $\bar{\varkappa}_{1}=\bar{\varkappa}_{2}=0$ if $d=2$. As mentioned earlier, recall Remark 2.8, the junction conditions (2.4c) for the flow of (2.2) then immediately yield that the junction is actually of type $C^{2}$. Hence we refer to curves obtained in this way as nonlinear $C^{2}$ splines. Of course, if $\varsigma_{1} \neq \varsigma_{2}$ or $\bar{\varkappa}_{1} \neq \bar{\varkappa}_{2}$, then the junctions will be only $C^{1}$ in general.

Once the strong conditions and the weak formulations are known for the simple setup considered in Corollary 2.3, i.e. two curves meeting at two spline node junctions, it is not difficult to generalize the results to more than two spline nodes, and to possibly open curve chains as depicted in Figure 1. We will do this in more detail in the remainder of this subsection.

Given a set of points $\left\{\vec{\alpha}_{j}\right\}_{j=0}^{N}$, the aim is to find a $C^{1}$ curve that interpolates these points such that the curvature energy becomes minimal. In order to differentiate the total spline curve that is sought from the curve segments that make up the chain, we will refer to the former curve as a "chain" from now on. The chain can either be open or closed, recall Figure 1. The closed chain case is a very small deviation of the situations studied so far, and so from now on we will only consider the open chain case. This means that there are $N$ curves, meeting at the $N-1$ double junctions $\left\{\vec{\alpha}_{j}\right\}_{j=1}^{N-1}$, together with appropriate boundary conditions at $\vec{\alpha}_{0}$ and $\vec{\alpha}_{N}$.

A natural way to obtain such nonlinear splines as minimizers for the curvature energy is to consider a gradient flow for the energy (2.2) subject to the desired junction and boundary conditions.

We remark that the special case $N=1$, i.e. a single open curve with prescribed boundary conditions at the two endpoints, has been considered by the authors in Ref. 7. Physically relevant boundary conditions are so-called clamped conditions, where position and angle(s) are fixed, and so-called Navier conditions, where posi- 
tion and curvature is fixed. Once again, to simplify the presentation we will only consider the case of clamped boundary conditions from now on. The details of how to incorporate Navier boundary conditions can be found in Ref. 7.

We introduce the following test and trial spaces for our formulation. Let

$$
\begin{aligned}
\underline{V}_{s} & :=\left\{\vec{\chi} \in\left[H^{1}\left((0,1), \mathbb{R}^{d}\right)\right]^{N}: \vec{\chi}_{i}(1)=\vec{\chi}_{i+1}(0), \quad i=1 \rightarrow N-1\right\}, \\
\underline{W}_{s} & :=\left\{\vec{\chi} \in \underline{V}_{s}: \vec{\chi}_{1}(0)=\vec{\chi}_{N}(1)=\overrightarrow{0}\right\}
\end{aligned}
$$

and similarly $V_{s}, W_{s} \subset\left[H^{1}((0,1), \mathbb{R})\right]^{N}$; and we also recall the space $\underline{W}$ from $(2.26 \mathrm{~b})$.

For completeness, we state the strong formulation of the flow that we consider.

Theorem 2.6. Let $\vec{x}(\rho, t):[0,1] \times[0, T] \rightarrow\left(\mathbb{R}^{d}\right)^{N}$ be a parameterization of the curve network $\Gamma(t)=\vec{x}([0,1], t)$. Let $\lambda, \bar{\varkappa} \in \mathbb{R}^{N}, \varsigma \in \mathbb{R}_{>0}^{N}$ and $\vec{\alpha}_{l} \in \mathbb{R}^{d}, l=0 \rightarrow N$. Let $\vec{\zeta}_{0}, \vec{\zeta}_{N} \in \mathbb{S}^{d-1}:=\left\{\vec{p} \in \mathbb{R}^{d}:|\vec{p}|=1\right\}$. Then the flow (2.6a), with the $C^{1}$ spline node conditions

$$
\begin{aligned}
\vec{x}_{i}(1, t) & =\vec{x}_{i+1}(0, t)=\vec{\alpha}_{i} & & i=1 \rightarrow N-1, \\
\vec{x}_{i, s}(1, t) & =\vec{x}_{i+1, s}(0, t) & & i=1 \rightarrow N-1, \\
\varsigma_{i} \vec{\varkappa}_{i}(1, t) & =\varsigma_{i+1} \vec{\varkappa}_{i+1}(0, t), & & i=1 \rightarrow N-1,
\end{aligned}
$$

together with the clamped boundary conditions

$$
\text { (a) } \vec{x}_{1}(0, t)=\vec{\alpha}_{0}, \vec{x}_{N}(1, t)=\vec{\alpha}_{N} \quad \text { and } \quad(b) \vec{x}_{1, s}(0, t)=\vec{\zeta}_{0}, \vec{x}_{N, s}(1, t)=\vec{\zeta}_{N}
$$

is an $L^{2}$-gradient flow of $E_{\lambda}(\Gamma, \vec{\varkappa})$, provided that (2.63a), (2.63b) and (2.64) hold at time $t=0$. In addition, the flow (2.6a), with the $C^{1}$ spline node conditions (2.63a)-(2.63c) together with the homogeneous Navier boundary conditions

$$
\text { (a) } \vec{x}_{1}(0, t)=\vec{\alpha}_{0}, \vec{x}_{N}(1, t)=\vec{\alpha}_{N} \quad \text { and } \quad(b) \vec{\varkappa}_{1}(0, t)=\overrightarrow{0}, \vec{\varkappa}_{N}(1, t)=\overrightarrow{0}
$$

is also an $L^{2}$-gradient flow of $E_{\lambda}(\Gamma, \vec{\varkappa})$, provided that (2.63a), (2.63b) and (2.65) hold at time $t=0$. Similarly, in the case $d=2$, the flow (2.6b) together with the $C^{1}$ spline node conditions

$$
\begin{aligned}
\vec{x}_{i}(1, t) & =\vec{x}_{i+1}(0, t)=\vec{\alpha}_{i} & & i=1 \rightarrow N-1, \\
\vec{x}_{i, s}(1, t) & =\vec{x}_{i+1, s}(0, t) & & i=1 \rightarrow N-1, \\
\varsigma_{i}\left(\varkappa_{i}(1, t)-\varkappa_{i}\right) & =\varsigma_{i+1}\left(\varkappa_{i+1}(0, t)-\bar{\varkappa}_{i+1}\right) & & i=1 \rightarrow N-1,
\end{aligned}
$$

and the clamped boundary conditions (2.64) is an $L^{2}$-gradient flow of $E_{\lambda, \bar{\varkappa}}(\Gamma, \varkappa)$, provided that (2.66a), (2.66b) and (2.64) hold at time $t=0$.

Proof. The desired results follow from Corollary 2.3 and Lemma 2.1; see also Theorem 2.3 in Ref. 7.

We then have the following weak formulation of the flow (2.6a) with (2.63a)(2.63c) and (2.64). Given $\Gamma(0)=\vec{x}([0,1], 0)$ with $\vec{x}(0) \in \underline{V}_{s}$, for all $t \in(0, T]$ find 
$\Gamma(t)=\vec{x}([0,1], t)$, with $\vec{x}_{t}(t) \in \underline{W}$, and $\vec{y}(t) \in \underline{V}_{s}$ such that

$$
\begin{aligned}
& \left\langle\vec{P} \vec{x}_{t}, \vec{\chi}\right\rangle_{\Gamma}-\left\langle\vec{\nabla} \vec{y}, \overrightarrow{\nabla_{s}} \vec{\chi}\right\rangle_{\Gamma}-\frac{1}{2}\left\langle\left(\varsigma^{-1}|\vec{P} \vec{y}|^{2}-2 \varsigma \lambda\right) \vec{x}_{s}, \vec{\chi}_{s}\right\rangle_{\Gamma}+\left\langle\varsigma^{-1}\left(\vec{y} \cdot \vec{x}_{s}\right) \vec{P} \vec{y}, \vec{\chi}_{s}\right\rangle_{\Gamma} \\
& =0 \quad \forall \vec{\chi} \in \underline{W}, \quad(2.67 \mathrm{a}) \\
& \left\langle\varsigma^{-1} \vec{P} \vec{y}, \vec{\eta}\right\rangle_{\Gamma}+\left\langle\vec{x}_{s}, \vec{\eta}_{s}\right\rangle_{\Gamma}=\vec{\zeta}_{N} \cdot \vec{\eta}_{N}(1)-\vec{\zeta}_{0} \cdot \vec{\eta}_{1}(0) \quad \forall \vec{\eta} \in \underline{V}_{s} ;
\end{aligned}
$$

where, similarly to (2.31), $\vec{P}_{i} \vec{y}_{i}=\varsigma_{i} \vec{\varkappa}_{i}, i=1 \rightarrow N$. We observe that in the above weak formulation of (2.6a) with (2.63a)-(2.63c), (2.64) the conditions (2.63a), (2.64a) are enforced strongly, while $(2.63 \mathrm{~b}),(2.64 \mathrm{~b})$ are enforced weakly through (2.67a), (2.67b), respectively. Moreover, the conditions (2.63c) are enforced in a strong sense through $\vec{y} \in \underline{V}_{s}$.

Similarly, a weak formulation of the flow (2.6a) with (2.63a)-(2.63c) and (2.65) is given by $(2.67 \mathrm{a}),(2.67 \mathrm{~b})$ with a zero right hand side in $(2.67 \mathrm{~b})$ and with $\underline{V}_{s}$ replaced by $\underline{W}_{s}$. We omit the weak formulation of the flow (2.6b) with (2.66a)(2.66c) and (2.64). For nonzero $\bar{\varkappa}$ this flow is not of practical interest. For $\bar{\varkappa}=0$, on the other hand, it collapses to the flow (2.6a) with (2.63a)-(2.63c) and (2.64). Nevertheless, deriving the weak formulation and the resulting finite element schemes is straightforward.

\subsection{More general situations}

In the previous subsections we have discussed in detail the situation where $N$ curves meet at two junctions for different types of junctions, and for nonlinear splines we also looked at chains of curves, where chains of curves meet at an ordered sequence of double junctions. The above can be generalized to other situations. E.g. a chain of the type on the left of Figure 1, but with $C^{0}$ or $C^{1}$ double junctions in place of a $C^{1}$ spline junction. Equally, the situation in the middle of Figure 1 but now with a $C^{0}$ or $C^{1}$ junction would be possible. In each case, the corresponding weak formulations are straightforward and we do not present the details here. But we note that the finite element approximations introduced later on in this paper will easily carry across to these more general situations.

\section{Semidiscrete finite element approximation}

In this section we introduce continuous-in-time semidiscrete finite element approximations of the elastic flows with junctions discussed in Section 2. In particular, we will repeat on a discrete level the considerations in Section 2 and, as a consequence, we will derive spatially discrete finite element approximations that are stable and that fulfill an equidistribution property, similarly to the semidiscrete schemes considered in e.g. Ref. 7.

For $N \geq 1$, let $[0,1]=\cup_{j=1}^{J_{i}} I_{j}^{i}, i=1 \rightarrow N$, be decompositions of $[0,1]$ into intervals $I_{j}^{i}=\left[q_{j-1}^{i}, q_{j}^{i}\right]$ based on the nodes $\left\{q_{j}^{i}\right\}_{j=0}^{J_{i}}, J_{i} \geq 2$. Let $h_{j}^{i}=\left|I_{j}^{i}\right|$ and $h=$ $\max _{i=1 \rightarrow N} \max _{j=1 \rightarrow J_{i}} h_{j}^{i}$ be the maximal length of a grid element. The appropriate 
finite element spaces are then defined by

$$
\underline{V}^{h}:=\left\{\vec{\chi} \in \underline{V} \cap\left[C\left([0,1], \mathbb{R}^{d}\right)\right]^{N}:\left.\vec{\chi}_{i}\right|_{I_{j}^{i}} \text { is linear } \forall j=1 \rightarrow J_{i}, i=1 \rightarrow N\right\} \subset \underline{V}
$$

and similarly for the spaces $\underline{W}^{h} \subset \underline{W}, W^{h} \subset W$ and $\underline{V}_{s}^{h} \subset \underline{V}_{s}$. We define the unconstrained finite element spaces $\underline{S}^{h}:=\left\{\vec{\chi} \in\left[C\left([0,1], \mathbb{R}^{d}\right)\right]^{N}:\left.\vec{\chi}_{i}\right|_{I_{j}^{i}}\right.$ is linear $\forall j=$ $\left.1 \rightarrow J_{i}, \quad i=1 \rightarrow N\right\}$; and similarly $S^{h} \subset[C([0,1], \mathbb{R})]^{N}$. In addition, let $\pi^{h}:[C([0,1], \mathbb{R})]^{N} \rightarrow S^{h}$ be the standard Lagrange interpolation operator, and similarly for all the other finite element spaces, e.g. $\pi^{h}:\left[C\left([0,1], \mathbb{R}^{d}\right)\right]^{N} \rightarrow \underline{S}^{h}$.

In this section, we consider a family $\left(\Gamma^{h}(t)\right)_{t \in[0, T]}$ of curve networks parameterized by $\vec{X}^{h}(t) \in \underline{V}^{h}$. Here we make the natural assumption that

$\left(\mathcal{C}^{h}\right)$ Let $\vec{X}_{i}^{h}\left(q_{j}^{i}, t\right) \neq \vec{X}_{i}^{h}\left(q_{j+1}^{i}, t\right), j=0 \rightarrow J_{i}-1$, and $\vec{X}_{i}^{h}\left(q_{j-1}^{i}, t\right) \neq \vec{X}_{i}^{h}\left(q_{j+1}^{i}, t\right)$, $j=1 \rightarrow J_{i}-1$, for $i=1 \rightarrow N$ and for all $t \in[0, T]$.

The condition $\left(\mathcal{C}^{h}\right)$ ensures for every polygonal curve of the curve network $\Gamma^{h}(t)$ that each element has positive length and that no two neighbouring elements coalesce. In addition, we recall that $\langle u, v\rangle_{\Gamma^{h}}=\sum_{i=1}^{N}\left\langle u_{i}, v_{i}\right\rangle_{\Gamma_{i}^{h}}=\sum_{i=1}^{N} \int_{0}^{1} u_{i} \cdot v_{i}\left|\vec{X}_{i, \rho}^{h}\right| \mathrm{d} \rho$ and, if $u, v$ are piecewise continuous, with possible jumps at the nodes $\left\{q_{j}\right\}_{j=1}^{J}$, we define the mass lumped inner product

$$
\begin{aligned}
\langle u, v\rangle_{\Gamma^{h}}^{h} & :=\sum_{i=1}^{N}\left\langle u_{i}, v_{i}\right\rangle_{\Gamma_{i}^{h}}^{h} \\
& :=\sum_{i=1}^{N} \frac{1}{2} \sum_{j=1}^{J_{i}}\left|\vec{X}_{i}^{h}\left(q_{j}^{i}, t\right)-\vec{X}_{i}^{h}\left(q_{j-1}^{i}, t\right)\right|\left[(u . v)\left(\left(q_{j}^{i}\right)^{-}\right)+(u \cdot v)\left(\left(q_{j-1}^{i}\right)^{+}\right)\right]
\end{aligned}
$$

where we define $u\left(q^{ \pm}\right):=\lim _{\varepsilon \searrow 0} u(q \pm \varepsilon)$.

We introduce the following differential operators on $\Gamma^{h}$. Let $D_{s}, \widehat{D}_{s}: S^{h} \rightarrow S^{h}$ be such that $D_{s} \eta=\left(D_{s}^{1} \eta_{1}, \ldots, D_{s}^{N} \eta_{N}\right)$, where

$$
\begin{aligned}
& \left(D_{s}^{i} \eta_{i}\right)\left(q_{j}^{i}\right) \\
& \quad=\frac{\left|\vec{X}_{i}^{h}\left(q_{j}^{i}, t\right)-\vec{X}_{i}^{h}\left(q_{j-1}^{i}, t\right)\right| \eta_{i, s}\left(\left(q_{j}^{i}\right)^{-}\right)+\left|\vec{X}_{i}^{h}\left(q_{j+1}^{i}, t\right)-\vec{X}_{i}^{h}\left(q_{j}^{i}, t\right)\right| \eta_{i, s}\left(\left(q_{j}^{i}\right)^{+}\right)}{\left|\vec{X}_{i}^{h}\left(q_{j}^{i}, t\right)-\vec{X}_{i}^{h}\left(q_{j-1}^{i}, t\right)\right|+\left|\vec{X}_{i}^{h}\left(q_{j+1}^{i}, t\right)-\vec{X}_{i}^{h}\left(q_{j}^{i}, t\right)\right|} \\
& \quad=\frac{\eta_{i}\left(q_{j+1}^{i}\right)-\eta_{i}\left(q_{j-1}^{i}\right)}{\left|\vec{X}_{i}^{h}\left(q_{j}^{i}, t\right)-\vec{X}_{i}^{h}\left(q_{j-1}^{i}, t\right)\right|+\left|\vec{X}_{i}^{h}\left(q_{j+1}^{i}, t\right)-\vec{X}_{i}^{h}\left(q_{j}^{i}, t\right)\right|} \\
& \left(\widehat{D}_{s}^{i} \eta_{i}\right)\left(q_{j}^{i}\right)=\frac{\left(D_{s}^{i} \eta_{i}\right)\left(q_{j}^{i}\right)}{\left|\left(D_{s}^{i} \vec{X}_{i}^{h}(t)\right)\left(q_{j}^{i}\right)\right|}=\frac{\eta_{i}\left(q_{j+1}^{i}\right)-\eta_{i}\left(q_{j-1}^{i}\right)}{\left|\vec{X}_{i}^{h}\left(q_{j+1}^{i}, t\right)-\vec{X}_{i}^{h}\left(q_{j-1}^{i}, t\right)\right|}
\end{aligned}
$$

for $j=1 \rightarrow J_{i}-1$; and

$$
\left(D_{s}^{i} \eta_{i}\right)\left(q_{0}^{i}\right)=\left(\widehat{D}_{s}^{i} \eta_{i}\right)\left(q_{0}^{i}\right)=\left.\eta_{i, s}\right|_{I_{1}^{i}} \quad \text { and } \quad\left(D_{s}^{i} \eta_{i}\right)\left(q_{J_{i}}^{i}\right)=\left(\widehat{D}_{s}^{i} \eta_{i}\right)\left(q_{J_{i}}^{i}\right)=\left.\eta_{i, s}\right|_{I_{J_{i}}^{i}}
$$


for the boundary nodes; $i=1 \rightarrow N$. Here, as usual, $D_{s}: \underline{S}^{h} \rightarrow \underline{S}^{h}$ is defined component-wise. We then define $\vec{\theta}^{h}(t), \vec{\omega}_{d}^{h}(t) \in \underline{S}^{h}$ to be

$$
\vec{\theta}^{h}(t)=D_{s} \vec{X}^{h}(t) \quad \text { and } \quad \vec{\omega}_{d}^{h}(t)=\widehat{D}_{s} \vec{X}^{h}(t)=\frac{\vec{\theta}^{h}(t)}{\left|\vec{\theta}^{h}(t)\right|},
$$

which, on recalling assumption $\left(\mathcal{C}^{h}\right)$, are well-defined. We note for future reference that

$$
\left\langle\vec{X}_{s}^{h}, \vec{\xi}_{\Gamma^{h}}^{h}=\left\langle\vec{\theta}^{h}, \vec{\xi}\right\rangle_{\Gamma^{h}}^{h} \quad \forall \vec{\xi} \in \underline{S}^{h}\right.
$$

and similarly,

$$
\left\langle\left|\vec{\theta}^{h}\right|^{-1} \vec{\eta}_{s}, \vec{\xi}\right\rangle_{\Gamma^{h}}^{h},=\left\langle\widehat{D}_{s} \vec{\eta}, \vec{\xi}\right\rangle_{\Gamma^{h}}^{h} \quad \forall \vec{\eta}, \vec{\xi} \in \underline{S}^{h} .
$$

Let $\vec{Q}^{h}$ be defined by $\vec{Q}^{h}=\left(\vec{Q}_{1}^{h}, \ldots, \vec{Q}_{N}^{h}\right)$, where

$$
\vec{Q}_{i}^{h}(\rho, t)=\overrightarrow{I d}-\left[\vec{\omega}_{d, i}^{h}(\rho, t)\right] \otimes\left[\vec{\omega}_{d, i}^{h}(\rho, t)\right], \quad \rho \in[0,1], t \in[0, T], i=1 \rightarrow N .
$$

For future reference, we note also that for $\vec{\eta} \in \underline{S}^{h}$ it holds that

$$
\left[\frac{\delta}{\delta \vec{X}^{h}} \vec{\omega}_{d}^{h}\right](\vec{\eta})=\pi^{h}\left[\vec{Q}^{h}\left(\widehat{D}_{s} \vec{\eta}\right)\right] \quad \text { and hence } \quad\left[\vec{\omega}_{d}^{h}\right]_{t}=\pi^{h}\left[\vec{Q}^{h}\left(\widehat{D}_{s} \vec{X}_{t}^{h}\right)\right] .
$$

With these definitions it is easy to see that for any $\vec{\eta} \in \underline{S}^{h}$

$$
\begin{aligned}
\left\langle\left(\vec{Q}^{h} \vec{\eta}\right)_{t},\left(\overrightarrow{I d}-\vec{Q}^{h}\right) \vec{\eta}\right\rangle_{\Gamma^{h}}^{h} & =\left\langle\left(\vec{Q}^{h} \vec{\eta}\right)_{t},\left(\vec{\eta} \cdot \vec{\omega}_{d}^{h}\right) \vec{\omega}_{d}^{h}\right\rangle_{\Gamma^{h}}^{h}=-\left\langle\vec{Q}^{h} \vec{\eta},\left(\vec{\eta} \cdot \vec{\omega}_{d}^{h}\right)\left(\vec{\omega}_{d}^{h}\right)_{t}\right\rangle_{\Gamma^{h}}^{h} \\
& =-\left\langle\left|\vec{\theta}^{h}\right|^{-1}\left(\vec{\eta} \cdot \vec{\omega}_{d}^{h}\right) \vec{Q}^{h} \vec{\eta}, \vec{X}_{t, s}^{h}\right\rangle_{\Gamma^{h}}^{h} .
\end{aligned}
$$

In the planar case, $d=2$, we define $\vec{\nu}^{h}:=-\left(\vec{X}_{s}^{h}\right)^{\perp}$ and $\vec{\omega}^{h}:=-\left(\vec{\theta}^{h}\right)^{\perp} \in \underline{S}^{h}$, and set

$$
\vec{Q}_{\omega}^{h}(\rho, t):=\left[\vec{\omega}^{h}(\rho, t)\right] \otimes\left[\vec{\omega}^{h}(\rho, t)\right]=\left|\vec{\omega}^{h}(\rho, t)\right|^{2} \vec{Q}^{h}(\rho, t), \quad \rho \in[0,1], t \in[0, T] .
$$

Here we note that as in general $\left|\vec{\omega}_{i}^{h}\left(q_{j}^{i}, t\right)\right|<1$, the local operators $\vec{Q}_{\omega, i}^{h}$ in general are not projections. We note also for all $\vec{\chi}, \vec{\eta} \in \underline{S}^{h}$ that

$$
\left\langle\vec{\omega}^{h}, \vec{\chi}\right\rangle_{\Gamma^{h}}^{h}=\left\langle\vec{\nu}^{h}, \vec{\chi}\right\rangle_{\Gamma^{h}}^{h} \quad \text { and }\left\langle\vec{Q}_{\omega}^{h} \vec{\eta}, \vec{\chi}\right\rangle_{\Gamma^{h}}^{h}=\left\langle\vec{\eta} \cdot \omega^{h}, \vec{\chi} \cdot \vec{\nu}^{h}\right\rangle_{\Gamma^{h}}^{h} .
$$

It follows for all $\vec{\chi}, \vec{\eta} \in \underline{S}^{h}$ that

$$
\left\langle\left[\frac{\delta}{\delta \vec{X}^{h}} \vec{\omega}^{h}\right](\vec{\eta}), \vec{\chi}\right\rangle_{\Gamma^{h}}^{h}=-\left\langle\vec{\eta}_{s}^{\perp}+\left(\vec{\eta}_{s} \cdot \vec{X}_{s}^{h}\right) \vec{\omega}^{h}, \vec{\chi}\right\rangle_{\Gamma^{h}}^{h},
$$

and hence

$$
\left\langle\vec{\omega}_{t}^{h}, \vec{\chi}\right\rangle_{\Gamma^{h}}^{h}=-\left\langle\left(\vec{X}_{t, s}^{h}\right)^{\perp}+\left(\vec{X}_{t, s}^{h} \cdot \vec{X}_{s}^{h}\right) \vec{\omega}^{h}, \vec{\chi}\right\rangle_{\Gamma^{h}}^{h} .
$$


For later use we define

$$
\begin{array}{r}
\vec{Q}_{\star, i}^{h}(\rho, t):= \begin{cases}\vec{Q}_{i}^{h}(\rho, t) & \rho \in(0,1), \\
\overrightarrow{I d} & \rho \in\{0,1\},\end{cases} \\
\text { and, if } d=2, \quad \vec{Q}_{\omega, \star, i}^{h}(\rho, t):= \begin{cases}\vec{Q}_{\omega, i}^{h}(\rho, t) & \rho \in(0,1), \\
\overrightarrow{I d} & \rho \in\{0,1\},\end{cases}
\end{array}
$$

for $i=1 \rightarrow N$, which gives rise to the curve network versions $\vec{Q}_{\star}^{h}:=\left(\vec{Q}_{\star, 1}^{h}, \ldots, \vec{Q}_{\star, N}^{h}\right)$ and similarly $\vec{Q}_{\omega, \star}^{h}$. Fully discrete versions of these variants of the vertex based projections $\vec{Q}^{h}$ and $\vec{Q}_{\omega}^{h}$ will be instrumental in obtaining existence and uniqueness of the discrete solutions in some of our corresponding fully discrete schemes. Specifically for all those schemes, in which the trial spaces for $\vec{X}_{t}^{h}$ and $\vec{Y}^{h}$ differ. Moreover, in the case of two curves meeting at two $C^{1}$ junctions, the projections $\vec{Q}_{\star}^{h}$ and $\vec{Q}_{\omega, \star}^{h}$ will be crucial in obtaining semidiscrete finite element approximations with the desired equidistribution properties. In particular, these variants will prevent equidistribution being enforced across the two junction points, something that would often be in conflict with the flow to be approximated.

Let

$$
E_{\lambda}^{h}\left(\Gamma^{h}, \vec{\kappa}^{h}\right)=\frac{1}{2}\left\langle\varsigma \vec{\kappa}^{h}, \vec{\kappa}^{h}\right\rangle_{\Gamma^{h}}^{h}+\langle\varsigma, \lambda\rangle_{\Gamma^{h}}
$$

and

$$
E_{\lambda, \bar{\varkappa}}^{h}\left(\Gamma^{h}, \kappa^{h}\right)=\frac{1}{2}\left\langle\varsigma,\left(\kappa^{h}-\bar{\varkappa}\right)^{2}\right\rangle_{\Gamma^{h}}^{h}+\langle\varsigma, \lambda\rangle_{\Gamma^{h}}
$$

\subsection{The $C^{0}$ case - attachment only}

Similarly to the continuous setting in $\$ 2.1$, we consider the $L^{2}$-gradient flow of the discrete energy $E_{\lambda}^{h}\left(\Gamma^{h}, \vec{\kappa}^{h}\right)$ for $\vec{X}^{h} \in \underline{V}^{h}$ and $\vec{\kappa}^{h} \in \underline{W}^{h}$, subject to the side constraints

$$
\begin{array}{rlr}
\left\langle\vec{\kappa}^{h}, \vec{\eta}\right\rangle_{\Gamma^{h}}^{h}+\left\langle\vec{X}_{s}^{h}, \vec{\eta}_{s}\right\rangle_{\Gamma^{h}}=0 & \forall \vec{\eta} \in \underline{W^{h}} \\
\text { and } & \left.\forall \vec{\kappa}^{h} \cdot \vec{X}_{s}^{h}, \chi\right\rangle_{\Gamma^{h}}^{h}=0 & \forall \chi \in W^{h} .
\end{array}
$$

Hence $\vec{\kappa}^{h}$ is the natural discrete analogue of the curvature vector $\vec{\varkappa}$. Introducing the Lagrange multipliers $\vec{Y}^{h} \in \underline{W}^{h}$ and $Z^{h} \in W^{h}$ for (3.17a), (3.17b) we can define the discrete Lagrangian

$$
\begin{array}{r}
\mathcal{L}^{h}\left(\vec{X}^{h}, \vec{\kappa}^{h}, \vec{Y}^{h}, Z^{h}\right)=\frac{1}{2}\left\langle\varsigma \vec{\kappa}^{h}, \vec{\kappa}^{h}\right\rangle_{\Gamma^{h}}^{h}+\langle\varsigma, \lambda\rangle_{\Gamma^{h}}-\left\langle\vec{\kappa}^{h}, \vec{Y}^{h}\right\rangle_{\Gamma^{h}}^{h}-\left\langle\vec{X}_{s}^{h}, \vec{Y}_{s}^{h}\right\rangle_{\Gamma^{h}} \\
+\left\langle\vec{\kappa}^{h} \cdot \vec{X}_{s}^{h}, Z^{h}\right\rangle_{\Gamma^{h}}^{h}
\end{array}
$$

in analogue to $(2.29)$. Now taking variations $\left[\frac{\delta}{\delta \vec{X}^{h}} \mathcal{L}^{h}\right](\vec{\chi}),\left[\frac{\delta}{\delta \vec{\kappa}^{h}} \mathcal{L}^{h}\right](\vec{\xi}),\left[\frac{\delta}{\delta \vec{Y}^{h}} \mathcal{L}^{h}\right](\vec{\eta})$ and $\left[\frac{\delta}{\delta Z^{h}} \mathcal{L}^{h}\right](\chi)$ we obtain a discrete analogue of (2.30a)-(2.30d) for $\vec{\chi} \in \underline{V}^{h}, \vec{\xi} \in$ $\underline{W}^{h}, \vec{\eta} \in \underline{W}^{h}$ and $\chi \in W^{h}$. In particular, it follows that

$$
\pi^{h}\left[\vec{Q}_{i}^{h} \vec{Y}_{i}^{h}\right]=\varsigma_{i} \vec{\kappa}_{i}^{h} \quad \text { and } \quad Z_{i}^{h}=\pi^{h}\left[\vec{Y}_{i}^{h} \cdot \vec{X}_{i, s}^{h}\right], \quad i=1 \rightarrow N
$$


which means that $\vec{\kappa}^{h}$ and $Z^{h}$ can be eliminated from the derived variation $\left[\frac{\delta}{\delta \vec{X}^{h}} \mathcal{L}^{h}\right](\vec{\chi})$ in order to yield the desired weak formulation for the gradient flow of $E_{\lambda}^{h}\left(\Gamma^{h}, \vec{\kappa}^{h}\right)$.

In the case $d=2$, we consider the $L^{2}$-gradient flow of the discrete energy $E_{\lambda, \bar{\varkappa}}^{h}\left(\Gamma^{h}, \kappa^{h}\right)$ for $\vec{X}^{h} \in \underline{V}^{h}$ and $\left(\kappa^{h}-\bar{\varkappa}\right) \in W^{h}$, subject to the side constraint

$$
\left\langle\kappa^{h} \vec{\nu}^{h}, \vec{\eta}_{\Gamma^{h}}^{h}+\left\langle\vec{X}_{s}^{h}, \vec{\eta}_{s}\right\rangle_{\Gamma^{h}}=0 \quad \forall \vec{\eta} \in \underline{W}^{h} .\right.
$$

As before we can define the corresponding discrete Lagrangian, take variations and then eliminate $\kappa^{h}$ in terms of the Lagrange multiplier $\vec{Y}^{h} \in \underline{W}^{h}$ for (3.20).

Overall, the spatially discrete variants of (2.32a), (2.32b) and (2.37a), (2.37b) are given by the following two approximations. Given $\Gamma^{h}(0)=\vec{X}^{h}([0,1], 0)$, with $\vec{X}^{h}(0) \in \underline{V}^{h}$, for all $t \in(0, T]$ find $\Gamma^{h}(t)=\vec{X}^{h}([0,1], t)$ with $\vec{X}^{h}(t) \in \underline{V}^{h}$, and $\vec{Y}^{h}(t) \in \underline{W^{h}}$ such that either

$$
\begin{gathered}
\left\langle\vec{Q}_{\star}^{h} \vec{X}_{t}^{h}, \vec{\chi}\right\rangle_{\Gamma^{h}}^{h}-\left\langle\vec{\nabla}_{s} \vec{Y}^{h}, \vec{\nabla}_{s} \vec{\chi}\right\rangle_{\Gamma^{h}}-\frac{1}{2}\left\langle\left(\varsigma^{-1}\left|\vec{Q}^{h} \vec{Y}^{h}\right|^{2}-2 \varsigma \lambda\right) \vec{X}_{s}^{h}, \vec{\chi}_{s}\right\rangle_{\Gamma^{h}}^{h} \\
+\left\langle\varsigma^{-1}\left|\vec{\theta}^{h}\right|^{-1}\left(\vec{Y}^{h} \cdot \vec{\omega}_{d}^{h}\right) \vec{Q}^{h} \vec{Y}^{h}, \vec{\chi}_{s}\right\rangle_{\Gamma^{h}}^{h}=0 \quad \forall \vec{\chi} \in \underline{V}^{h} \\
\left\langle\varsigma^{-1} \vec{Q}^{h} \vec{Y}^{h}, \vec{\eta}\right\rangle_{\Gamma^{h}}^{h}+\left\langle\vec{X}_{s}^{h}, \vec{\eta}_{s}\right\rangle_{\Gamma^{h}}=0 \quad \forall \vec{\eta} \in \underline{W}^{h},
\end{gathered}
$$

or

$$
\begin{gathered}
\left\langle\vec{Q}_{\omega, \star}^{h} \vec{X}_{t}^{h}, \vec{\chi}\right\rangle_{\Gamma^{h}}^{h}-\left\langle\vec{\nabla}_{s} \vec{Y}^{h}, \vec{\nabla}_{s} \vec{\chi}\right\rangle_{\Gamma^{h}}+\frac{1}{2}\left\langle\left(\varsigma^{-1}\left(\vec{Y}^{h} \cdot \vec{\omega}^{h}\right)^{2}+2 \varsigma \lambda\right) \vec{X}_{s}^{h}, \vec{\chi}_{s}\right\rangle_{\Gamma^{h}}^{h} \\
-\left\langle\left(\varsigma^{-1} \vec{Y}^{h} \cdot \vec{\omega}^{h}+\bar{\varkappa}\right)\left(\vec{Y}^{h}\right)^{\perp}, \vec{\chi}_{s}\right\rangle_{\Gamma^{h}}^{h}=0 \quad \forall \vec{\chi} \in \underline{V}^{h}, \\
\left\langle\varsigma^{-1} \vec{Q}_{\omega}^{h} \vec{Y}^{h}, \vec{\eta}\right\rangle_{\Gamma^{h}}^{h}+\left\langle\vec{X}_{s}^{h}, \vec{\eta}_{s}\right\rangle_{\Gamma^{h}}=-\left\langle\bar{\varkappa} \vec{\omega}^{h}, \vec{\eta}\right\rangle_{\Gamma^{h}}^{h} \quad \forall \vec{\eta} \in \underline{W}^{h} .
\end{gathered}
$$

In the former case we have that $\vec{\kappa}_{i}^{h}:=\varsigma_{i}^{-1} \pi^{h}\left[\vec{Q}_{i}^{h} \vec{Y}_{i}^{h}\right]$ is the natural discrete approximation to $\vec{\varkappa}_{i}, i=1 \rightarrow N$, while in the latter case $\kappa_{i}^{h}:=\varsigma_{i}^{-1} \pi^{h}\left[\vec{Y}_{i}^{h} \cdot \vec{\omega}_{i}^{h}\right]+\bar{\varkappa}_{i}$ approximates $\varkappa_{i}, i=1 \rightarrow N$.

Remark 3.1. Replacing $\vec{Q}^{h} \vec{X}_{t}$ and $\vec{Q}_{\omega}^{h} \vec{X}_{t}$ in (3.21a) and (3.22a) with $\vec{Q}_{\star}^{h} \vec{X}_{t}$ and $\vec{Q}_{\omega, \star}^{h} \vec{X}_{t}$, respectively, is necessary in order to guarantee existence and uniqueness for the discrete solutions of the corresponding fully discrete schemes; see the proof of Theorem 4.1, below. Moreover, this replacement only means that rather than prescribing e.g. $\left(\vec{Q}^{h} \vec{X}_{t}\right)(0, t)$ at the junction, the full velocity vector $\vec{X}_{t}(0, t)$ is defined in (3.21a). This means that locally at that point, the approximation is similar in concept to the scheme in Ref. 10.

Theorem 3.1. Let $\left(\mathcal{C}^{h}\right)$ hold and let $\left(\vec{X}^{h}(t), \vec{Y}^{h}(t)\right)_{t \in(0, T]}$ be a solution to (3.21a), (3.21b). Then we have that

$$
\frac{\mathrm{d}}{\mathrm{d} t} E_{\lambda}^{h}\left(\Gamma^{h}, \varsigma^{-1} \vec{Q}^{h} \vec{Y}^{h}\right)=-\left\langle\vec{Q}_{\star}^{h} \vec{X}_{t}^{h}, \vec{Q}_{\star}^{h} \vec{X}_{t}^{h}\right\rangle_{\Gamma^{h}}^{h} \leq 0 .
$$

Moreover, if $d=2$ and $\left(\vec{X}^{h}(t), \vec{Y}^{h}(t)\right)_{t \in(0, T]}$ is a solution to (3.22a), (3.22b), then it holds that

$$
\frac{\mathrm{d}}{\mathrm{d} t} E_{\lambda, \bar{\varkappa}}^{h}\left(\Gamma^{h}, \varsigma^{-1} \vec{Y}^{h} \cdot \vec{\omega}^{h}+\bar{\varkappa}\right)=-\left\langle\vec{Q}_{\omega, \star}^{h} \vec{X}_{t}^{h}, \vec{X}_{t}^{h}\right\rangle_{\Gamma^{h}}^{h} \leq 0 .
$$


Proof. Differentiating (3.21b) with respect to $t$ and then choosing $\vec{\eta}=\vec{Y}^{h} \in \underline{W}^{h}$ yields that

$$
\left\langle\varsigma^{-1}\left(\vec{Q}^{h} \vec{Y}^{h}\right)_{t}, \vec{Y}^{h}\right\rangle_{\Gamma^{h}}^{h}+\left\langle\varsigma^{-1}\left|\vec{Q}^{h} \vec{Y}^{h}\right|^{2}, \vec{X}_{s}^{h} \cdot \vec{X}_{t, s}^{h}\right\rangle_{\Gamma^{h}}^{h}+\left\langle\vec{\nabla}_{s} \vec{X}_{t}^{h}, \vec{\nabla}_{s} \vec{Y}^{h}\right\rangle_{\Gamma^{h}}=0 .
$$

Combining (3.24) and (3.21a) with $\vec{\chi}=\vec{X}_{t}^{h} \in \underline{V}^{h}$ yields that

$$
\begin{aligned}
\left\langle\varsigma^{-1}\left(\vec{Q}^{h} \vec{Y}^{h}\right)_{t}, \vec{Y}^{h}\right\rangle_{\Gamma^{h}}^{h}+\frac{1}{2}\left\langle\varsigma^{-1}\left|\vec{Q}^{h} \vec{Y}^{h}\right|^{2}+2 \varsigma \lambda, \vec{X}_{s}^{h} \cdot \vec{X}_{t, s}^{h}\right\rangle_{\Gamma^{h}}^{h} \\
+\left\langle\varsigma^{-1}\left|\vec{\theta}^{h}\right|^{-1}\left(\vec{Y}^{h} \cdot \vec{\omega}_{d}^{h}\right) \vec{Q}^{h} \vec{Y}^{h}, \vec{X}_{t, s}^{h}\right\rangle_{\Gamma^{h}}^{h}=-\left\langle\vec{Q}_{\star}^{h} \vec{X}_{t}^{h}, \vec{Q}_{\star}^{h} \vec{X}_{t}^{h}\right\rangle_{\Gamma^{h}}^{h} .
\end{aligned}
$$

The desired result (3.23a) then follows from (3.25) on recalling (3.9). Moreover, differentiating (3.22b) with respect to $t$ and then choosing $\vec{\eta}=\vec{Y}^{h} \in \underline{W}^{h}$ yields that

$$
\begin{gathered}
\left\langle\varsigma^{-1}\left(\vec{Y}^{h} \cdot \vec{\omega}^{h}\right)_{t}, \vec{Y}^{h} \cdot \vec{\omega}^{h}\right\rangle_{\Gamma^{h}}^{h}+\left\langle\varsigma^{-1} \vec{Y}^{h} \cdot \vec{\omega}^{h}, \vec{Y}^{h} \cdot \vec{\nu}_{t}^{h}\right\rangle_{\Gamma^{h}}^{h} \\
\quad+\left\langle\varsigma^{-1}\left(\vec{Y}^{h} \cdot \vec{\omega}^{h}\right)\left(\vec{Y}^{h} \cdot \vec{\nu}^{h}\right), \vec{X}_{s}^{h} \cdot \vec{X}_{t, s}^{h}\right\rangle_{\Gamma^{h}}^{h}+\left\langle\vec{\nabla}_{s} \vec{X}_{t}^{h}, \vec{\nabla}_{s} \vec{Y}^{h}\right\rangle_{\Gamma^{h}} \\
=-\left\langle\vec{\nu}_{t}^{h}+\left(\vec{X}_{s}^{h} \cdot \vec{X}_{t, s}^{h}\right) \vec{\nu}^{h}, \vec{\varkappa} \vec{Y}^{h}\right\rangle_{\Gamma^{h}}^{h},
\end{gathered}
$$

where we have recalled (3.11). Combining (3.26) and (3.22a) with $\vec{\chi}=\vec{X}_{t}^{h} \in \underline{V}$ yields the desired result $(3.23 \mathrm{~b})$, on recalling that $\vec{\nu}_{i, t}^{h}=-\left(\vec{\nabla}_{s} \vec{X}_{i, t}^{h}\right)^{\perp}, i=1 \rightarrow N$, and that the natural discrete analogue of (2.44) holds.

Next we show an equidistribution property for the above schemes.

Theorem 3.2. Let $\left(\mathcal{C}^{h}\right)$ hold and let $\left(\vec{X}^{h}(t), \vec{Y}^{h}(t)\right)_{t \in(0, T]}$ denote a solution to (3.21a), (3.21b) or to (3.22a), (3.22b). For a fixed $i \in\{1, \ldots, N\}$ and a fixed time $t \in(0, T]$ let $\vec{a}_{j-\frac{1}{2}}^{h}:=\vec{X}_{i}^{h}\left(q_{j}^{i}, t\right)-\vec{X}_{i}^{h}\left(q_{j-1}^{i}, t\right), j=1 \rightarrow J_{i}$. Then it holds for $j=1 \rightarrow J_{i}-1$ that

$$
\left|\vec{a}_{j+\frac{1}{2}}^{h}\right|=\left|\vec{a}_{j-\frac{1}{2}}^{h}\right| \quad \text { if } \quad \vec{a}_{j+\frac{1}{2}}^{h} \nVdash \vec{a}_{j-\frac{1}{2}}^{h} .
$$

Proof. On choosing $\vec{\eta}=\vec{\omega}_{d, i}^{h}\left(q_{j}^{i}, t\right) \chi_{j}^{i} \in \underline{W}^{h}$, for $j=1 \rightarrow J_{i}-1$, in (3.21b), or in (3.22b), with $\chi_{j}^{i} \in W^{h}$ being the standard basis function associated with $q_{j}^{i}$, and on noting that $\vec{\omega}_{d, i}^{h}\left(q_{j}^{i}, t\right)=\frac{\vec{a}_{j+\frac{1}{2}}^{h}+\vec{a}_{j-\frac{1}{2}}^{h}}{\left|\vec{a}_{j+\frac{1}{2}}^{h}+\vec{a}_{j-\frac{1}{2}}^{h}\right|}$, it follows that

$$
\left(\frac{\vec{a}_{j+\frac{1}{2}}^{h}}{\left|\vec{a}_{j+\frac{1}{2}}^{h}\right|}-\frac{\vec{a}_{j-\frac{1}{2}}^{h}}{\left|\vec{a}_{j-\frac{1}{2}}^{h}\right|}\right) \cdot\left(\vec{a}_{j+\frac{1}{2}}^{h}+\vec{a}_{j-\frac{1}{2}}^{h}\right)=0, \quad j=1 \rightarrow J_{i}-1 .
$$

Clearly, (3.28) and the Cauchy-Schwarz inequality imply that $\left|\vec{a}_{j+\frac{1}{2}}^{h}\right|=\left|\vec{a}_{j-\frac{1}{2}}^{h}\right|$ if $\vec{a}_{j+\frac{1}{2}}^{h}$ is not parallel to $\vec{a}_{j-\frac{1}{2}}^{h}$. 


\subsection{The $C^{1}$ case - enforcing Young's law}

In contrast to $\S 3.1$, here we need to distinguish two cases. We will present two approximations for the case $N \geq 3$, and two approximations for the case $N=2$. In each case one approximation will be for planar flows in the presence of spontaneous curvatures, and one for higher codimension flows in $\mathbb{R}^{d}, d \geq 2$. In terms of presenting our results it would be very desirable to cut down on the number of schemes to be investigated. However, each individual case has features which make it impossible to unify the presentation further.

We begin with the case of $N \geq 3$ curves meeting at two junctions. Then it is straightforward to mimic the procedure in $\S 2.2$ on the discrete level, similarly to §3.1. In particular, as an auxiliary variable we introduce $\vec{U}^{h} \in \underline{V}^{h}$ such that

$$
\pi^{h}\left[\vec{Q}_{i}^{h} \vec{U}_{i}^{h}\right]=\varsigma_{i} \vec{\kappa}_{i}^{h}, \quad i=1 \rightarrow N .
$$

Then we formally consider the $L^{2}$-gradient flow of the discrete energy $E_{\lambda}^{h}\left(\Gamma^{h}, \varsigma^{-1} \vec{Q}^{h} \vec{U}^{h}\right)$ for $\vec{X}^{h} \in \underline{V}^{h}$ and $\vec{U}^{h} \in \underline{V}^{h}$ under the side constraint

$$
\left\langle\varsigma^{-1} \vec{Q}^{h} \vec{U}^{h}, \vec{\eta}\right\rangle_{\Gamma^{h}}^{h}+\left\langle\vec{X}_{s}^{h}, \vec{\eta}_{s}\right\rangle_{\Gamma^{h}}=0 \quad \forall \vec{\eta} \in \underline{V}^{h} .
$$

As in the continuous case, we can now define the corresponding discrete Lagrangian $\mathcal{L}^{h}\left(\vec{X}^{h}, \vec{U}^{h}, \vec{Y}^{h}\right):=\frac{1}{2}\left\langle\varsigma^{-1},\left|\vec{Q}^{h} \vec{U}^{h}\right|^{2}\right\rangle_{\Gamma^{h}}^{h}+\langle\varsigma, \lambda\rangle_{\Gamma^{h}}-\left\langle\varsigma^{-1} \vec{Q}^{h} \vec{U}^{h}, \vec{Y}^{h}\right\rangle_{\Gamma^{h}}^{h}-\left\langle\vec{X}_{s}^{h}, \vec{Y}_{s}^{h}\right\rangle_{\Gamma^{h}}$,

where $\vec{Y}^{h} \in \underline{V}^{h}$ is a Lagrange multiplier for (3.30). On taking the appropriate variations, and mimicking the procedure on the continuous level, we obtain that

$$
\pi^{h}\left[\vec{Q}_{i}^{h} \vec{Y}_{i}^{h}\right]=\pi^{h}\left[\vec{Q}_{i}^{h} \vec{U}_{i}^{h}\right]=\varsigma_{i} \vec{\kappa}_{i}^{h}, \quad i=1 \rightarrow N .
$$

Hence we can eliminate $\vec{U}^{h}$ from the variation $\left[\frac{\delta}{\delta \vec{X}^{h}} \mathcal{L}^{h}\right](\vec{\chi})$ in order to yield the desired weak formulation for the gradient flow of $E_{\lambda}^{h}\left(\Gamma^{h}, \varsigma^{-1} \vec{Q}^{h} \vec{U}^{h}\right)$.

Similarly, in the case $d=2$ for $N \geq 3$, we introduce $\vec{U}^{h} \in \underline{V}^{h}$ such that

$$
\pi^{h}\left[\vec{Q}_{\omega, i}^{h} \vec{U}_{i}^{h}\right]=\varsigma_{i} \pi^{h}\left[\left(\kappa_{i}^{h}-\bar{\varkappa}_{i}\right) \vec{\omega}_{i}^{h}\right], \quad i=1 \rightarrow N,
$$

and then consider the $L^{2}$-gradient flow of the discrete energy $E_{\lambda, \bar{\varkappa}}^{h}\left(\Gamma^{h}, \varsigma^{-1} \vec{U}^{h} \cdot \vec{\omega}^{h}+\right.$ $\bar{\varkappa})$ under the side constraint

$$
\left\langle\varsigma^{-1} \vec{Q}_{\omega}^{h} \vec{U}^{h}, \vec{\eta}\right\rangle_{\Gamma^{h}}^{h}+\left\langle\vec{X}_{s}^{h}, \vec{\eta}_{s}\right\rangle_{\Gamma^{h}}=-\left\langle\bar{\varkappa} \vec{\omega}^{h}, \vec{\eta}\right\rangle_{\Gamma^{h}}^{h} \quad \forall \vec{\eta} \in \underline{V}^{h} .
$$

Introducing the Lagrange multiplier $\vec{Y}^{h} \in \underline{V}^{h}$ for (3.34), we define the discrete Lagrangian

$$
\begin{array}{r}
\mathcal{L}^{h}\left(\vec{X}^{h}, \vec{U}^{h}, \vec{Y}^{h}\right):=\frac{1}{2}\left\langle\varsigma^{-1},\left(\vec{U}^{h} \cdot \vec{\omega}^{h}\right)^{2}\right\rangle_{\Gamma^{h}}^{h}+ \\
-\langle\varsigma, \lambda\rangle_{\Gamma^{h}}-\left\langle\varsigma^{-1} \vec{Q}_{\omega}^{h} \vec{U}^{h}, \vec{Y}^{h}\right\rangle_{\Gamma^{h}}^{h} \\
-\left\langle\vec{X}_{s}^{h}, \vec{Y}_{s}^{h}\right\rangle_{\Gamma^{h}}-\left\langle\bar{\varkappa} \vec{\omega}^{h}, \vec{Y}^{h}\right\rangle_{\Gamma^{h}}^{h} .
\end{array}
$$

On taking the appropriate variations, and mimicking the procedure on the continuous level and noting (3.12), we obtain that $\pi^{h}\left[\vec{Q}_{\omega}^{h} \vec{Y}^{h}\right]=\pi^{h}\left[\vec{Q}_{\omega}^{h} \vec{U}^{h}\right]$, which allows us to eliminate $\vec{U}^{h}$ from the variation $\left[\frac{\delta}{\delta \vec{X}^{h}} \mathcal{L}^{h}\right](\vec{\chi})$ in order to yield the desired weak formulation for the considered gradient flow. 
Overall, the spatially discrete variants of (2.53a), (2.53b) and (2.58a), (2.58b) for $N \geq 3$ are then given by the following two approximations. Given $\Gamma^{h}(0)=$ $\vec{X}^{h}([0,1], 0)$, with $\vec{X}^{h}(0) \in \underline{V}^{h}$, for all $t \in(0, T]$ find $\Gamma^{h}(t)=\vec{X}^{h}([0,1], t)$ with $\vec{X}^{h}(t) \in \underline{V}^{h}$, and $\vec{Y}^{h}(t) \in \underline{V}^{h}$ such that either

$$
\begin{gathered}
\left\langle\vec{Q}^{h} \vec{X}_{t}^{h}, \vec{\chi}\right\rangle_{\Gamma^{h}}^{h}-\left\langle\vec{\nabla}_{s} \vec{Y}^{h}, \vec{\nabla}_{s} \vec{\chi}\right\rangle_{\Gamma^{h}}-\frac{1}{2}\left\langle\left(\varsigma^{-1}\left|\vec{Q}^{h} \vec{Y}^{h}\right|^{2}-2 \varsigma \lambda\right) \vec{X}_{s}^{h}, \vec{\chi}_{s}\right\rangle_{\Gamma^{h}}^{h} \\
+\left\langle\varsigma^{-1}\left|\vec{\theta}^{h}\right|^{-1}\left(\vec{Y}^{h} \cdot \vec{\omega}_{d}^{h}\right) \vec{Q}^{h} \vec{Y}^{h}, \vec{\chi}_{s}\right\rangle_{\Gamma^{h}}^{h}=0 \quad \forall \vec{\chi} \in \underline{V}^{h}
\end{gathered}
$$

or

$$
\begin{aligned}
\left\langle\vec{Q}_{\omega}^{h} \vec{X}_{t}^{h}, \vec{\chi}\right\rangle_{\Gamma^{h}}^{h}-\left\langle\vec{\nabla}_{s} \vec{Y}^{h}, \vec{\nabla}_{s} \vec{\chi}\right\rangle_{\Gamma^{h}}+ & \frac{1}{2}\left\langle\left(\varsigma^{-1}\left(\vec{Y}^{h} \cdot \vec{\omega}^{h}\right)^{2}+2 \varsigma \lambda\right) \vec{X}_{s}^{h}, \vec{\chi}_{s}\right\rangle_{\Gamma^{h}}^{h} \\
& -\left\langle\left(\varsigma^{-1} \vec{Y}^{h} \cdot \vec{\omega}^{h}+\bar{\varkappa}\right)\left(\vec{Y}^{h}\right)^{\perp}, \vec{\chi}_{s}\right\rangle_{\Gamma^{h}}^{h}=0 \quad \forall \\
\left\langle\varsigma^{-1} \vec{Q}_{\omega}^{h} \vec{Y}^{h}, \vec{\eta}\right\rangle_{\Gamma^{h}}^{h}+\left\langle\vec{X}_{s}^{h}, \vec{\eta}_{s}\right\rangle_{\Gamma^{h}}=-\left\langle\vec{\varkappa} \vec{\omega}^{h}, \vec{\eta}\right\rangle_{\Gamma^{h}}^{h} \quad \forall \vec{\eta} \in \underline{V}^{h} . & \text { (3.37a) }
\end{aligned},
$$

In the former case we have that $\vec{\kappa}_{i}^{h}:=\varsigma_{i}^{-1} \pi^{h}\left[\vec{Q}_{i}^{h} \vec{Y}_{i}^{h}\right]$ is the natural discrete approximation to $\vec{\varkappa}_{i}, i=1 \rightarrow N$, while in the latter case $\kappa_{i}^{h}:=\varsigma_{i}^{-1} \pi^{h}\left[\vec{Y}_{i}^{h} \cdot \vec{\omega}_{i}^{h}\right]+\bar{\varkappa}_{i}$ approximates $\varkappa_{i}, i=1 \rightarrow N$.

Theorem 3.3. Let $N \geq 3$. Let $\left(\mathcal{C}^{h}\right)$ hold and let $\left(\vec{X}^{h}(t), \vec{Y}^{h}(t)\right)_{t \in(0, T]}$ be a solution to (3.36a), (3.36b). Then we have that

$$
\frac{\mathrm{d}}{\mathrm{d} t} E_{\lambda}^{h}\left(\Gamma^{h}, \varsigma^{-1} \vec{Q}^{h} \vec{Y}^{h}\right)=-\left\langle\vec{Q}^{h} \vec{X}_{t}^{h}, \vec{Q}^{h} \vec{X}_{t}^{h}\right\rangle_{\Gamma^{h}}^{h} \leq 0 .
$$

Moreover, if $d=2$ and $\left(\vec{X}^{h}(t), \vec{Y}^{h}(t)\right)_{t \in(0, T]}$ is a solution to (3.37a), (3.37b), then it holds that

$$
\frac{\mathrm{d}}{\mathrm{d} t} E_{\lambda, \bar{\varkappa}}^{h}\left(\Gamma^{h}, \varsigma^{-1} \vec{Y}^{h} \cdot \vec{\omega}^{h}+\bar{\varkappa}\right)=-\left\langle\vec{Q}_{\omega}^{h} \vec{X}_{t}^{h}, \vec{X}_{t}^{h}\right\rangle_{\Gamma^{h}}^{h} \leq 0
$$

Proof. The proof is analogous to the proof of Theorem 3.1.

Theorem 3.4. Let $N \geq 3$. Let $\left(\mathcal{C}^{h}\right)$ hold and let $\left(\vec{X}^{h}(t), \vec{Y}^{h}(t)\right)_{t \in(0, T]}$ denote a solution to (3.36a), (3.36b) or to (3.37a), (3.37b). For a fixed $i \in\{1, \ldots, N\}$ and a fixed time $t \in(0, T]$ let $\vec{a}_{j-\frac{1}{2}}^{h}:=\vec{X}_{i}^{h}\left(q_{j}^{i}, t\right)-\vec{X}_{i}^{h}\left(q_{j-1}^{i}, t\right), j=1 \rightarrow J_{i}$. Then it holds for $j=1 \rightarrow J_{i}-1$ that

$$
\left|\vec{a}_{j+\frac{1}{2}}^{h}\right|=\left|\vec{a}_{j-\frac{1}{2}}^{h}\right| \quad \text { if } \quad \vec{a}_{j+\frac{1}{2}}^{h} \nVdash \vec{a}_{j-\frac{1}{2}}^{h} .
$$

Proof. Similarly to the proof of Theorem 3.2, choosing $\vec{\eta}=\vec{\omega}_{d, i}^{h}\left(q_{j}^{i}, t\right) \chi_{j}^{i} \in \underline{W}^{h} \subset$ $\underline{V}^{h}$, for $j=1 \rightarrow J_{i}-1$, in (3.36b), or in (3.37b), we obtain (3.28), and hence the desired result follows.

Remark 3.2. Looking carefully at the proof of Theorem 3.4, and noting that for $N \geq 3$ in general $\vec{\omega}_{d}^{h}(t) \notin \underline{V}^{h}$, we see, for example, that on choosing any $\vec{\eta} \in$ 
$\underline{V}^{h}$ with $\vec{\eta}_{1}=\vec{\omega}_{d, 1}^{h}(0, t) \chi_{0}^{1}$ in the proof will yield no information on the segment length $\left|\vec{X}_{1}^{h}\left(q_{1}^{1}, t\right)-\vec{X}_{1}^{h}\left(q_{0}^{1}, t\right)\right|$. However, in the special case $N=2$ the approximation (3.36a), (3.36b), say, will weakly enforce

$$
\vec{\omega}_{d, 1}^{h}(r, t)+\vec{\omega}_{d, 2}^{h}(r, t) \approx \overrightarrow{0}, \quad r \in\{0,1\} .
$$

Hence the function $\vec{\xi}:=\vec{\omega}_{d, 1}^{h}(0, t) \chi_{0}^{1}-\vec{\omega}_{d, 2}^{h}(0, t) \chi_{0}^{2}$ will almost be an element of $\underline{V}^{h}$. If $\vec{\xi} \in \underline{V}^{h}$, then we could choose $\vec{\eta}=\vec{\xi}$ in, say, (3.36b) in order to obtain that

$$
\left|\vec{X}_{1}^{h}\left(q_{1}^{1}, t\right)-\vec{X}_{1}^{h}\left(q_{0}^{1}, t\right)\right|=\left|\vec{X}_{2}^{h}\left(q_{1}^{2}, t\right)-\vec{X}_{2}^{h}\left(q_{0}^{2}, t\right)\right|,
$$

unless these two segments are parallel, and similarly at the other junction. Together with the results from Theorem 3.4, the condition (3.41) would imply a global equidistribution property across the two curves $\Gamma_{1}^{h}$ and $\Gamma_{2}^{h}$. Clearly, as the number of mesh points is fixed on each $\Gamma_{i}^{h}$, enforcing equidistribution across the two curves would fix the relative lengths of the curves to each other. This constraint would be so strong, that the scheme would not be able to approximate most flows; and we do observe this behaviour in practice for a fully discrete approximation that is based on (3.36a), (3.36b). See Figure 6 below for an example. It is for this reason that we have to replace $\vec{Q}^{h}$ and $\vec{Q}_{\omega}^{h}$ with $\vec{Q}_{\star}^{h}$ and $\vec{Q}_{\omega, \star}^{h}$ in (3.36b) and (3.37b), respectively; recall (3.14).

Of course, there is a contradiction in our derivation of (3.41). On the one hand, we can only derive (3.41) if $\vec{\xi} \in \underline{V}^{h}$; that is, (3.40) holds with equality. On the other hand, (3.41) is only guaranteed, if the two segments are not parallel, i.e. if (3.40) does not hold with equality. Clearly, this is a contradiction. Nevertheless, numerical evidence for the fully discrete variants of e.g. (3.36a), (3.36b) suggests that if we replace $\vec{\xi}$ in the above argument with $\vec{\omega}_{d, 1}^{h}(0, t) \chi_{0}^{1}-\vec{\omega}_{d, 1}^{h}(0, t) \chi_{0}^{2} \in \underline{V}^{h}$, then, even if (3.40) does not hold with equality, we obtain an algebraic condition very close to (3.41). This algebraic condition then appears to lead to something very close to equidistribution, as evidenced by our numerical experiments.

We now consider the case $N=2$. In view of Remark 3.2 we will replace $\vec{Q}^{h}$ with $\vec{Q}_{\star}^{h}$ in (3.29). This mimics on the discrete level what we discussed in (2.46), (2.47) on the continuous level.

We begin by introducing the auxiliary variable $\vec{U}^{h} \in \underline{V}^{h}$ such that

$$
\pi^{h}\left[\vec{Q}_{\star, i}^{h} \vec{U}_{i}^{h}\right]=\varsigma_{i} \vec{\kappa}_{i}^{h}, \quad i=1 \rightarrow 2 .
$$

Similarly to (2.49) we then introduce the Lagrangian

$$
\mathcal{L}^{h}\left(\vec{X}^{h}, \vec{U}^{h}, \vec{Y}^{h}\right):=\frac{1}{2}\left\langle\varsigma^{-1},\left|\vec{Q}_{\star}^{h} \vec{U}^{h}\right|^{2}\right\rangle_{\Gamma^{h}}^{h}+\langle\varsigma, \lambda\rangle_{\Gamma^{h}}-\left\langle\varsigma^{-1} \vec{Q}_{\star}^{h} \vec{U}^{h}, \vec{Y}^{h}\right\rangle_{\Gamma^{h}}^{h}-\left\langle\vec{X}_{s}^{h}, \vec{Y}_{s}^{h}\right\rangle_{\Gamma^{h}} .
$$


Taking variations, we obtain the gradient flow

$$
\begin{array}{r}
\left\langle\vec{Q}_{\star}^{h} \vec{X}_{t}^{h}, \vec{\chi}\right\rangle_{\Gamma^{h}}^{h} \\
=\left\langle\vec{\nabla}_{s} \vec{Y}^{h}, \vec{\nabla}_{s} \vec{\chi}\right\rangle_{\Gamma^{h}}-\frac{1}{2}\left\langle\left(\varsigma^{-1}\left|\vec{Q}_{\star}^{h} \vec{U}^{h}\right|^{2}-2 \varsigma^{-1} \vec{Q}_{\star}^{h} \vec{U}^{h} \cdot \vec{Y}^{h}+2 \varsigma \lambda\right) \vec{X}_{s}^{h}, \vec{\chi}_{s}\right\rangle_{\Gamma^{h}}^{h} \\
+\left\langle\varsigma^{-1}\left(\vec{Y}^{h}-\vec{Q}_{\star}^{h} \vec{U}^{h}\right), \pi_{\underline{W}}^{h}\left[\left[\frac{\delta}{\delta \vec{X}^{h}} \vec{Q}^{h} \vec{U}^{h}\right](\vec{\chi})\right]\right\rangle_{\Gamma^{h}}^{h} \forall \vec{\chi} \in \underline{V}^{h}, \quad \text { (3.44a) } \\
\left\langle\varsigma^{-1} \vec{Q}_{\star}^{h} \vec{U}^{h}, \vec{\xi}\right\rangle_{\Gamma^{h}}^{h}-\left\langle\varsigma^{-1} \vec{Y}^{h}, \vec{Q}_{\star}^{h} \vec{\xi}\right\rangle_{\Gamma^{h}}^{h}=0 \quad \forall \vec{\xi} \in \underline{V}^{h}, \\
\left\langle\varsigma^{-1} \vec{Q}_{\star}^{h} \vec{U}^{h}, \vec{\eta}\right\rangle_{\Gamma^{h}}^{h}+\left\langle\vec{X}_{s}^{h}, \vec{\eta}_{s}\right\rangle_{\Gamma^{h}}=0 \quad \forall \vec{\eta} \in \underline{V}^{h},
\end{array}
$$

where $\pi_{\underline{W}}^{h}:\left[C\left([0,1], \mathbb{R}^{d}\right)\right]^{N} \rightarrow \underline{W^{h}}$ is the standard Lagrange interpolation operator with zero Dirichlet boundary conditions. We observe that we could have chosen $\vec{Q}^{h} \vec{X}_{t}^{h}$ in place of $\vec{Q}_{\star}^{h} \vec{X}_{t}^{h}$ in (3.44a). However, similarly to Remark 3.1 this has little effect in practice, and we prefer (3.44a) and the subsequent weak formulation due to its symmetry. It follows from (3.44b) and (3.42) that

$$
\pi^{h}\left[\vec{Q}_{\star, i}^{h} \vec{Y}_{i}^{h}\right]=\pi^{h}\left[\vec{Q}_{\star, i}^{h} \vec{U}_{i}^{h}\right]=\varsigma_{i} \vec{\kappa}_{i}^{h}, \quad i=1 \rightarrow 2 .
$$

Similarly to $\S 2.2$, it is now possible to eliminate $\vec{U}^{h}$ from (3.44a), (3.44c) as follows. First, in a discrete analogue to (2.51), it follows from (3.8) that

$$
\left[\frac{\delta}{\delta \vec{X}^{h}} \vec{Q}^{h} \vec{U}^{h}\right](\vec{\chi})=-\left(\vec{U}^{h} \cdot \vec{\omega}_{d}^{h}\right) \pi^{h}\left[\vec{Q}^{h}\left(\widehat{D}_{s} \vec{\chi}\right)\right]-\vec{U}^{h} \cdot \pi^{h}\left[\vec{Q}^{h}\left(\widehat{D}_{s} \vec{\chi}\right)\right] \vec{\omega}_{d}^{h} .
$$

Therefore, on noting (3.45) and on recalling (3.6) we obtain for the last term in (3.44a) that

$$
\begin{aligned}
\left\langle\varsigma^{-1}\left(\vec{Y}^{h}-\vec{Q}_{\star}^{h} \vec{U}^{h}\right), \pi_{\underline{W}}^{h}\left[\left[\frac{\delta}{\delta \vec{X}^{h}} \vec{Q}^{h} \vec{U}^{h}\right](\vec{\chi})\right]\right\rangle_{\Gamma^{h}}^{h} \\
\quad=-\left\langle\varsigma^{-1} \pi_{W}^{h}\left[\left|\vec{\theta}^{h}\right|^{-1}\left(\vec{Y}^{h} \cdot \vec{\omega}_{d}^{h}\right)\right] \vec{Q}_{\star}^{h} \vec{Y}^{h}, \vec{\chi}_{s}\right\rangle_{\Gamma^{h}}^{h} .
\end{aligned}
$$

where $\pi_{W}^{h}:[C([0,1], \mathbb{R})]^{N} \rightarrow W^{h}$ is defined similarly to $\pi_{\underline{W}}^{h}$.

Similarly, in the case $d=2$ and $N=2$ for the discrete energy $E_{\lambda, \bar{\varkappa}}^{h}\left(\Gamma^{h}, \kappa^{h}\right)$, we introduce $\vec{U}^{h} \in \underline{V}^{h}$ such that

$$
\pi^{h}\left[\vec{Q}_{\omega, \star, i}^{h} \vec{U}_{i}^{h}\right] \text { replaces } \varsigma_{i} \pi^{h}\left[\left(\kappa_{i}^{h}-\bar{\varkappa}_{i}\right) \vec{\omega}_{i}^{h}\right], \quad i=1 \rightarrow 2,
$$

to obtain the corresponding Lagrangian

$$
\begin{array}{r}
\mathcal{L}^{h}\left(\vec{X}^{h}, \vec{U}^{h}, \vec{Y}^{h}\right):=\frac{1}{2}\left\langle\varsigma^{-1} \vec{Q}_{\omega, \star}^{h} \vec{U}^{h}, \vec{U}^{h}\right\rangle_{\Gamma^{h}}^{h}+\langle\varsigma, \lambda\rangle_{\Gamma^{h}}-\left\langle\varsigma^{-1} \vec{Q}_{\omega, \star}^{h} \vec{U}^{h}, \vec{Y}^{h}\right\rangle_{\Gamma^{h}}^{h} \\
-\left\langle\vec{X}_{s}^{h}, \vec{Y}_{s}^{h}\right\rangle_{\Gamma^{h}} .
\end{array}
$$

Once again on taking variations, we arrive at the analogue of $(3.45), \pi^{h}\left[\vec{Q}_{\omega, \star}^{h} \vec{U}^{h}\right]=$ $\pi^{h}\left[\vec{Q}_{\omega, \star}^{h} \vec{Y}^{h}\right]$, for the associated Lagrange multiplier $\vec{Y}^{h} \in \underline{V}^{h}$. We note that, in contrast to (3.42), we do not state equality in (3.46), as this would require that $\vec{\omega}_{1}^{h}$ and $\vec{\omega}_{2}^{h}$ are parallel at the junctions, which is not true in general. 
Overall our semidiscrete approximations in the case $N=2$ can then be formulated as follows. Given $\Gamma^{h}(0)=\vec{X}^{h}([0,1], 0)$, with $\vec{X}^{h}(0) \in \underline{V}^{h}$, for all $t \in(0, T]$ find $\Gamma^{h}(t)=\vec{X}^{h}([0,1], t)$ with $\vec{X}^{h}(t) \in \underline{V}^{h}$, and $\vec{Y}^{h}(t) \in \underline{V}^{h}$ such that either

$$
\begin{gathered}
\left\langle\vec{Q}_{\star}^{h} \vec{X}_{t}^{h}, \vec{\chi}\right\rangle_{\Gamma^{h}}^{h}-\left\langle\vec{\nabla}_{s} \vec{Y}^{h}, \vec{\nabla}_{s} \vec{\chi}\right\rangle_{\Gamma^{h}}-\frac{1}{2}\left\langle\left(\varsigma^{-1}\left|\vec{Q}_{\star}^{h} \vec{Y}^{h}\right|^{2}-2 \varsigma \lambda\right) \vec{X}_{s}^{h}, \vec{\chi}_{s}\right\rangle_{\Gamma^{h}}^{h} \\
+\left\langle\varsigma^{-1} \pi_{W}^{h}\left[\left|\vec{\theta}^{h}\right|^{-1}\left(\vec{Y}^{h} \cdot \vec{\omega}_{d}^{h}\right)\right] \vec{Q}_{\star}^{h} \vec{Y}^{h}, \vec{\chi}_{s}\right\rangle_{\Gamma^{h}}^{h}=0 \quad \forall \vec{\chi} \in \underline{V}^{h}, \\
\left\langle\varsigma^{-1} \vec{Q}_{\star}^{h} \vec{Y}^{h}, \vec{\eta}\right\rangle_{\Gamma^{h}}^{h}+\left\langle\vec{X}_{s}^{h}, \vec{\eta}_{s}\right\rangle_{\Gamma^{h}}=0 \quad \forall \vec{\eta} \in \underline{V}^{h},
\end{gathered}
$$

or

$$
\begin{aligned}
\left\langle\vec{Q}_{\omega, \star}^{h} \vec{X}_{t}^{h}, \vec{\chi}\right\rangle_{\Gamma^{h}}^{h} & -\left\langle\vec{\nabla}_{s} \vec{Y}^{h}, \vec{\nabla}_{s} \vec{\chi}\right\rangle_{\Gamma^{h}} \\
& +\frac{1}{2}\left\langle\left(2 \varsigma^{-1} \pi_{W}^{h}\left[\left(\vec{Y}^{h} \cdot \vec{\omega}^{h}\right)^{2}\right]-\varsigma^{-1}\left(\vec{Q}_{\omega, \star}^{h} \vec{Y}^{h}\right) \cdot \vec{Y}^{h}+2 \varsigma \lambda\right) \vec{X}_{s}^{h}, \vec{\chi}_{s}\right\rangle_{\Gamma^{h}}^{h} \\
& -\left\langle\left(\varsigma^{-1} \pi_{W}^{h}\left[\vec{Y}^{h} \cdot \vec{\omega}^{h}\right]+\bar{\varkappa}\right)\left(\vec{Y}^{h}\right)^{\perp}, \vec{\chi}_{s}\right\rangle_{\Gamma^{h}}^{h}=0 \quad \forall \vec{\chi} \in \underline{V}^{h},
\end{aligned}
$$

In the former case we have that $\vec{\kappa}_{i}^{h}:=\varsigma_{i}^{-1} \pi^{h}\left[\vec{Q}_{\star, i}^{h} \vec{Y}_{i}^{h}\right]$ is the natural discrete approximation to $\vec{\varkappa}_{i}, i=1 \rightarrow 2$, while in the latter case $\kappa_{i}^{h}:=\varsigma_{i}^{-1} \pi^{h}\left[\vec{Y}_{i}^{h} \cdot \vec{\omega}_{i}^{h}\right]+\bar{\varkappa}_{i}$ approximates $\varkappa_{i}, i=1 \rightarrow 2$.

Before we state the stability results for (3.48a), (3.48b) and (3.49a), (3.49b), we remark that due to the form of the Lagrangian (3.47), the correct energy to consider for the latter scheme is

$$
\begin{aligned}
\frac{1}{2}\left\langle\varsigma^{-1} \vec{Q}_{\omega, \star}^{h} \vec{Y}^{h}, \vec{Y}^{h}\right\rangle_{\Gamma^{h}}^{h}+\langle\varsigma, \lambda\rangle_{\Gamma^{h}}^{h} \\
\quad=E_{\lambda, \bar{\varkappa}}^{h}\left(\Gamma^{h}, \varsigma^{-1} \vec{Y}^{h} \cdot \vec{\omega}^{h}+\bar{\varkappa}\right)+\left\langle\varsigma^{-1},\left(I-\pi_{W}^{h}\right)\left(\vec{Y}^{h} \cdot\left[\vec{\omega}^{h}\right]^{\perp}\right)^{2}\right\rangle_{\Gamma^{h}}^{h} ;
\end{aligned}
$$

i.e. the energy in $(3.38 \mathrm{~b})$ is supplemented with a nonnegative contribution from the tangential components of $\vec{Y}^{h}$ at the two junctions. Of course, if equality did hold in (3.46) then these tangential components would vanish.

Theorem 3.5. Let $N=2$. Let $\left(\mathcal{C}^{h}\right)$ hold and let $\left(\vec{X}^{h}(t), \vec{Y}^{h}(t)\right)_{t \in(0, T]}$ be a solution to (3.48a), (3.48b). Then we have that

$$
\frac{\mathrm{d}}{\mathrm{d} t} E_{\lambda}^{h}\left(\Gamma^{h}, \varsigma^{-1} \vec{Q}_{\star}^{h} \vec{Y}^{h}\right)=-\left\langle\vec{Q}_{\star}^{h} \vec{X}_{t}^{h}, \vec{Q}_{\star}^{h} \vec{X}_{t}^{h}\right\rangle_{\Gamma^{h}}^{h} \leq 0 .
$$

Moreover, if $d=2$ and $\left(\vec{X}^{h}(t), \vec{Y}^{h}(t)\right)_{t \in(0, T]}$ is a solution to (3.49a), (3.49b), then it holds that

$$
\frac{\mathrm{d}}{\mathrm{d} t}\left[\frac{1}{2}\left\langle\varsigma^{-1} \vec{Q}_{\omega, \star}^{h} \vec{Y}^{h}, \vec{Y}^{h}\right\rangle_{\Gamma^{h}}^{h}+\langle\varsigma, \lambda\rangle_{\Gamma^{h}}^{h}\right]=-\left\langle\vec{Q}_{\omega, \star}^{h} \vec{X}_{t}^{h}, \vec{X}_{t}^{h}\right\rangle_{\Gamma^{h}}^{h} \leq 0 .
$$

Proof. Differentiating (3.48b) with respect to $t$ and then choosing $\vec{\eta}=\vec{Y}^{h} \in \underline{V}^{h}$, and combining this with (3.48a) with $\vec{\chi}=\vec{X}_{t}^{h} \in \underline{V}^{h}$ yields, similarly to (3.25), that

$$
\begin{aligned}
& \left\langle\varsigma^{-1}\left(\vec{Q}_{\star}^{h} \vec{Y}^{h}\right)_{t}, \vec{Y}^{h}\right\rangle_{\Gamma^{h}}^{h}+\frac{1}{2}\left\langle\varsigma^{-1}\left|\vec{Q}_{\star}^{h} \vec{Y}^{h}\right|^{2}+2 \varsigma \lambda, \vec{X}_{s}^{h} \cdot \vec{X}_{t, s}^{h}\right\rangle_{\Gamma^{h}}^{h} \\
& \quad+\left\langle\varsigma^{-1} \pi_{W}^{h}\left[\left|\vec{\theta}^{h}\right|^{-1}\left(\vec{Y}^{h} \cdot \vec{\omega}_{d}^{h}\right)\right] \vec{Q}_{\star}^{h} \vec{Y}^{h}, \vec{X}_{t, s}^{h}\right\rangle_{\Gamma^{h}}^{h}=-\left\langle\vec{Q}_{\star}^{h} \vec{X}_{t}^{h}, \vec{Q}_{\star}^{h} \vec{X}_{t}^{h}\right\rangle_{\Gamma^{h}}^{h} .
\end{aligned}
$$

The desired result (3.50a) then follows from (3.51) on recalling (3.9). 
Differentiating (3.49b) with respect to $t$ and then choosing $\vec{\eta}=\vec{Y}^{h} \in \underline{V}^{h}$, and combining this with (3.49a) with $\vec{\chi}=\vec{X}_{t}^{h} \in \underline{V}^{h}$ yields that

$$
\begin{aligned}
\frac{\mathrm{d}}{\mathrm{d} t}[ & \left.\frac{1}{2}\left\langle\varsigma^{-1} \vec{Q}_{\omega, \star}^{h} \vec{Y}^{h}, \vec{Y}^{h}\right\rangle_{\Gamma^{h}}^{h}+\langle\varsigma, \lambda\rangle_{\Gamma^{h}}^{h}\right] \\
& +\frac{1}{2}\left[\left\langle\varsigma^{-1}\left(\vec{Q}_{\omega, \star}^{h} \vec{Y}^{h}\right)_{t}, \vec{Y}^{h}\right\rangle_{\Gamma^{h}}^{h}-\left\langle\varsigma^{-1} \vec{Q}_{\omega, \star}^{h} \vec{Y}^{h}, \vec{Y}_{t}^{h}\right\rangle_{\Gamma^{h}}^{h}\right] \\
& +\left\langle\varsigma^{-1} \pi_{W}^{h}\left[\vec{Y}^{h} \cdot \vec{\omega}^{h}\right]\left[\left(\vec{Y}^{h} \cdot \vec{\omega}^{h}\right) \vec{X}_{s}^{h}-\left(\vec{Y}^{h}\right)^{\perp}\right], \vec{X}_{t, s}^{h}\right\rangle_{\Gamma^{h}}^{h}=-\left\langle\vec{Q}_{\omega, \star}^{h} \vec{X}_{t}^{h}, \vec{X}_{t}^{h}\right\rangle_{\Gamma^{h}}^{h} .
\end{aligned}
$$

The desired result (3.50b) then follows on noting that $\pi^{h}\left[\left(\vec{Q}_{\omega, \star}^{h} \vec{Y}^{h}\right)_{t} \vec{Y}^{h}-\right.$ $\left.\left(\vec{Q}_{\omega, \star}^{h}, \vec{Y}^{h}\right) \vec{Y}_{t}^{h}\right]=2 \pi_{W}^{h}\left[\left(\vec{Y}^{h} \cdot \vec{\omega}^{h}\right)\left(\vec{Y}^{h} \cdot \vec{\omega}_{t}^{h}\right)\right]$ and $(3.13)$.

Theorem 3.6. Let $N=2$. Let $\left(\mathcal{C}^{h}\right)$ hold and let $\left(\vec{X}^{h}(t), \vec{Y}^{h}(t)\right)_{t \in(0, T]}$ denote a solution to (3.48a), (3.48b) or to (3.49a), (3.49b). For a fixed $i \in\{1,2\}$ and a fixed time $t \in(0, T]$ let $\vec{a}_{j-\frac{1}{2}}^{h}:=\vec{X}_{i}^{h}\left(q_{j}^{i}, t\right)-\vec{X}_{i}^{h}\left(q_{j-1}^{i}, t\right), j=1 \rightarrow J_{i}$. Then it holds for $j=1 \rightarrow J_{i}-1$ that

$$
\left|\vec{a}_{j+\frac{1}{2}}^{h}\right|=\left|\vec{a}_{j-\frac{1}{2}}^{h}\right| \quad \text { if } \quad \vec{a}_{j+\frac{1}{2}}^{h} \nVdash \vec{a}_{j-\frac{1}{2}}^{h} .
$$

Proof. The proof is exactly the same as the proof of Theorem 3.4.

Remark 3.3. In practice it is most often of interest to consider length constraints for the elastic flows that we discuss in this paper. We can introduce stable semidiscrete finite element approximations for these flows, by mimicking on the discrete level the procedure in Remark 2.9 as follows. In the definitions of the discrete Lagrangians we replace the term $\langle\varsigma, \lambda\rangle_{\Gamma^{h}}$ by

$$
\sum_{i=1}^{N} \varsigma_{i} \lambda_{i}\left(\left|\Gamma_{i}^{h}\right|-l_{i}\right),
$$

where $l \in \mathbb{R}_{>0}^{N}$ are given lengths. We now consider $\lambda(t) \in \mathbb{R}^{N}$ as an unknown and a variation of the modified Lagrangian with respect to $\lambda$ gives the additional equations

$$
\left|\Gamma_{i}^{h}\right|=l_{i}, \quad i=1 \rightarrow N
$$

For example, as the length preserving approximation of the elastic flow in the case of two planar curves meeting at two $C^{1}$ junctions, we then obtain (3.49a), (3.49b) with the additional unknown $\lambda(t)$ and the additional constraints (3.54) for $l_{i}:=\left|\Gamma_{i}^{h}(0)\right|, i=1 \rightarrow N$. Similarly to Theorem 3.5 , it is a simple matter to show that this semidiscrete approximation fulfills $\frac{\mathrm{d}}{\mathrm{d} t}\left|\Gamma_{i}^{h}(t)\right|=0, i=1 \rightarrow N$, and $\frac{\mathrm{d}}{\mathrm{d} t} E_{0, \bar{\varkappa}}^{h}\left(\Gamma^{h}, \varsigma^{-1} \vec{Y}^{h} \cdot \vec{\omega}^{h}+\bar{\varkappa}\right)=-\left\langle\vec{Q}_{\omega, \star}^{h} \vec{X}_{t}^{h}, \vec{X}_{t}^{h}\right\rangle_{\Gamma^{h}}^{h} \leq 0$. 


\subsection{Nonlinear $C^{2}$ splines}

The derivation of the semidiscrete analogue of the weak formulation (2.67a), (2.67b) is similar to the derivation of $(3.48 \mathrm{a}),(3.48 \mathrm{~b})$ in $\$ 3.2$. The obtained approximation is as follows.

Given $\Gamma^{h}(0)=\vec{X}^{h}([0,1], 0)$, with $\vec{X}^{h}(0) \in \underline{V}_{s}^{h}$, for all $t \in(0, T]$ find $\Gamma^{h}(t)=$ $\vec{X}^{h}([0,1], t)$ with $\vec{X}_{t}^{h}(t) \in \underline{W}^{h}$, and $\vec{Y}^{h}(t) \in \underline{V}_{s}^{h}$ such that

$$
\begin{gathered}
\left\langle\vec{Q}_{\star}^{h} \vec{X}_{t}^{h}, \vec{\chi}\right\rangle_{\Gamma^{h}}^{h}-\left\langle\vec{\nabla}_{s} \vec{Y}^{h}, \vec{\nabla}_{s} \vec{\chi}\right\rangle_{\Gamma^{h}}-\frac{1}{2}\left\langle\left(\varsigma^{-1}\left|\vec{Q}_{\star}^{h} \vec{Y}^{h}\right|^{2}-2 \varsigma \lambda\right) \vec{X}_{s}^{h}, \vec{\chi}_{s}\right\rangle_{\Gamma^{h}}^{h} \\
+\left\langle\varsigma^{-1} \pi_{W}^{h}\left[\left|\vec{\theta}^{h}\right|^{-1}\left(\vec{Y}^{h} \cdot \vec{\omega}_{d}^{h}\right)\right] \vec{Q}_{\star}^{h} \vec{Y}^{h}, \vec{\chi}_{s}\right\rangle_{\Gamma^{h}}^{h}=0 \quad \forall \vec{\chi} \in \underline{W}^{h}, \\
\left\langle\varsigma^{-1} \vec{Q}_{\star}^{h} \vec{Y}^{h}, \vec{\eta}\right\rangle_{\Gamma^{h}}^{h}+\left\langle\vec{X}_{s}^{h}, \vec{\eta}_{s}\right\rangle_{\Gamma^{h}}=\vec{\zeta}_{N} \cdot \vec{\eta}_{N}(1)-\vec{\zeta}_{0} \cdot \vec{\eta}_{1}(0) \quad \forall \vec{\eta} \in \underline{V}_{s}^{h} .
\end{gathered}
$$

As before, we have that $\vec{\kappa}_{i}^{h}:=\varsigma_{i}^{-1} \pi^{h}\left[\vec{Q}_{\star, i}^{h} \vec{Y}_{i}^{h}\right]$ are the natural discrete approximations to $\vec{\varkappa}_{i}, i=1 \rightarrow N$,

Remark 3.4. Using the modified operator $\vec{Q}_{\star}^{h}$ in $(3.55 \mathrm{~b})$ is necessary in order to be able to prove existence and uniqueness of the discrete solution for the corresponding fully discrete approximation. However, it is also necessary in order to avoid enforcing equidistribution across all the curves, see Remark 3.2. The operator $\vec{Q}_{\star}^{h}$ and the projection $\pi_{W}^{h}$ then appear in (3.55a) for stability reasons. In fact, $(3.55 \mathrm{a})$ is the same as $(3.48 \mathrm{a})$ with the test space $\underline{V}^{h}$ replaced by $\underline{W}^{h}$.

Theorem 3.7. Let $\left(\mathcal{C}^{h}\right)$ hold and let $\left(\vec{X}^{h}(t), \vec{Y}^{h}(t)\right)_{t \in(0, T]}$ be a solution to (3.55a), (3.55b). Then we have that

$$
\frac{\mathrm{d}}{\mathrm{d} t} E_{\lambda}^{h}\left(\Gamma^{h}, \varsigma^{-1} \vec{Q}_{\star}^{h} \vec{Y}^{h}\right)=-\left\langle\vec{Q}_{\star}^{h} \vec{X}_{t}^{h}, \vec{Q}_{\star}^{h} \vec{X}_{t}^{h}\right\rangle_{\Gamma^{h}}^{h} \leq 0 .
$$

Proof. The proof is analogous to the proof of Theorem 3.5.

Theorem 3.8. Let $\left(\mathcal{C}^{h}\right)$ hold and let $\left(\vec{X}^{h}(t), \vec{Y}^{h}(t)\right)_{t \in(0, T]}$ denote a solution to (3.55a), (3.55b). For a fixed $i \in\{1, \ldots, N\}$ and a fixed time $t \in(0, T]$ let $\vec{a}_{j-\frac{1}{2}}^{h}:=$ $\vec{X}_{i}^{h}\left(q_{j}^{i}, t\right)-\vec{X}_{i}^{h}\left(q_{j-1}^{i}, t\right), j=1 \rightarrow J_{i}$. Then it holds for $j=1 \rightarrow J_{i}-1$ that

$$
\left|\vec{a}_{j+\frac{1}{2}}^{h}\right|=\left|\vec{a}_{j-\frac{1}{2}}^{h}\right| \quad \text { if } \quad \vec{a}_{j+\frac{1}{2}}^{h} \nVdash \vec{a}_{j-\frac{1}{2}}^{h} .
$$

Proof. The proof is exactly the same as the proof of Theorem 3.4.

\section{Fully discrete finite element approximation}

In this section we introduce fully discrete variants of the semidiscrete finite element approximations derived in Section 3 .

Let $0=t_{0}<t_{1}<\ldots<t_{M-1}<t_{M}=T$ be a partitioning of $[0, T]$ into possibly variable time steps $\tau_{m}:=t_{m+1}-t_{m}, m=0 \rightarrow M-1$. We set $\tau:=\max _{m=0 \rightarrow M-1} \tau_{m}$. Given $\Gamma^{0}=\vec{X}^{0}([0,1])$, our fully discrete approximation will define a sequence of polygonal curve networks $\Gamma^{m}, m=0 \rightarrow M$, where $\Gamma^{m}=\vec{X}^{m}([0,1])$ with $\vec{X}^{m} \in \underline{V}^{h}$. 
Similarly to (3.1), (3.4) and (3.7), we define $\langle u, v\rangle_{\Gamma^{m}}^{h}$ and introduce the definitions of $\vec{\theta}^{m}, \vec{\omega}_{d}^{m} \in \underline{S}^{h}$ and $\vec{Q}^{m}$, which are based on $\vec{X}^{m}$ in place of $\vec{X}^{h}(t)$. For the case $d=2$ we introduce, in a similar fashion, $\vec{\nu}^{m}:=-\left(\vec{X}_{s}^{m}\right)^{\perp}$, as well as $\vec{\omega}^{m} \in \underline{S}^{h}$ and $\vec{Q}_{\omega}^{m}$.

Similarly to $\left(\mathcal{C}^{h}\right)$, we make the following very weak assumptions.

$\left(\mathcal{C}^{m}\right)$ Let $(i) \vec{X}_{i}^{m}\left(q_{j}^{i}\right) \neq \vec{X}_{i}^{m}\left(q_{j+1}^{i}\right), j=0 \rightarrow J_{i}-1$, and $\vec{X}_{i}^{m}\left(q_{j-1}^{i}\right) \neq \vec{X}_{i}^{m}\left(q_{j+1}^{i}\right)$, $j=1 \rightarrow J_{i}-1$, for $i=1 \rightarrow N$.

\subsection{The $C^{0}$ case - attachment only}

Our fully discrete finite element schemes corresponding to (3.21a), (3.21b) and $(3.22 \mathrm{a}),(3.22 \mathrm{~b})$ are given as follows.

Find $\left(\vec{X}^{m+1}, \vec{Y}^{m+1}\right) \in \underline{V}^{h} \times \underline{W}^{h}$ such that

$$
\begin{aligned}
& \left\langle\vec{Q}_{\star}^{m} \frac{\vec{X}^{m+1}-\vec{X}^{m}}{\tau_{m}}, \vec{\chi}\right\rangle_{\Gamma^{m}}^{h}-\left\langle\vec{Y}_{s}^{m+1}, \vec{\chi}_{s}\right\rangle_{\Gamma^{m}}+\left\langle\left(\overrightarrow{I d}-\vec{P}^{m}\right) \vec{Y}_{s}^{m}, \vec{\chi}_{s}\right\rangle_{\Gamma^{m}} \\
& =\frac{1}{2}\left\langle\left(\varsigma^{-1}\left|\vec{Q}^{m} \vec{Y}^{m}\right|^{2}-2 \varsigma \lambda\right) \vec{X}_{s}^{m}, \vec{\chi}_{s}\right\rangle_{\Gamma^{m}}^{h}-\left\langle\varsigma^{-1}\left|\vec{\theta}^{m}\right|^{-1}\left(\vec{Y}^{m} \cdot \vec{\omega}_{d}^{m}\right) \vec{Q}^{m} \vec{Y}^{m}, \vec{\chi}_{s}\right\rangle_{\Gamma^{m}}^{h} \\
& \forall \vec{\chi} \in \underline{V}^{h},(4.1 \mathrm{a}) \\
& \left\langle\varsigma^{-1} \vec{Q}^{m} \vec{Y}^{m+1}, \vec{\eta}\right\rangle_{\Gamma^{m}}^{h}+\left\langle\vec{X}_{s}^{m+1}, \vec{\eta}_{s}\right\rangle_{\Gamma^{m}}=0 \quad \forall \vec{\eta} \in \underline{W}^{h} .
\end{aligned}
$$

where $\vec{P}^{m}=\overrightarrow{I d}-\vec{X}_{s}^{m} \otimes \vec{X}_{s}^{m}$.

Find $\left(\vec{X}^{m+1}, \vec{Y}^{m+1}\right) \in \underline{V}^{h} \times \underline{W}^{h}$ such that

$$
\begin{gathered}
\left\langle\vec{Q}_{\omega, \star}^{m} \frac{\vec{X}^{m+1}-\vec{X}^{m}}{\tau_{m}}, \vec{\chi}\right\rangle_{\Gamma^{m}}^{h}-\left\langle\vec{Y}_{s}^{m+1}, \vec{\chi}_{s}\right\rangle_{\Gamma^{m}}+\left\langle\left(\overrightarrow{I d}-\vec{P}^{m}\right) \vec{Y}_{s}^{m}, \vec{\chi}_{s}\right\rangle_{\Gamma^{m}} \\
=-\frac{1}{2}\left\langle\left(\varsigma^{-1}\left(\vec{Y}^{m} \cdot \vec{\omega}^{m}\right)^{2}+2 \varsigma \lambda\right) \vec{X}_{s}^{m}, \vec{\chi}_{s}\right\rangle_{\Gamma^{m}}^{h}+\left\langle\left(\varsigma^{-1} \vec{Y}^{m} \cdot \vec{\omega}^{m}+\vec{\varkappa}\left(\vec{Y}^{m}\right)^{\perp}, \vec{\chi}_{s}\right\rangle_{\Gamma^{m}}^{h}\right. \\
\forall \vec{\chi} \in \underline{V}^{h}, \quad(4.2 \mathrm{a}) \\
\left\langle\varsigma^{-1} \vec{Q}_{\omega}^{m} \vec{Y}^{m+1}, \vec{\eta}\right\rangle_{\Gamma^{m}}^{h}+\left\langle\vec{X}_{s}^{m+1}, \vec{\eta}_{s}\right\rangle_{\Gamma^{m}}=-\left\langle\vec{\varkappa}^{m}, \vec{\eta}\right\rangle_{\Gamma^{m}}^{h} \quad \forall \vec{\eta} \in{\underline{W^{h}}}^{h} .
\end{gathered}
$$

Here and throughout $\vec{Y}^{0}$ is a suitably chosen initial value. Of course, for the above we let $\vec{Y}^{0} \in \underline{W}^{h}$, while e.g. for the schemes in $\S 4.2$ we let $\vec{Y}^{0} \in \underline{V}^{h}$.

Remark 4.1. We note that the natural semi-implicit fully discrete approximation of (3.21a), (3.21b) has $-\left\langle\vec{\nabla}_{s} \vec{Y}^{m+1}, \vec{\nabla}_{s} \vec{\chi}\right\rangle_{\Gamma^{m}}$ instead of the last two terms on the left-hand side of (4.1a). However, then existence and uniqueness of the discrete solution $\left(\vec{X}^{m+1}, \vec{Y}^{m+1}\right) \in \underline{V}^{h} \times \underline{W}^{h}$ to this modified system is in general no longer guaranteed. In particular, existence and uniqueness can only be guaranteed if

$$
\left\langle\vec{\nabla}_{s} \vec{Y}, \vec{\nabla}_{s} \vec{Y}\right\rangle_{\Gamma^{m}}=0
$$

for $\vec{Y} \in \underline{W}^{h}$ implies that $\vec{Y}=\overrightarrow{0}$, which is only true if the curves $\Gamma_{i}^{m}, i=1 \rightarrow N$, have nowhere locally parallel segments. The same modification was employed by the authors for the fully discrete approximations in Ref. 7; see Remark 4.1 there. 
Theorem 4.1. Let the assumptions $\left(\mathcal{C}^{m}\right)$ hold. Then there exist unique solutions $\left(\vec{X}^{m+1}, \vec{Y}^{m+1}\right) \in \underline{V}^{h} \times \underline{W}^{h}$ to (4.1a), (4.1b) and (4.2a), (4.2b), respectively.

Proof. As (4.1a), (4.1b) is a linear system, existence follows from uniqueness. To investigate the latter, we consider the system: Find $(\vec{X}, \vec{Y}) \in \underline{V}^{h} \times \underline{W}^{h}$ such that

$$
\begin{aligned}
\tau_{m}^{-1}\left\langle\vec{Q}_{\star}^{m} \vec{X}, \vec{\chi}\right\rangle_{\Gamma^{m}}^{h}-\left\langle\vec{Y}_{s}, \vec{\chi}_{s}\right\rangle_{\Gamma^{m}}=0 & \forall \vec{\chi} \in \underline{V}^{h}, \\
\left\langle\varsigma^{-1} \vec{Q}_{\star}^{m} \vec{Y}, \vec{\eta}\right\rangle_{\Gamma^{m}}^{h}+\left\langle\vec{X}_{s}, \vec{\eta}_{s}\right\rangle_{\Gamma^{m}}=0 & \forall \vec{\eta} \in \underline{W^{h}} .
\end{aligned}
$$

Choosing $\vec{\chi}=\vec{X} \in \underline{V}^{h}$ in (4.3a) and $\vec{\eta}=\vec{Y} \in \underline{W}^{h}$ in (4.3b) yields that

$$
\tau_{m}^{-1}\left\langle\vec{Q}_{\star}^{m} \vec{X}, \vec{X}\right\rangle_{\Gamma^{m}}^{h}+\left\langle\varsigma^{-1} \vec{Q}_{\star}^{m} \vec{Y}, \vec{Y}\right\rangle_{\Gamma^{m}}^{h}=0
$$

It immediately follows from (4.4) that $\pi^{h}\left[\vec{Q}_{\star}^{m} \vec{X}\right]=\pi^{h}\left[\vec{Q}_{\star}^{m} \vec{Y}\right]=\overrightarrow{0}$, and so in particular that $\vec{X} \in \underline{W}^{h}$. Hence it follows from choosing $\vec{\chi}=\vec{Y} \in \underline{W}^{h} \subset \underline{V}^{h}$ in (4.3a) and $\vec{\eta}=\vec{X} \in \underline{W}^{h}$ in (4.3b) that $\left\langle\vec{Y}_{s}, \vec{Y}_{s}\right\rangle_{\Gamma^{h}}=\left\langle\vec{X}_{s}, \vec{X}_{s}\right\rangle_{\Gamma^{h}}=0$, which yields that $\vec{Y}=\vec{X}=\overrightarrow{0}$. Hence there exists a unique solution $\left(\vec{X}^{m+1}, \vec{Y}^{m+1}\right) \in \underline{V}^{h} \times \underline{W}^{h}$ to (4.1a), (4.1b). The proof for $(4.2 \mathrm{a}),(4.2 \mathrm{~b})$ is identical, on replacing $\vec{Q}_{\star}^{m}$ with $\vec{Q}_{\omega, \downarrow}^{m}$,

The above proof highlights the need for the introduction of the special projections $\vec{Q}_{\star}^{m}$ and $\vec{Q}_{\omega, \star}^{m}$, in order to be able to prove existence and uniqueness for a solution to (4.1a), (4.1b) and (4.2a), (4.2b), respectively. This is the reason, why the projections $\vec{Q}_{\star}^{h}$ and $\vec{Q}_{\omega, \star}^{h}$ have been employed in (3.21a) and (3.22a).

\subsection{The $C^{1}$ case - enforcing Young's law}

For $N \geq 3$, our fully discrete finite element scheme corresponding to (3.36a), (3.36b) is given by $(4.1 \mathrm{a}),(4.1 \mathrm{~b})$ with $\vec{Q}_{\star}^{m}$ replaced by $\vec{Q}^{m}$ and with $\underline{W}^{h}$ replaced by $\underline{V}^{h}$. Similarly, our fully discrete variant of (3.37a), (3.37b) is given by (4.2a), (4.2b) with $\vec{Q}_{\omega, \star}^{m}$ replaced by $\vec{Q}_{\omega}^{m}$ and with $\underline{W}^{h}$ replaced by $\underline{V}^{h}$. Existence of a unique solution to these schemes then follows as in Theorem 4.1.

For $N=2$, the fully discrete finite element schemes corresponding to (3.48a), (3.48b) and (3.49a), (3.49b) are given as follows.

Find $\left(\vec{X}^{m+1}, \vec{Y}^{m+1}\right) \in \underline{V}^{h} \times \underline{V}^{h}$ such that

$$
\begin{gathered}
\left\langle\vec{Q}_{\star}^{m} \frac{\vec{X}^{m+1}-\vec{X}^{m}}{\tau_{m}}, \vec{\chi}\right\rangle_{\Gamma^{m}}^{h}-\left\langle\vec{Y}_{s}^{m+1}, \vec{\chi}_{s}\right\rangle_{\Gamma^{m}}+\left\langle\left(\overrightarrow{I d}-\vec{P}^{m}\right) \vec{Y}_{s}^{m}, \vec{\chi}_{s}\right\rangle_{\Gamma^{m}} \\
=\frac{1}{2}\left\langle\left(\varsigma^{-1}\left|\vec{Q}_{\star}^{m} \vec{Y}^{m}\right|^{2}-2 \varsigma \lambda\right) \vec{X}_{s}^{m}, \vec{\chi}_{s}\right\rangle_{\Gamma^{m}}^{h} \\
-\left\langle\varsigma^{-1} \pi_{W}^{h}\left[\left|\vec{\theta}^{m}\right|^{-1}\left(\vec{Y}^{m} \cdot \vec{\omega}_{d}^{m}\right)\right] \vec{Q}_{\star}^{m} \vec{Y}^{m}, \vec{\chi}_{s}\right\rangle_{\Gamma^{m}}^{h} \quad \forall \vec{\chi} \in \underline{V}^{h}, \\
\left\langle\varsigma^{-1} \vec{Q}_{\star}^{m} \vec{Y}^{m+1}, \vec{\eta}\right\rangle_{\Gamma^{m}}^{h}+\left\langle\vec{X}_{s}^{m+1}, \vec{\eta}_{s}\right\rangle_{\Gamma^{m}}=0 \quad \forall \vec{\eta} \in \underline{V}^{h} .
\end{gathered}
$$


Find $\left(\vec{X}^{m+1}, \vec{Y}^{m+1}\right) \in \underline{V}^{h} \times \underline{V}^{h}$ such that

$$
\begin{aligned}
&\left\langle\vec{Q}_{\omega, \star}^{m} \frac{\vec{X}^{m+1}-\vec{X}^{m}}{\tau_{m}},\right.\vec{\chi}\rangle_{\Gamma^{m}}^{h}-\left\langle\vec{Y}_{s}^{m+1}, \vec{\chi}_{s}\right\rangle_{\Gamma^{m}}+\left\langle\left(\overrightarrow{I d}-\vec{P}^{m}\right) \vec{Y}_{s}^{m}, \vec{\chi}_{s}\right\rangle_{\Gamma^{m}} \\
&=-\frac{1}{2}\left\langle\left(2 \varsigma^{-1} \pi_{W}^{h}\left[\left(\vec{Y}^{m} \cdot \vec{\omega}^{m}\right)^{2}\right]-\varsigma^{-1}\left(\vec{Q}_{\omega, \star}^{m} \vec{Y}^{m}\right) . \vec{Y}^{m}+2 \varsigma \lambda\right) \vec{X}_{s}^{m}, \vec{\chi}_{s}\right\rangle_{\Gamma^{m}}^{h} \\
&+\left\langle\left(\varsigma^{-1} \pi_{W}^{h}\left[\vec{Y}^{m} \cdot \vec{\omega}^{m}\right]+\bar{\varkappa}\right)\left(\vec{Y}^{m}\right)^{\perp}, \vec{\chi}_{s}\right\rangle_{\Gamma^{m}}^{h} \quad \forall \vec{\chi} \in \underline{V}^{h}, \\
&\left\langle\varsigma^{-1} \vec{Q}_{\omega, \star}^{m} \vec{Y}^{m+1}, \vec{\eta}\right\rangle_{\Gamma^{m}}^{h}+\left\langle\vec{X}_{s}^{m+1}, \vec{\eta}_{s}\right\rangle_{\Gamma^{m}}=-\left\langle\vec{\varkappa}^{m}, \vec{\eta}\right\rangle_{\Gamma^{m}}^{h} \quad \forall \vec{\eta} \in \underline{V}^{h} .
\end{aligned}
$$

Theorem 4.2. Let the assumptions $\left(\mathcal{C}^{m}\right)$ hold. Then there exist unique solutions $\left(\vec{X}^{m+1}, \vec{Y}^{m+1}\right) \in \underline{V}^{h} \times \underline{V}^{h}$ to (4.5a), (4.5b) and (4.6a), (4.6b), respectively.

Proof. The proof is analogous to the proof of Theorem 4.1.

Remark 4.2. We remark that (4.5a), (4.5b) with the trial space for $\vec{Y}^{m}$ replaced by $\underline{W}^{h}$ and the test space for $(4.5 \mathrm{~b})$ replaced by $\underline{W}^{h}$ is exactly the same as (4.1a), (4.1b); and similarly for the schemes (4.6a), (4.6b) and (4.2a), (4.2b). The reason is that the modifications in (4.5a) compared to (4.1a), (4.1b) have no effect when $\vec{Y}^{m} \in \underline{W}^{h}$. But this point of view will make it easier to present the solution methods in Section 5.

Remark 4.3. For any of our fully discrete schemes, we can approximate the corresponding length preserving flow by replacing the fixed given $\lambda \in \mathbb{R}^{N}$ by a $\lambda^{m+1} \in \mathbb{R}^{N}$, where these unknowns are chosen so that $\left|\Gamma_{i}^{m+1}\right|=\left|\Gamma_{i}^{0}\right|, i=1 \rightarrow N$. Then we obtain fully discrete versions of the semidiscrete schemes discussed in Remark 3.3. In each case, the fully discrete approximation then leads to a nonlinear system of equations at each time level, which can be solved by a root finding method in terms of $\lambda^{m+1}$, e.g. by an $N$-dimensional generalization of the secant method.

\subsection{Nonlinear splines}

Given $\vec{X}^{0} \in \underline{V}_{s}^{h}$ for $m=0 \rightarrow M-1$ find $\left(\delta \vec{X}^{m+1}, \vec{Y}^{m+1}\right) \in \underline{W}^{h} \times \underline{V}_{s}^{h}$, with $\vec{X}^{m+1}=\vec{X}^{m}+\delta \vec{X}^{m+1}$, such that

$$
\begin{aligned}
& \left\langle\vec{Q}_{\star}^{m} \frac{\delta \vec{X}^{m+1}}{\tau_{m}}, \vec{\chi}\right\rangle_{\Gamma^{m}}^{h}-\left\langle\vec{Y}_{s}^{m+1}, \vec{\chi}_{s}\right\rangle_{\Gamma^{m}}+\left\langle\left(\overrightarrow{I d}-\vec{P}^{m}\right) \vec{Y}_{s}^{m}, \vec{\chi}_{s}\right\rangle_{\Gamma^{m}} \\
& =\frac{1}{2}\left\langle\left(\varsigma^{-1}\left|\vec{Q}_{\star}^{m} \vec{Y}^{m}\right|^{2}-2 \varsigma \lambda\right) \vec{X}_{s}^{m}, \vec{\chi}_{s}\right\rangle_{\Gamma^{m}}^{h} \\
& -\left\langle\varsigma^{-1} \pi_{W}^{h}\left[\left|\vec{\theta}^{m}\right|^{-1}\left(\vec{Y}^{m} \cdot \vec{\omega}_{d}^{m}\right)\right] \vec{Q}_{\star}^{m} \vec{Y}^{m}, \vec{\chi}_{s}\right\rangle_{\Gamma^{m}}^{h} \quad \forall \vec{\chi} \in \underline{W}^{h}, \\
& \left\langle\varsigma^{-1} \vec{Q}_{\star}^{m} \vec{Y}^{m+1}, \vec{\eta}\right\rangle_{\Gamma^{m}}^{h}+\left\langle\vec{X}_{s}^{m+1}, \vec{\eta}_{s}\right\rangle_{\Gamma^{m}}=\vec{\zeta}_{N} \cdot \vec{\eta}_{N}(1)-\vec{\zeta}_{0} \cdot \vec{\eta}_{1}(0) \quad \forall \vec{\eta} \in \underline{V}_{s}^{h} .
\end{aligned}
$$

Theorem 4.3. Let the assumptions $\left(\mathcal{C}^{m}\right)$ hold. Then there exists a unique solution $\left(\delta \vec{X}^{m+1}, \vec{Y}^{m+1}\right) \in \underline{W}^{h} \times \underline{V}_{s}^{h}$ to (4.7a), (4.7b).

Proof. The proof is analogous to the proof of Theorem 4.1. 


\section{Solution of the algebraic equations}

In line with previous work by the authors on the evolution of curve networks and surface clusters, ${ }^{2,1,5}$ in order to solve the fully discrete approximations from Section 4 in practice, it is easiest to formulate the linear systems of equations over the unconstrained finite element space $\underline{S}^{h}$. That means that the system matrices and right hand sides can be assembled independently for each curve $\Gamma_{i}^{m}, i=1 \rightarrow N$. The effect of the test and trial spaces $\underline{V}^{h}, \underline{W}^{h}$ and $\underline{V}_{s}^{h}$ can then be incorporated with the help of orthogonal projections from $\underline{S}^{h}$ onto these subspaces.

For each $i \in\{1, \ldots, N\}$, we introduce also the diagonal matrices $\overrightarrow{\mathcal{M}}_{(\star)}^{i}, \overrightarrow{\mathcal{M}}_{\omega(, \star)}^{i} \in\left(\mathbb{R}^{d \times d}\right)^{\left(J_{i}+1\right) \times\left(J_{i}+1\right)}$, and the stiffness matrices $A^{i} \in \mathbb{R}^{\left(J_{i}+1\right) \times\left(J_{i}+1\right)}$, $\vec{A}^{i}, \vec{A}_{Q_{(\star)}}^{i}, \vec{A}_{\omega(, \star)}^{i}, \overrightarrow{\mathcal{A}}^{i} \in\left(\mathbb{R}^{d \times d}\right)^{\left(J_{i}+1\right) \times\left(J_{i}+1\right)}$ with entries

$$
\begin{aligned}
{\left[\overrightarrow{\mathcal{M}}_{(\star)}^{i}\right]_{k l} } & :=\left\langle\chi_{k}^{i}, \chi_{l}^{i} \vec{Q}_{(\star,) i}^{m}\right\rangle_{\Gamma_{i}^{m}}^{h}, \quad\left[\overrightarrow{\mathcal{M}}_{\omega(, \star \star}^{i}\right]_{k l}:=\left\langle\chi_{k}^{i}, \chi_{l}^{i} \vec{Q}_{\omega(, \star), i}^{m}\right\rangle_{\Gamma_{i}^{m}}^{h}, \\
A_{k l}^{i} & :=\left\langle\left[\chi_{k}^{i}\right]_{s},\left[\chi_{l}^{i}\right]_{s}\right\rangle_{\Gamma_{i}^{m}}, \quad \vec{A}_{k l}^{i}:=A_{k l}^{i} \overrightarrow{I d}, \\
{\left[\vec{A}_{Q_{(\star)}}^{i}\right]_{k l} } & :=\frac{1}{2}\left\langle s_{i}^{-1}\left|\vec{Q}_{(\star,) i}^{m} \vec{Y}_{i}^{m}\right|^{2}\left[\chi_{k}^{i}\right]_{s},\left[\chi_{l}^{i}\right]_{s}\right\rangle_{\Gamma_{i}^{m}}^{h} \overrightarrow{I d}, \\
{\left[\vec{A}_{\omega}^{i}\right]_{k l} } & :=\frac{1}{2}\left\langle s_{i}^{-1}\left(\vec{Y}_{i}^{m} \cdot \vec{\omega}_{i}^{m}\right)^{2}\left[\chi_{k}^{i}\right]_{s},\left[\chi_{l}^{i}\right]_{s}\right\rangle_{\Gamma_{i}^{m}}^{h} \overrightarrow{I d}, \\
{\left[\vec{A}_{\omega, \star}^{i}\right]_{k l} } & :=\frac{1}{2}\left\langle s_{i}^{-1}\left(2 \pi_{W}^{h}\left[\left(\vec{Y}_{i}^{m} \cdot \vec{\omega}_{i}^{m}\right)^{2}\right]-\left(\vec{Q}_{\omega, \star, i}^{m} \vec{Y}_{i}^{m}\right) \cdot \vec{Y}_{i}^{m}\right)\left[\chi_{k}^{i}\right]_{s},\left[\chi_{l}^{i}\right]_{s}\right\rangle_{\Gamma_{i}^{m}}^{h} \overrightarrow{I d}, \\
\overrightarrow{\mathcal{A}}_{k l}^{i} & :=\left\langle\left[\chi_{k}^{i}\right]_{s},\left[\chi_{l}^{i}\right]_{s} \vec{P}_{i}^{m}\right\rangle_{\Gamma_{i}^{m}},
\end{aligned}
$$

where $\overrightarrow{I d}$ is the identity matrix in $\mathbb{R}^{d \times d}$; and where $\cdot_{(\star)}$ denotes an expression with or without the subscript $\star$.

Let $J:=\sum_{i=1}^{N}\left(J_{i}+1\right)$. We define the orthogonal projections

$$
\begin{gathered}
\overrightarrow{\mathcal{P}}_{W}:\left(\mathbb{R}^{d}\right)^{J} \rightarrow \underline{\mathbb{W}}:=\left\{\left(\vec{z}^{1}, \ldots, \vec{z}^{N}\right) \in\left(\mathbb{R}^{d}\right)^{J}:\left[\vec{z}^{i}\right]_{1}=\left[\vec{z}^{i}\right]_{J_{i}+1}=\overrightarrow{0}, i=1 \rightarrow N\right\}, \\
\overrightarrow{\mathcal{P}}_{V}:\left(\mathbb{R}^{d}\right)^{J} \rightarrow \underline{\mathbb{V}}:=\left\{\left(\vec{z}^{1}, \ldots, \vec{z}^{N}\right) \in\left(\mathbb{R}^{d}\right)^{J}:\left[\vec{z}^{1}\right]_{1}=\left[\vec{z}^{i}\right]_{1},\left[\vec{z}^{1}\right]_{J_{1}+1}=\left[\vec{z}^{i}\right]_{J_{i}+1},\right. \\
i=2 \rightarrow N\}, \\
\overrightarrow{\mathcal{P}}_{V_{s}}:\left(\mathbb{R}^{d}\right)^{J} \rightarrow \underline{\mathbb{V}}_{s}:=\left\{\left(\vec{z}^{1}, \ldots, \vec{z}^{N}\right) \in\left(\mathbb{R}^{d}\right)^{J}:\left[\vec{z}^{i}\right]_{J_{i}+1}=\left[\vec{z}^{i+1}\right]_{1}, i=1 \rightarrow N-1\right\}
\end{gathered}
$$

onto the Euclidean spaces associated with $\underline{W}^{h}, \underline{V}^{h}$ and $\underline{V}_{s}^{h}$, respectively. These projections will be crucial for the construction of the linear systems for the unknown coefficient vectors in $\left(\mathbb{R}^{d}\right)^{J} \times\left(\mathbb{R}^{d}\right)^{J}$ that represent the solutions to our schemes in Section 4.

Finally, let the diagonal block matrices $\overrightarrow{\mathcal{M}}_{(\star)}, \overrightarrow{\mathcal{M}}_{\omega(, \star)}, \vec{A}, \vec{A}_{Q_{(\star)}}, \vec{A}_{\omega(, \star)}, \overrightarrow{\mathcal{A}} \in$ $\left(\mathbb{R}^{d \times d}\right)^{J \times J}$ be defined by

$$
\begin{aligned}
\overrightarrow{\mathcal{M}}_{(\star)} & :=\operatorname{diag}\left(\overrightarrow{\mathcal{M}}_{(\star)}^{1}, \ldots, \overrightarrow{\mathcal{M}}_{(\star)}^{N}\right), \quad \overrightarrow{\mathcal{M}}_{\omega(, \star)}:=\operatorname{diag}\left(\overrightarrow{\mathcal{M}}_{\omega(, \star)}^{1}, \ldots, \overrightarrow{\mathcal{M}}_{\omega(, \star)}^{N}\right), \\
\vec{A} & :=\operatorname{diag}\left(\vec{A}^{1}, \ldots, \vec{A}^{N}\right), \quad \vec{A}_{Q_{(\star)}}:=\operatorname{diag}\left(\vec{A}_{Q_{(\star)}}^{1}, \ldots, \vec{A}_{Q_{(\star)}}^{N}\right) \\
\vec{A}_{\omega(, \star)} & :=\operatorname{diag}\left(\vec{A}_{\omega(, \star)}^{1}, \ldots, \vec{A}_{\omega(, \star)}^{N}\right), \quad \overrightarrow{\mathcal{A}}:=\operatorname{diag}\left(\overrightarrow{\mathcal{A}}^{1}, \ldots, \overrightarrow{\mathcal{A}}^{N}\right)
\end{aligned}
$$




\subsection{The $C^{0}$ case - attachment only}

On recalling Remark 4.2, the linear system for the approximation (4.1a), (4.1b) can be formulated as: Find $\left(\vec{Y}^{m+1}, \delta \vec{X}^{m+1}\right) \in \underline{\mathbb{W}} \times \underline{\mathbb{V}}$, where here and throughout $\vec{X}^{m+1}=\vec{X}^{m}+\delta \vec{X}^{m+1}$, such that

$$
\begin{aligned}
& \left(\begin{array}{cc}
\overrightarrow{\mathcal{P}}_{V} \vec{A} \overrightarrow{\mathcal{P}}_{W} & -\frac{1}{\tau_{m}} \overrightarrow{\mathcal{P}}_{V} \overrightarrow{\mathcal{M}}_{\star} \overrightarrow{\mathcal{P}}_{V} \\
\overrightarrow{\mathcal{P}}_{W} \varsigma^{-1} \overrightarrow{\mathcal{M}}_{\star} \overrightarrow{\mathcal{P}}_{W} & \overrightarrow{\mathcal{P}}_{W} \vec{A} \overrightarrow{\mathcal{P}}_{V}
\end{array}\right)\left(\begin{array}{c}
\vec{Y}^{m+1} \\
\delta \vec{X}^{m+1}
\end{array}\right)=\left(\begin{array}{c}
\overrightarrow{\mathcal{P}}_{V} \vec{g}_{\star} \\
-\overrightarrow{\mathcal{P}}_{W}\left(\vec{A} \vec{X}^{m}\right)
\end{array}\right), \\
& \text { where } \vec{g}_{(\star)}:=(\vec{A}-\overrightarrow{\mathcal{A}}) \vec{Y}^{m}+\left(\varsigma \lambda \vec{A}-\vec{A}_{Q_{(\star)}}\right) \vec{X}^{m}+\vec{f}_{(\star)},
\end{aligned}
$$

where $\vec{f}_{(\star)}=\left(\vec{f}_{(\star)}^{1}, \ldots, \vec{f}_{(\star)}^{N}\right) \in\left(\mathbb{R}^{d}\right)^{J}$ with $\left[\vec{f}_{\star}^{i}\right]_{j+1}=\left\langle\pi_{W}^{h}\left[\left|\vec{\theta}_{i}^{m}\right|^{-1}\left(\vec{Y}_{i}^{m} \cdot \vec{\omega}_{d, i}^{m}\right)\right] \vec{Q}_{\star, i}^{m} \vec{Y}_{i}^{m}\right.$, $\left.\left[\chi_{j}^{i}\right]_{s}\right\rangle_{\Gamma_{i}^{m}}^{h}$, and, for later use, $\left[\overrightarrow{f^{i}}\right]_{j+1}=\left\langle\left|\vec{\theta}_{i}^{m}\right|^{-1}\left(\vec{Y}_{i}^{m} \cdot \vec{\omega}_{d, i}^{m}\right) \vec{Q}_{i}^{m} \vec{Y}_{i}^{m},\left[\chi_{j}^{i}\right]_{s}\right\rangle_{\Gamma_{i}^{m}}^{h}$, in each case for $j=0 \rightarrow J_{i}, i=1 \rightarrow N$. Here and throughout we use $\varsigma \lambda \vec{A}$ as a shorthand for the block diagonal matrix $\operatorname{diag}\left(\varsigma_{1} \lambda_{1} \vec{A}^{1}, \ldots, \varsigma_{N} \lambda_{N} \vec{A}^{N}\right)$; and similarly for $\varsigma^{-1} \overrightarrow{\mathcal{M}}_{\star}$.

Similarly, the linear system for $(4.2 \mathrm{a}),(4.2 \mathrm{~b})$ can be formulated as: Find $\left(\vec{Y}^{m+1}, \delta \vec{X}^{m+1}\right) \in \underline{\mathbb{W}} \times \underline{\mathbb{V}}$ such that

$$
\left(\begin{array}{ccc}
\overrightarrow{\mathcal{P}}_{V} \vec{A} \overrightarrow{\mathcal{P}}_{W} & -\frac{1}{\tau_{m}} \overrightarrow{\mathcal{P}}_{V} \overrightarrow{\mathcal{M}}_{\omega, \star} \overrightarrow{\mathcal{P}}_{V} \\
\overrightarrow{\mathcal{P}}_{W} \varsigma^{-1} \overrightarrow{\mathcal{M}}_{\omega, \star} \overrightarrow{\mathcal{P}}_{W} & \overrightarrow{\mathcal{P}}_{W} \vec{A}_{V}
\end{array}\right)\left(\begin{array}{c}
\vec{Y}^{m+1} \\
\delta \vec{X}^{m+1}
\end{array}\right)=\left(\begin{array}{c}
\overrightarrow{\mathcal{P}}_{V} \vec{h}_{\star} \\
-\overrightarrow{\mathcal{P}}_{W}\left(\vec{A} \vec{X}^{m}+\vec{c}\right)
\end{array}\right)
$$

where $\quad \vec{h}_{(\star)}:=(\vec{A}-\overrightarrow{\mathcal{A}}) \vec{Y}^{m}+\left(\varsigma \lambda \vec{A}+\vec{A}_{\omega(, \star)}\right) \vec{X}^{m}-\vec{b}_{(\star)}$,

where $\vec{b}_{(\star)}=\left(\vec{b}_{(\star)}^{1}, \ldots, \vec{b}_{(\star)}^{N}\right), \vec{c}=\left(\vec{c}^{1}, \ldots, \vec{c}^{N}\right) \in\left(\mathbb{R}^{d}\right)^{J}$ with $\left[\vec{b}_{\star}^{i}\right]_{j+1}=$ $\left\langle\varsigma^{-1} \pi_{W}^{h}\left[\left(\vec{Y}_{i}^{m} \cdot \vec{\omega}_{i}^{m}\right]+\bar{\varkappa}_{i}\right)\left(\vec{Y}_{i}^{m}\right)^{\perp},\left[\chi_{j}^{i}\right]_{s}\right\rangle_{\Gamma_{i}^{m}}^{h}$, and, for later use, $\left[\vec{b}^{i}\right]_{j+1}=$ $\left\langle\varsigma^{-1}\left(\vec{Y}_{i}^{m} \cdot \vec{\omega}_{i}^{m}+\bar{\varkappa}_{i}\right)\left(\vec{Y}_{i}^{m}\right)^{\perp},\left[\chi_{j}^{i}\right]_{s}\right\rangle_{\Gamma_{i}^{m}}^{h}$, and $\left[\vec{c}^{i}\right]_{j+1}=\bar{\varkappa}_{i}\left\langle\vec{\omega}_{i}^{m}, \chi_{j}^{i}\right\rangle_{\Gamma_{i}^{m}}^{h}$, in each case for $j=0 \rightarrow J_{i}, i=1 \rightarrow N$.

\subsection{The $C^{1}$ case - enforcing Young's law}

For $N \geq 3$, the linear systems for our fully discrete finite element schemes corresponding to (3.36a), (3.36b) and (3.37a), (3.37b) are given by (5.3a), (5.3b) and (5.4a), (5.4b), respectively, with $\overrightarrow{\mathcal{P}}_{W}$ replaced by $\overrightarrow{\mathcal{P}}_{V}$ and with $\overrightarrow{\mathcal{M}}_{\star}, \vec{g}_{\star}, \vec{h}_{\star}$ replaced by $\overrightarrow{\mathcal{M}}, \vec{g}, \vec{h}$.

For $N=2$, the linear systems for our fully discrete finite element schemes (4.5a), (4.5b) and (4.6a), (4.6b) are given by (5.3a), (5.3b) and (5.4a), (5.4b), respectively, with $\overrightarrow{\mathcal{P}}_{W}$ replaced by $\overrightarrow{\mathcal{P}}_{V}$.

\subsection{Nonlinear $C^{2}$ splines}

The linear system for our fully discrete finite element scheme (4.7a), (4.7b) is given by $(5.3 \mathrm{a}),(5.3 \mathrm{~b})$ with $\overrightarrow{\mathcal{P}}_{V}$ replaced by $\overrightarrow{\mathcal{P}}_{W}, \overrightarrow{\mathcal{P}}_{W}$ replaced by $\overrightarrow{\mathcal{P}}_{V_{s}}$ and with the additional contribution $\vec{d} \in\left(\mathbb{R}^{d}\right)^{J}$ added to the right hand side of the second equation in (5.3a), where $\vec{d}_{1}=-\vec{\zeta}_{0}, \vec{d}_{J}=\vec{\zeta}_{N}$ and $\vec{d}_{j}=\overrightarrow{0}$ for $j=2 \rightarrow J-1$. 


\subsection{Solution methods}

All of the stated linear systems in this section can be written as

$$
\begin{aligned}
& \left(\begin{array}{cc}
\overrightarrow{\mathcal{P}}_{X} & 0 \\
0 & \overrightarrow{\mathcal{P}}_{Y}
\end{array}\right)\left(\begin{array}{cc}
\vec{A}_{Y} & -\frac{1}{\tau_{m}} \vec{M} \\
\varsigma^{-1} \vec{M} & \vec{A}_{X}
\end{array}\right)\left(\begin{array}{cc}
\overrightarrow{\mathcal{P}}_{Y} & 0 \\
0 & \overrightarrow{\mathcal{P}}_{X}
\end{array}\right)\left(\begin{array}{c}
\vec{Y}^{m+1} \\
\delta \vec{X}^{m+1}
\end{array}\right)=\left(\begin{array}{cc}
\overrightarrow{\mathcal{P}}_{X} & 0 \\
0 & \overrightarrow{\mathcal{P}}_{Y}
\end{array}\right)\left(\begin{array}{l}
\vec{f}_{X} \\
\vec{f}_{Y}
\end{array}\right), \\
& \overrightarrow{\mathcal{P}}_{Y} \vec{Y}^{m+1}=\vec{Y}^{m+1}, \quad \overrightarrow{\mathcal{P}}_{X} \delta \vec{X}^{m+1}=\delta \vec{X}^{m+1},
\end{aligned}
$$

where, apart from the obvious block matrices and right hand sides, $\overrightarrow{\mathcal{P}}_{X}$ and $\overrightarrow{\mathcal{P}}_{Y}$ are projections onto the Euclidean solution spaces for $\delta \vec{X}^{m+1}$ and $\vec{Y}^{m+1}$, respectively.

A possible solution method for (5.5a) would be to employ a preconditioned BiCGSTAB iterative solver together with the preconditioner

$$
\left(\begin{array}{cc}
\overrightarrow{\mathcal{P}}_{Y} & 0 \\
0 & \overrightarrow{\mathcal{P}}_{X}
\end{array}\right)\left(\begin{array}{cc}
\vec{A}_{Y} & -\frac{1}{\tau_{m}} \vec{M} \\
\varsigma^{-1} \vec{M} & \vec{A}_{X}
\end{array}\right)^{-1}\left(\begin{array}{cc}
\overrightarrow{\mathcal{P}}_{X} & 0 \\
0 & \overrightarrow{\mathcal{P}}_{Y}
\end{array}\right)
$$

if this is well-defined, and otherwise use (5.6) with $\vec{A}_{X}$ and $\vec{A}_{Y}$ replaced by $\operatorname{diag}\left(\vec{A}_{X}\right)$ and $\operatorname{diag}\left(\vec{A}_{Y}\right)$, respectively. The inverse in (5.6) could be conveniently computed with the help of a sparse factorization solver.

However, we prefer to solve $(5.5 \mathrm{a}),(5.5 \mathrm{~b})$ with a direct method as follows. The recently made available sparse $Q R$ factorization package SuiteSparseQR, ${ }^{9}$ allows the computation of least squares solutions to both overdetermined and underdetermined linear systems. From the theoretical results in Section 4 we know that there exists a solution $\left(\vec{Y}^{m+1}, \delta \vec{X}^{m+1}\right)$ to $(5.5 \mathrm{a})$ in the subspace $\left(\overrightarrow{\mathcal{P}}_{Y}\left(\mathbb{R}^{d}\right)^{J}\right) \times\left(\overrightarrow{\mathcal{P}}_{X}\left(\mathbb{R}^{d}\right)^{J}\right)$ of $\left(\mathbb{R}^{d}\right)^{J} \times\left(\mathbb{R}^{d}\right)^{J}$. Hence any least squares solution to $\left(5.5\right.$ a) over $\left(\mathbb{R}^{d}\right)^{J} \times\left(\mathbb{R}^{d}\right)^{J}$ will in fact solve (5.5a). Moreover, on obtaining one such solution $\left(\vec{Y}_{u}, \delta \vec{X}_{u}\right) \in$ $\left(\mathbb{R}^{d}\right)^{J} \times\left(\mathbb{R}^{d}\right)^{J}$ to $(5.5 \mathrm{a})$, the solution to $(5.5 \mathrm{a}),(5.5 \mathrm{~b})$ is given by $\left(\vec{Y}^{m+1}, \delta \vec{X}^{m+1}\right)=$ $\left(\overrightarrow{\mathcal{P}}_{Y} \vec{Y}_{u}, \overrightarrow{\mathcal{P}}_{X} \delta \vec{X}_{u}\right)$. In practice, we use the package SuiteSparseQR to find a $\left(\vec{Y}_{u}, \delta \vec{X}_{u}\right)$.

Remark 5.1. The global projections $\overrightarrow{\mathcal{P}}_{X}$ and $\overrightarrow{\mathcal{P}}_{Y}$ in (5.5a) acting on vectors $\vec{z} \in\left(\mathbb{R}^{d}\right)^{J}$ can always be decomposed into independent local projections acting on (possible combinations of) the $2 N$ coordinates $\left[\vec{z}^{i}\right]_{j+1}, j \in\left\{0, J_{i}\right\}, i=1 \rightarrow N$, of $\vec{z}$ only. Throughout, these local projections, denoted by $\overrightarrow{\mathrm{P}}_{X}, \overrightarrow{\mathrm{P}}_{Y}$, fall into only four possible categories. They are

(i) $C^{0}$ - attachment only: $\overrightarrow{\mathrm{P}}_{X}=\overrightarrow{\mathrm{P}}_{V}, \overrightarrow{\mathrm{P}}_{Y}=\overrightarrow{\mathrm{P}}_{W}$.

(ii) $C^{1}$ - enforcing Young's law: $\overrightarrow{\mathrm{P}}_{X}=\overrightarrow{\mathrm{P}}_{V}, \overrightarrow{\mathrm{P}}_{Y}=\overrightarrow{\mathrm{P}}_{V}$.

(iii) Nonlinear $C^{2}$ spline: $\overrightarrow{\mathrm{P}}_{X}=\overrightarrow{\mathrm{P}}_{W}, \overrightarrow{\mathrm{P}}_{Y}=\overrightarrow{\mathrm{P}}_{V}$.

(iv) Boundary point with clamped boundary conditions: $\overrightarrow{\mathrm{P}}_{X}=\overrightarrow{\mathrm{P}}_{W}, \overrightarrow{\mathrm{P}}_{Y}=\overrightarrow{I d}$.

Here we note that $\overrightarrow{\mathrm{P}}_{W}$ is simply the zero map/matrix.

Any of the global projections $\overrightarrow{\mathcal{P}}_{X}$ and $\overrightarrow{\mathcal{P}}_{Y}$ considered in this paper can then be constructed from the four types of local projections $\overrightarrow{\mathrm{P}}_{X}$ and $\overrightarrow{\mathrm{P}}_{Y}$ described above. Moreover, it is now easily possible to use the description (5.5a), (5.5b) in order to formulate the linear systems for fully discrete finite element approximations of the elastic flow for more general types of curve networks, as indicated in $§ 2.4$. 
Remark 5.2. For the fully discrete length preserving approximations discussed in Remark 4.3, we need to find values $\lambda^{m+1} \in \mathbb{R}^{N}$ such that $F\left(\lambda^{m+1}\right)=0$, where $F$ : $\mathbb{R}^{N} \rightarrow \mathbb{R}^{N}$ for an arbitrary $\lambda \in \mathbb{R}^{N}$ is defined by $F(\lambda)=\left(\left|\Gamma_{1}^{m+1}\right|-\left|\Gamma_{1}^{0}\right|, \ldots,\left|\Gamma_{N}^{m+1}\right|-\right.$ $\left.\left|\Gamma_{N}^{0}\right|\right)$, where $\Gamma^{m+1}=\vec{X}^{m+1}([0,1])$ is obtained from the appropriate approximation in Section 4 with this value of $\lambda$. Hence a function evaluation of $F$ corresponds to the solution of a system of the form (5.5a), (5.5b). As we use the sparse factorization package SuiteSparseQR to solve (5.5a), (5.5b) in practice, repeated evaluations of $F$ are cheap. Therefore we employ an $N$-dimensional generalization of the secant method to solve $F\left(\lambda^{m+1}\right)=0$ in all the length preserving computations presented in the next section.

\section{Numerical Results}

Throughout the numerical experiments we take either $\vec{Y}^{0}=\overrightarrow{\mathcal{P}}_{Y}\left[\varsigma \pi^{h}\left[\vec{Q}^{0} \vec{\kappa}^{0}\right]\right]$, or, if $d=2, \vec{Y}^{0}=\overrightarrow{\mathcal{P}}_{Y}\left[\varsigma\left(\pi^{h}\left[\vec{Q}^{0} \vec{\kappa}^{0}\right]-\bar{\varkappa} \vec{\omega}^{0}\right)\right]$, where $\overrightarrow{\mathcal{P}}_{Y}$ is as in $(5.5 \mathrm{a}),(5.5 \mathrm{~b})$ and where in each case $\vec{\kappa}^{0} \in \underline{W}^{h}$ is an approximation of the curvature vector on $\Gamma^{0}$ defined by

$$
\left\langle\vec{\kappa}^{0}, \vec{\eta}\right\rangle_{\Gamma^{0}}^{h}+\left\langle\vec{X}_{s}^{0}, \vec{\eta}_{s}\right\rangle_{\Gamma^{0}}=0 \quad \forall \vec{\eta} \in \underline{W}^{h} .
$$

In practice, however, the numerical results are not very sensitive to the choice of $\vec{Y}^{0}$. Moreover, as we are mainly interested in (local) minimizers of the chosen elastic energies, i.e. in steady state solutions of the appropriate elastic flows, the particular choice of $\vec{Y}^{0}$ is mostly irrelevant. In the same vein we note that although in our derivations in Section 2 of elastic flows that involve enforcing Young's law at the junctions, e.g. the $C^{1}$ junction case and the nonlinear $C^{2}$ spline case, we assume that Young's law holds for the initial parameterization $\vec{x}(0)$, in most of our numerical experiments the polygonal curve parameterizations $\vec{X}^{0}$ will not satisfy Young's law.

Unless otherwise stated we choose $\varsigma_{i}=1, i=1 \rightarrow N$, and use the discretization parameters $J=100, \tau=10^{-3}$ and $T=2$. For evolutions without length constraints we always set $\lambda_{i}=0, i=1 \rightarrow N$. Unless otherwise stated the solutions $\vec{X}^{M}$ displayed in the following figures are always numerical steady states, in the sense that over several hundred time steps there is no noticable change in $\vec{X}^{m}$ or its discrete energy. Finally, we note that for better visualization any junction or boundary points are marked as red dots in the presentation of our numerical results. (We refer to the online version of this manuscript for figures in colour.)

\section{1. $C^{0}$ and $C^{1}$ double junctions}

Experiments for $C^{0}$ junctions without length constraints, where the initial curve is given by a unit circle, for varying values of $\bar{\varkappa} \in \mathbb{R}^{2}$, can be seen in Figures 3 and 4 , where the former show only symmetric evolutions. Of course, in the case $\bar{\varkappa}=(1,-1)$ the initial data is a steady state solution, and this is observed on the numerical level as well. Here, and in the remainder of this subsection, for our numerical results for $d=2$ we label curves from left to right and assume that they are parameterized in the direction from the upper junction to the lower junction. In the case $\bar{\varkappa}=(0,0)$ 


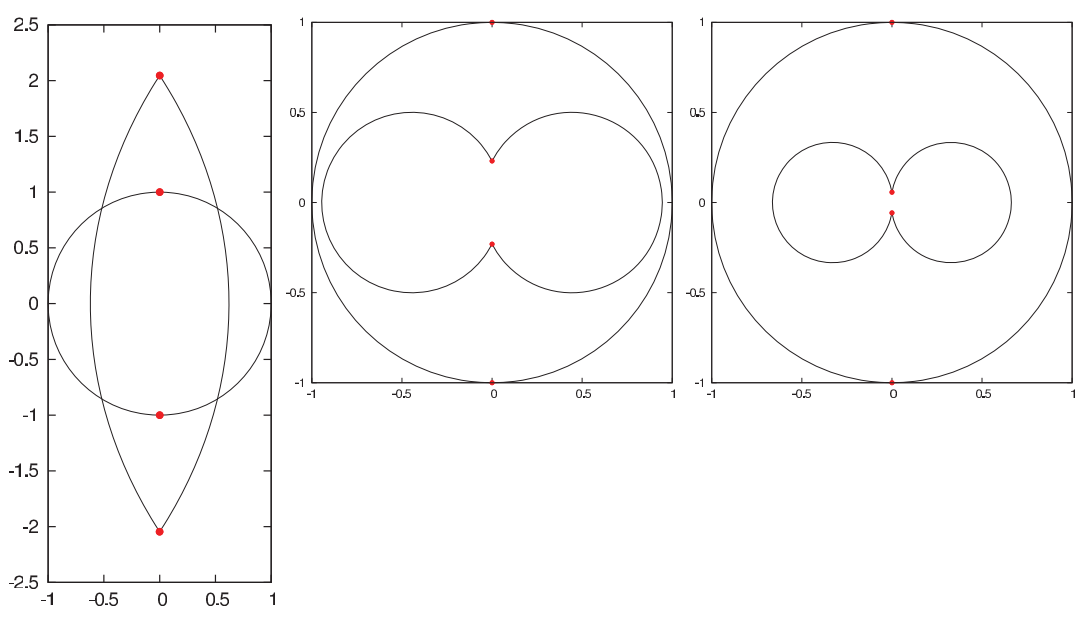

Fig. 3. $(d=2) C^{0}$ evolutions starting from a unit circle. The plots show $\vec{X}^{0}$ and $\vec{X}^{M}, \bar{\varkappa}=\left(\frac{1}{4},-\frac{1}{4}\right)$, $(2,-2),(3,-3)$.

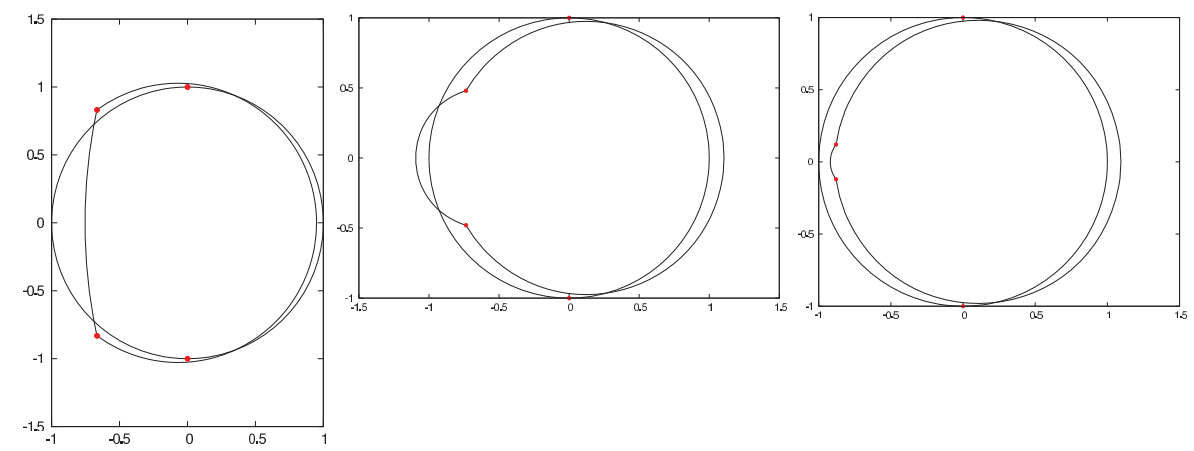

Fig. 4. $(d=2) C^{0}$ evolutions starting from a unit circle. The plots show $\vec{X}^{0}$ and $\vec{X}^{M}, \bar{\varkappa}=\left(\frac{1}{4},-1\right)$, $(2,-1),(5,-1)$.

no steady state is reached in finite time, with the two curves approaching a straight line segment and with the distance between the two junction points increasing in time.

The same experiments as in Figure 4, but now with a fully discrete approximation that maintains $\left|\Gamma_{i}^{m}\right|=\left|\Gamma_{i}^{0}\right|, i=1 \rightarrow 2$, exactly for all $m=1 \rightarrow M$, are shown in Figure 5. For the computation with $\bar{\varkappa}=(5,-1)$ we used the smaller time step $\tau=10^{-4}$.

Before we compare these results with the corresponding results from our scheme (4.6a), (4.6b) for the evolution of $C^{1}$ junctions, we present numerical evidence for the global equidistribution property of the semidiscrete approximation (3.36a), (3.36b) in the case $N=2$. To this end, we start with a partitioning of the unit circle, where 

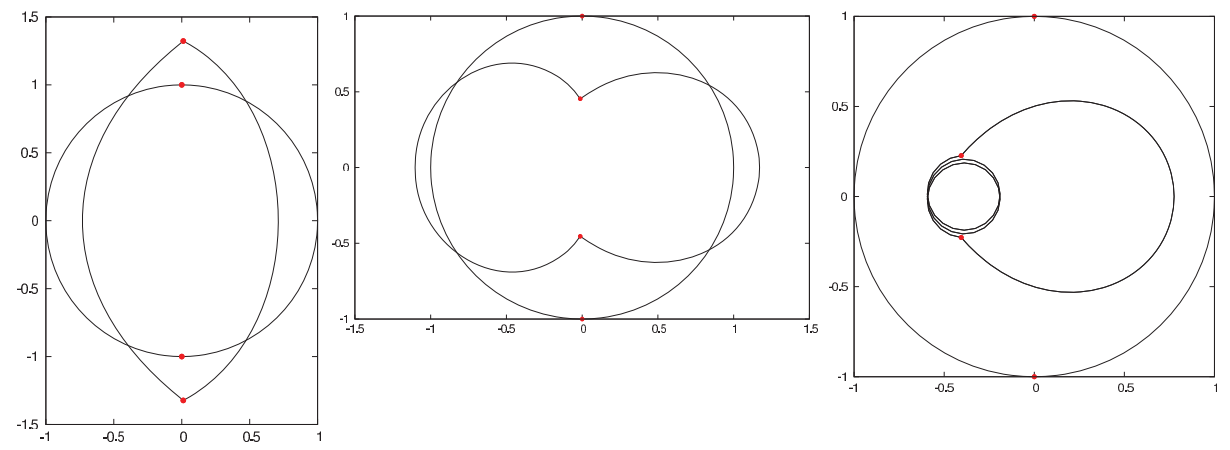

Fig. 5. $(d=2)$ Length preserving $C^{0}$ evolutions starting from a unit circle. The plots show $\vec{X}^{0}$ and $\vec{X}^{M}, \bar{\varkappa}=\left(\frac{1}{4},-1\right),(2,-1),(5,-1)$.
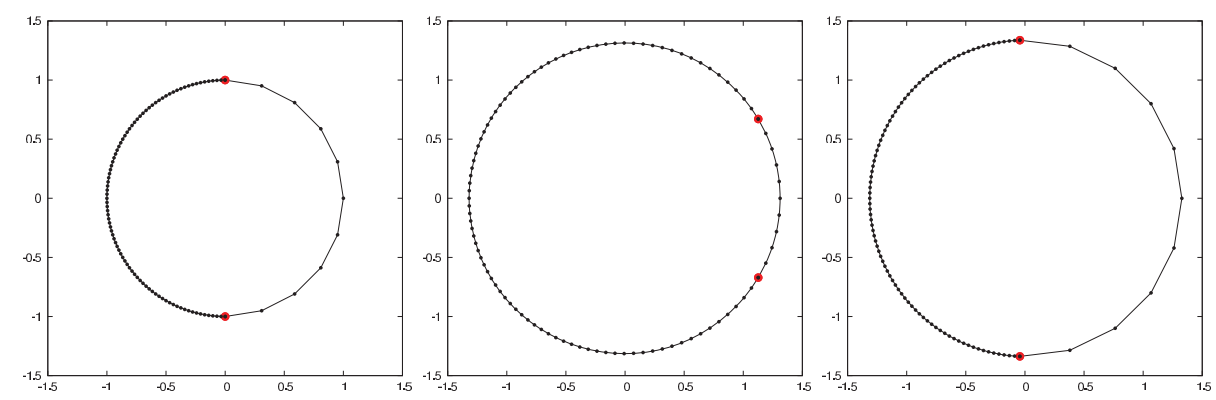

Fig. 6. $(d=2) C^{1}$ evolutions of a unit circle with $\bar{\varkappa}=(0,0)$. The plots show $\vec{X}^{0}$ (left) and $\vec{X}^{M}$ for a scheme with $\vec{Q}^{m}$ (middle) and with $\vec{Q}_{\star}^{m}$ (right).

$\Gamma_{1}^{0}$ has many more vertices than $\Gamma_{2}^{0}$. The results in Figure 6 show that for the fully discrete approximation based on (3.36a), (3.36b), the two junctions move tangentially in order to satisfy a global equidistribution property. For the scheme (4.5a), (4.5b), on the other hand, we have $\left|\Gamma_{1}^{m}\right| \approx\left|\Gamma_{2}^{m}\right|$ throughout, with each curve exhibiting an equidistributed partitioning. It is the undesirable global equidistribution property of the schemes (3.36a), (3.36b) and (3.37a), (3.37b) that made it necessary to introduce the more practical variants (3.48a), (3.48b) and (3.49a), (3.49b). The final time plots in Figure 6, for which we used $T=1$, are not numerical steady states, since the circle will continue to expand to decrease the elastic energy. We remark that both solutions in Figure 6 correspond to solutions of the continuous problem. In the case $\bar{\varkappa}=(0,0)$ with $\varsigma_{1}=\varsigma_{2}$ the problem with $C^{1}$ double junctions allows for several solutions as the junction points can move freely in tangential direction. A comparison of the results in Figure 5 with the corresponding results for $C^{1}$ junctions, using our scheme (4.6a), (4.6b), is shown in Figure 7. In addition, we repeat the last two sets of experiments for an initial setup, where $\Gamma_{1}(0)$ is still a unit semicircle, but now $\Gamma_{2}(0)$ is a $2: 1$ semiellipse. Here we computed until the 

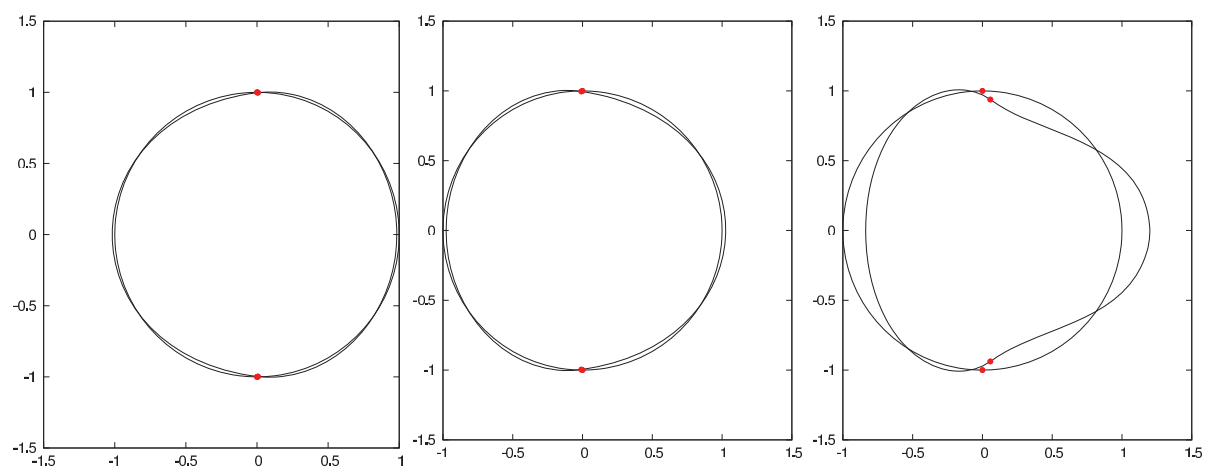

Fig. 7. $(d=2)$ Length preserving $C^{1}$ evolutions starting from a unit circle. The plots show $\vec{X}^{0}$ and $\vec{X}^{M}, \bar{\varkappa}=\left(\frac{1}{4},-1\right),(2,-1),(5,-1)$.
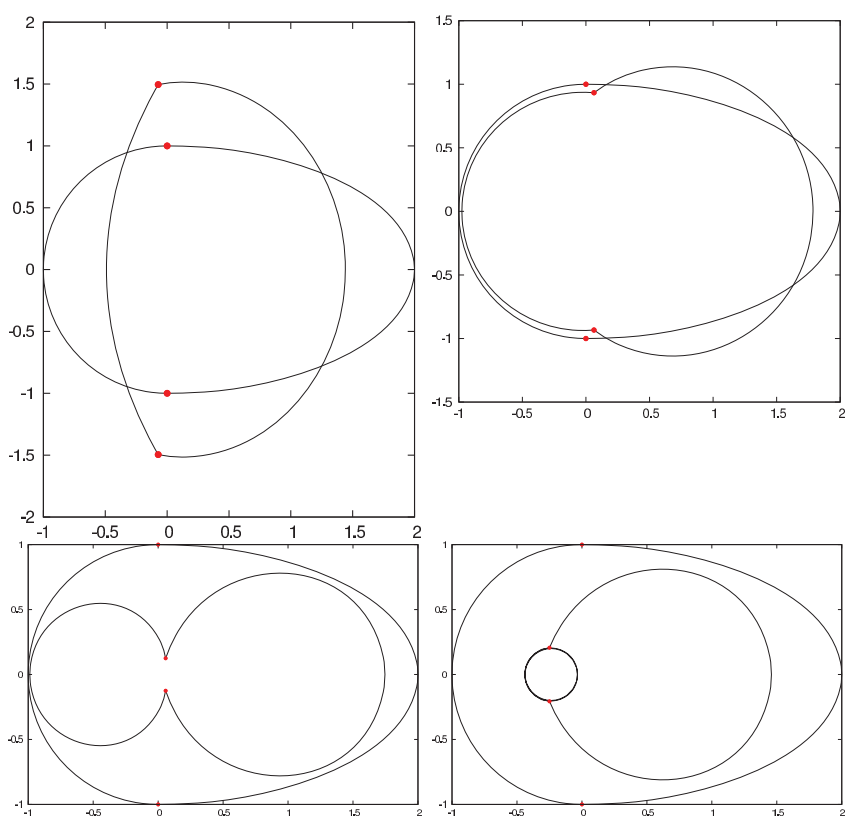

Fig. 8. $(d=2)$ Length preserving $C^{0}$ evolutions starting from a unit semicircle and a 2:1 semiellipse. The plots show $\vec{X}^{0}$ and $\vec{X}^{M}, \vec{\varkappa}=\left(\frac{1}{4},-1\right),(1,-1)(2,-1),(5,-1)$.

final time $T=5$. In addition, we used the smaller time step size $\tau=10^{-4}$ for the computations with $\bar{\varkappa}=(5,-1)$. The results can be seen in Figure 8 for the $C^{0}$ case and in Figure 9 for the $C^{1}$ case.

Finally we present some computations for our scheme (4.5a), (4.5b) with different values of $\varsigma \in \mathbb{R}_{>0}^{2}$. To this end we used the same initial setup as in Figure 9, and set again $T=5$. The results can be seen in Figure 10 . 


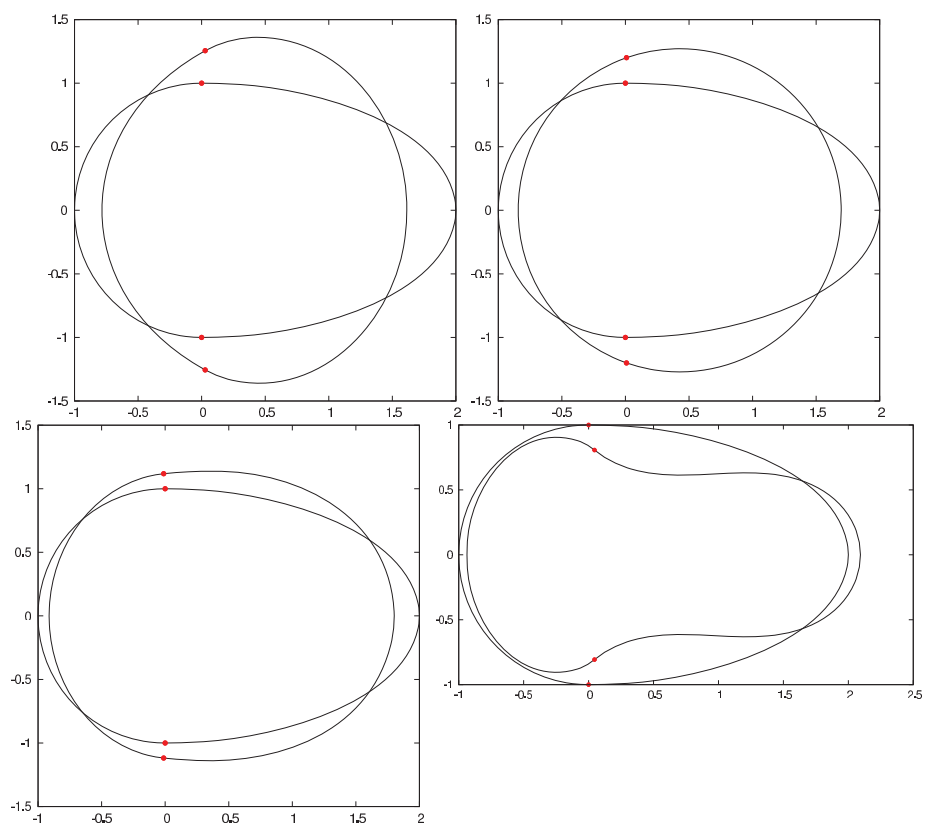

Fig. 9. $(d=2)$ Length preserving $C^{1}$ evolutions starting from a unit semicircle and a 2:1 semiellipse. The plots show $\vec{X}^{0}$ and $\vec{X}^{M}, \bar{\varkappa}=\left(\frac{1}{4},-1\right),(1,-1),(2,-1),(5,-1)$.

We do not present simulations in $\mathbb{R}^{3}$ for the case of two curves, since the steady state solutions will always lie within a two-dimensional hypersurface in $\mathbb{R}^{3}$.

\subsection{Young's law for triple and quadruple junctions}

Here we report on some computations where either three curves meet at two triple junctions or where four curves meet at two quadruple junctions, and where Young's law holds at the two junctions. The first set of experiments start from the unit standard double bubble. ${ }^{18}$. In the first experiment we set $\bar{\varkappa}=0$, and observe that the initial double bubble expands. However, it is not difficult to show that the two curved curves do not remain circle segments, i.e. the evolution is not self-similar in time. In the second experiment we set $\bar{\varkappa}=(0,-1,-1)$, for which we also present the numerical steady state for the approximation of the length preserving flow. Here, for the labelling and the parameterization of the curves, we use the same convention as in $\S 6.1$. We use $T=10$ for all three experiments and note that apart from the length preserving flow the final solutions displayed in Figure 11 are not numerical steady states.

An example for $d=3$ is shown in Figure 12. Here we replaced the straight line in the initial setup from Figure 11 with a circle arc that lies within the $\left(x_{2}, x_{3}\right)$-plane. As before, we set $T=10$. We observe that the solution continues to expand indefinitely. In particular, the solution converges to a self-similar profile which consists of 


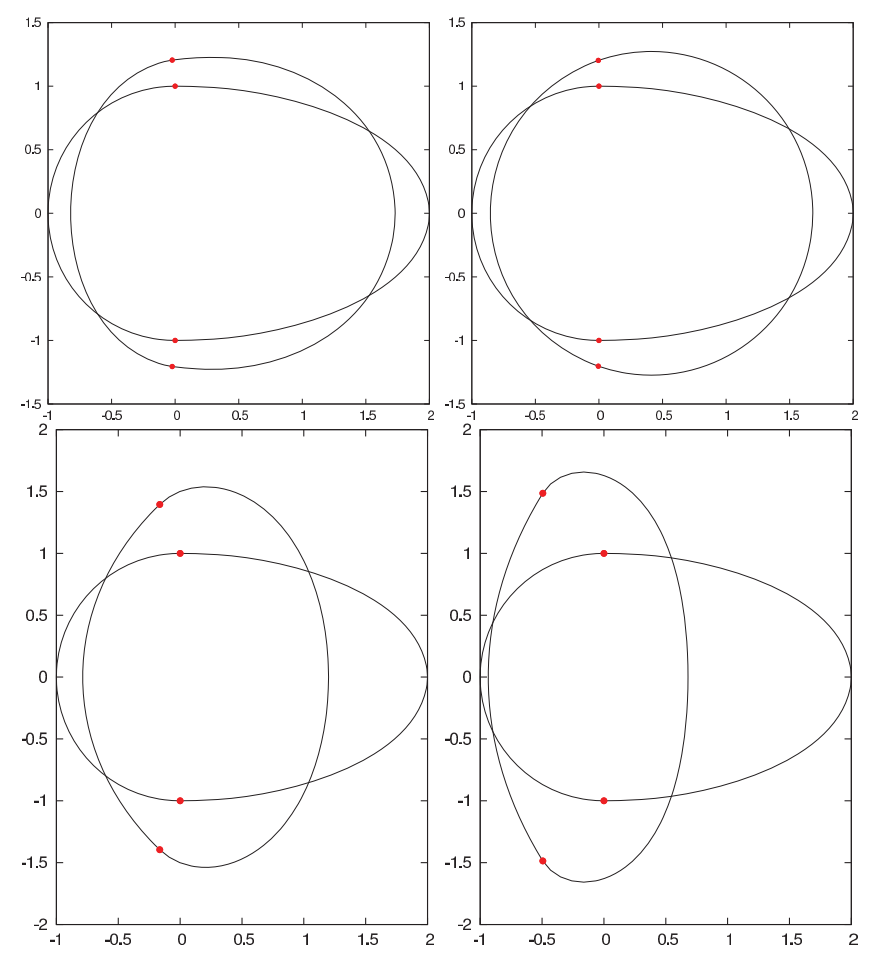

Fig. 10. $(d=2)$ Length preserving $C^{1}$ evolutions starting from a unit semicircle and a 2:1 semiellipse. The plots show $\vec{X}^{0}$ and $\vec{X}^{M}$ for $\bar{\varkappa}=(0,0)$ and $\varsigma=\left(\frac{1}{4}, 1\right),(1,1),(5,1),(10,1)$.

three semicircles which all lie on the same sphere and meet at two antipodal triple junctions with $120^{\circ}$ degree angles. In fact, a simple calculation shows that a true solution to $(2.6 \mathrm{a})$ with $(2.15 \mathrm{a})-(2.15 \mathrm{~d})$ and $\varsigma=(1,1,1)$ and $\lambda=(0,0,0)$ is given by

$$
\vec{x}_{i}(\rho, t)=(1+2 t)^{\frac{1}{4}}\left(\sin g(\rho) \cos \frac{2 i \pi}{3}, \sin g(\rho) \sin \frac{2 i \pi}{3}, \cos g(\rho)\right)^{T}, \quad i=1 \rightarrow 3 ;
$$

where $g(\rho)=\pi \rho+0.1 \sin (\pi \rho)$ in order to make the initial distribution of nodes non-uniform. We use the true solution (6.2) for a convergence test for our finite element approximation. Here we compute the error $\|\vec{X}-\vec{x}\|_{L^{\infty}}:=\max _{m=1 \rightarrow M}\left\|\vec{X}^{m}-\vec{x}\left(\cdot, t_{m}\right)\right\|_{L^{\infty}}$, where $\left\|\vec{X}^{m}-\vec{x}\left(\cdot, t_{m}\right)\right\|_{L^{\infty}}:=$ $\max _{i=1 \rightarrow 3} \max _{j=1 \rightarrow J_{i}} \min _{\rho \in[0,1]}\left|\vec{X}_{i}^{m}\left(q_{j}^{i}\right)-\vec{x}_{i}\left(\rho, t_{m}\right)\right|$, between $\vec{X}$ and the true solution $\vec{x}$ on the interval $[0, T]$. The numbers in Table 6.2 , where we report on the errors for $T=1$ and $\tau=0.5 h^{2}$, indicate a convergence rate for the error of $O\left(h^{2}\right)$.

Two examples for the elastic flow of quadruple junctions with Young's law are shown in Figure 13. In each case, the network assumes the shape of two circles that intersect at the two quadruple junctions with angles that satisfy (2.16c). Here we note that the condition (2.16c), in contrast to the case $N=3$, for $N=4$ does not fix the angles at the junction. In the first example in Figure 13, the observed angles 


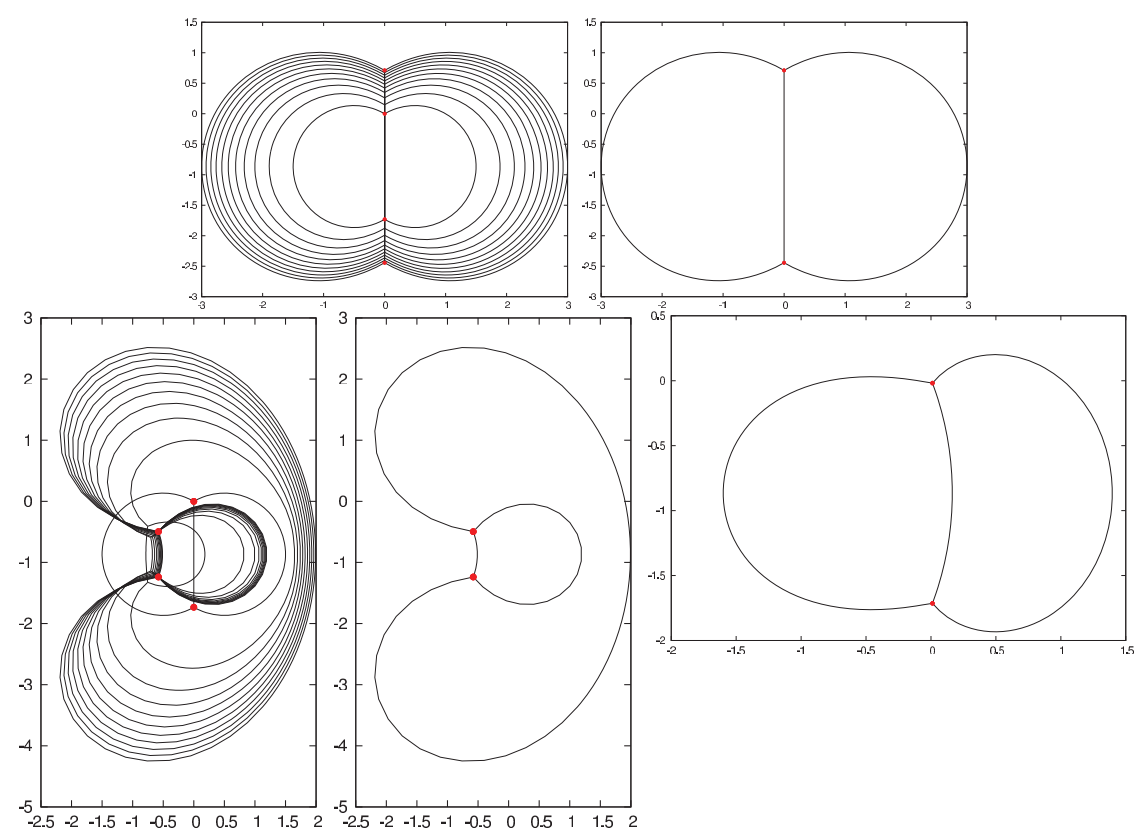

Fig. 11. $(d=2)$ Triple junction evolutions with Young's law. The top row shows $\vec{X}^{m}$ at times $t_{m}=0,1, \ldots, 10$, and $\vec{X}^{M}$ for $\bar{\varkappa}=(0,0,0)$. The bottom row shows $\vec{X}^{m}$ at times $t_{m}=0,1, \ldots, 10$, and $\vec{X}^{M}$ for $\bar{\varkappa}=(0,-1,-1)$. In addition, we show $\vec{X}^{M}$ for the length preserving flow.
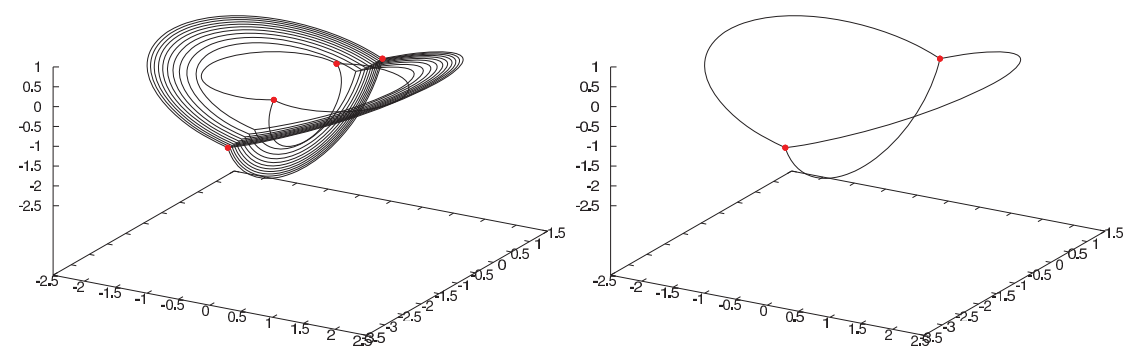

Fig. 12. $(d=3)$ Triple junction evolutions with Young's law. The plots show $\vec{X}^{m}$ at times $t_{m}=$ $0,1, \ldots, 10$, and $\vec{X}^{M}$.

at time $t=T$ are $\left(37^{\circ}, 138^{\circ}, 37^{\circ}, 148^{\circ}\right)$. As the two circles keep on expanding, the displayed solution is not a steady state. Moreover, the observed angles clearly do not satisfy (2.16c) exactly. This is due to discretization errors and due to the fact the our schemes approximate (2.16c) weakly. For the second experiment, on the other hand, we obtain a numerical steady state that is given by two intersecting circles with radii 1 and 2 . In this case the angles at the two quadruple junctions are given by $\left(36^{\circ}, 137^{\circ}, 40^{\circ}, 147^{\circ}\right)$. Once again, they only satisfy $(2.16 \mathrm{c})$ approximatively. Using finer space and time discretization parameters would lead to a better approximation 
Table 1. Absolute errors for the test problem with estimated order of convergence.

\begin{tabular}{rcc}
\hline \hline$J$ & $\|\vec{X}-\vec{x}\|_{L^{\infty}}$ & eоc \\
\hline 30 & $1.2651 \mathrm{e}-02$ & - \\
60 & $2.7189 \mathrm{e}-03$ & 2.218156 \\
120 & $6.2105 \mathrm{e}-04$ & 2.130242 \\
240 & $1.4853 \mathrm{e}-04$ & 2.063955 \\
480 & $3.6611 \mathrm{e}-05$ & 2.020405 \\
960 & $9.1765 \mathrm{e}-06$ & 1.996261 \\
\hline
\end{tabular}
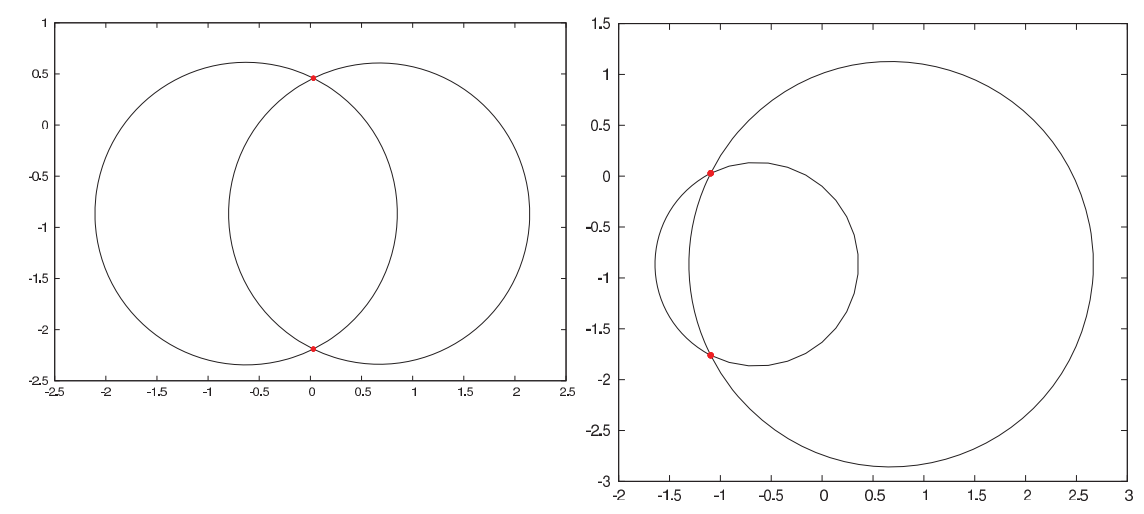

Fig. 13. $(d=2)$ Quadruple junction solutions $\vec{X}^{M}$ with Young's law. Here $\bar{\varkappa}=(0,0,0,0)$ and $\bar{\varkappa}=\left(1, \frac{1}{2},-\frac{1}{2},-1\right)$.

of the correct angles, similarly to the numerical results in Table 3 in Ref. 1.

\subsection{Nonlinear $C^{2}$ splines}

First we compute examples for a closed nonlinear spline, with $N=2$. Using the same initial data as in Figure 3, i.e. two unit semicircles, we compute several nonlinear spline examples with varying values of $\bar{\varkappa}$. Note that for the numerical results in this subsection the closed planar chains of curves are assumed to be parameterized anti-clockwise. For the results in Figure 14, we choose $\bar{\varkappa}_{1}=-\bar{\varkappa}_{2}$, so that the curves try to attain a banana kind of shape. Recall from Remark 2.8 that in this case the approximated curves are only of type $C^{1}$ at the junctions. We set $T=1000$ for the experiment with $\bar{\varkappa}=\left(\frac{1}{4},-\frac{1}{4}\right)$, and $T=10$ otherwise, so that in each case the displayed final solution is a numerical steady state. A computation for a closed spline with $N=3$ is shown in Figure 15 . Here $\bar{\varkappa}=(-2,2,2)$, while the initial setup is given as a unit semicircle and two unit quartercircles.

The remainder of the computations presented in this subsection are for the scheme (4.7a), (4.7b), i.e. we compute open nonlinear $C^{2}$ splines with clamped boundary conditions at the two endpoints. Throughout we set $\vec{\zeta}_{0}=-\vec{\zeta}_{N}=$ 

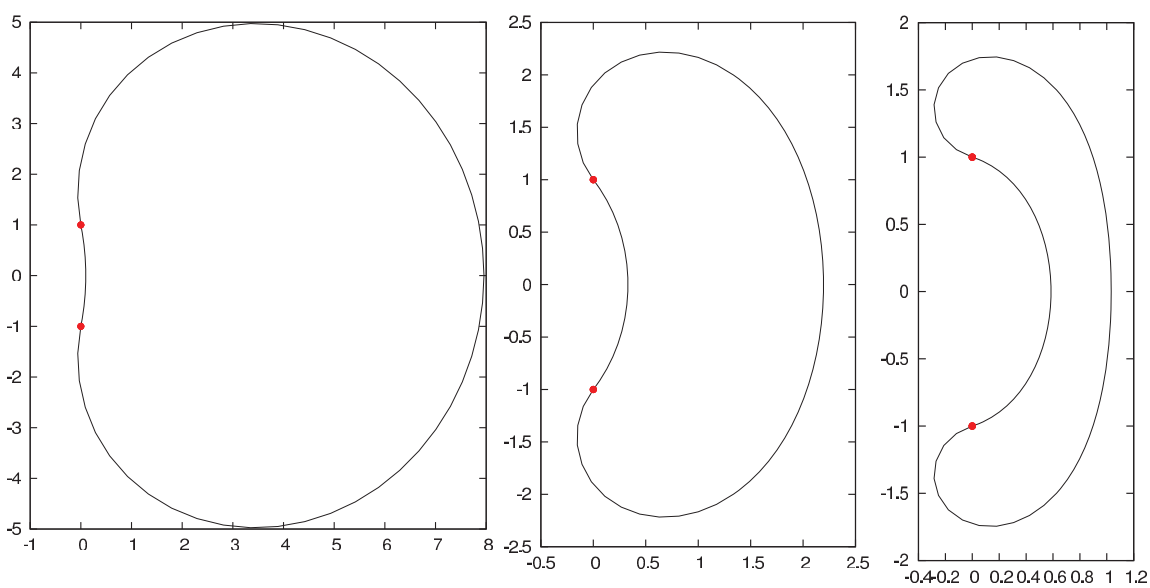

Fig. 14. $(d=2)$ Nonlinear $C^{1}$ spline solutions $\vec{X}^{M}$ for $\bar{\varkappa}=\left(\frac{1}{4},-\frac{1}{4}\right),(1,-1),(2,-2)$.

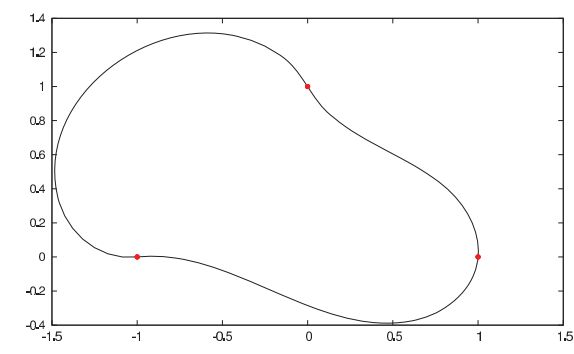

Fig. 15. $(d=2)$ Nonlinear $C^{1}$ spline solution $\vec{X}^{M}$. Here $\bar{\varkappa}=(-2,2,2)$.

$(1,0, \ldots, 0)^{T} \in \mathbb{R}^{d}$. In each case the initial curve $\Gamma^{0}$ is given by straight line segments that connect the given spline node points $\left\{\vec{\alpha}_{l}\right\}_{l=0}^{N}$. As before, the spline node points $\left\{\vec{\alpha}_{l}\right\}_{l=0}^{N}$ are clearly visible as red dots in our graphical plots. The two computations in Figure 16 have $N=2$ and $N=3$, respectively. The former shows a steady state solution, while the latter curve would continue to expand in order to decrease the elastic energy further. For the second experiment we chose the smaller time step size $\tau=10^{-4}$. A first result for $N=3$ and $d=3$ is shown on the left of Figure 17, where the shown solution is once again not a steady state, since the curve can lower its elastic energy by expanding further. If two further spline node points are added, then we obtain the shape on the right of Figure 17. This is now a numerical steady state solution. For both experiments in Figure 17 we set $\tau=10^{-4}$. Finally, we present results for $d=2$ with $N=7$ and $N=9$, and for $d=3$ with $N=10$. The solutions shown in Figures 18 and 19 are all numerical steady states. 


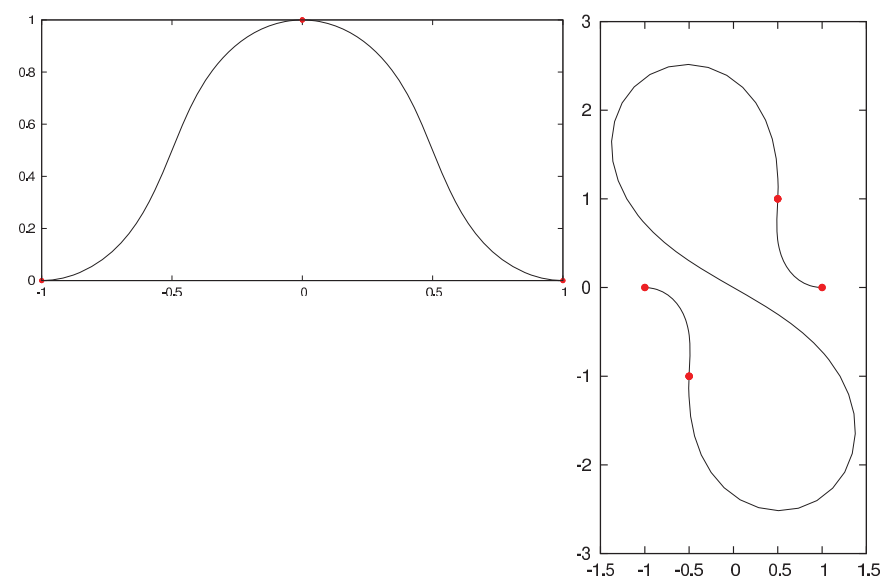

Fig. 16. $(d=2)$ Nonlinear $C^{2}$ spline solutions $\vec{X}^{M}$.
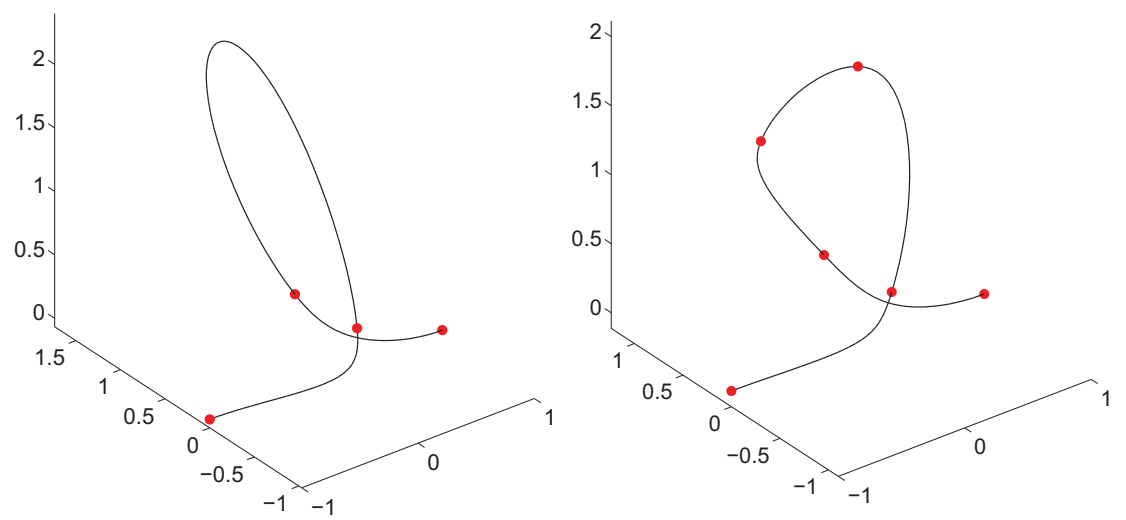

Fig. 17. $(d=3)$ Nonlinear $C^{2}$ spline solutions $\vec{X}^{M}$.

\section{References}

1. J. W. Barrett, H. Garcke and R. Nürnberg, On the variational approximation of combined second and fourth order geometric evolution equations, SIAM J. Sci. Comput. 29 (2007) 1006-1041.

2. J. W. Barrett, H. Garcke and R. Nürnberg, A parametric finite element method for fourth order geometric evolution equations, J. Comput. Phys. 222 (2007) 441-462.

3. J. W. Barrett, H. Garcke and R. Nürnberg, Parametric approximation of Willmore flow and related geometric evolution equations, SIAM J. Sci. Comput. 31 (2008) $225-253$.

4. J. W. Barrett, H. Garcke and R. Nürnberg, Numerical approximation of gradient flows for closed curves in $\mathbb{R}^{d}$, IMA J. Numer. Anal. 30 (2010) 4-60. 

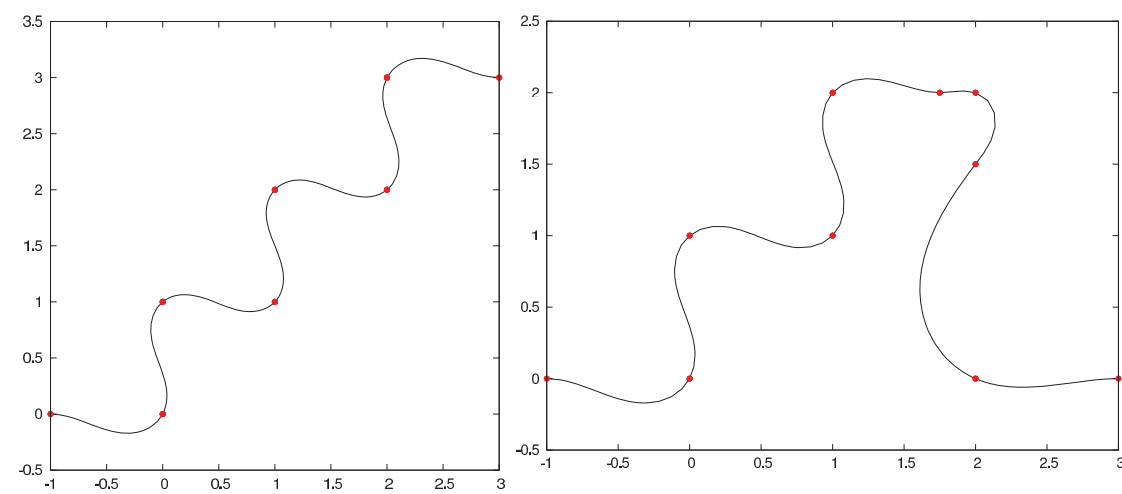

Fig. 18. $(d=2)$ Nonlinear $C^{2}$ spline solutions $\vec{X}^{M}$.
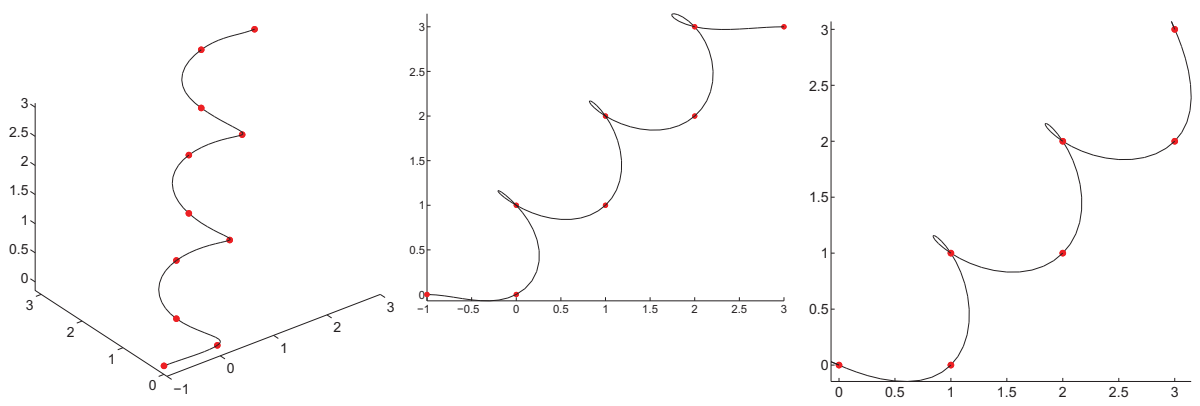

Fig. 19. $(d=3)$ Nonlinear $C^{2}$ spline solution $\vec{X}^{M}$ from three different viewpoints.

5. J. W. Barrett, H. Garcke and R. Nürnberg, Parametric approximation of surface clusters driven by isotropic and anisotropic surface energies, Interfaces Free Bound. 12 (2010) 187-234.

6. J. W. Barrett, H. Garcke and R. Nürnberg, The approximation of planar curve evolutions by stable fully implicit finite element schemes that equidistribute, Numer. Methods Partial Differential Equations 27 (2011) 1-30.

7. J. W. Barrett, H. Garcke and R. Nürnberg, Parametric approximation of isotropic and anisotropic elastic flow for closed and open curves, Numer. Math. (to appear).

8. G. Birkhoff and C. R. de Boor, Piecewise polynomial interpolation and approximation, in Approximation of Functions (Proc. Sympos. General Motors Res. Lab., 1964) (Elsevier Publ. Co., Amsterdam, 1965), pp. 164-190.

9. T. A. Davis, Algorithm 915 SuiteSparseQR: Multifrontal multithreaded rank-revealing sparse QR factorization, ACM Trans. Math. Software 38, (to appear).

10. K. Deckelnick and G. Dziuk, Error analysis for the elastic flow of parametrized curves, Math. Comp. 78 (2009) 645-671.

11. K. Deckelnick, G. Dziuk and C. M. Elliott, Computation of geometric partial differential equations and mean curvature flow, Acta Numer. 14 (2005) 139-232.

12. G. Dziuk, E. Kuwert and R. Schätzle, Evolution of elastic curves in $\mathbb{R}^{n}$ : existence and computation, SIAM J. Math. Anal. 33 (2002) 1228-1245. 
13. S. Esedoglu, S. J. Ruuth and R. Tsai, Threshold dynamics for high order geometric motions, Interfaces Free Bound. 10 (2008) 263-282.

14. H. Garcke and A. Novick-Cohen, A singular limit for a system of degenerate CahnHilliard equations, Adv. Differential Equations 5 (2000) 401-434.

15. M. Golomb and J. Jerome, Equilibria of the curvature functional and manifolds of nonlinear interpolating spline curves, SIAM J. Math. Anal. 13 (1982) 421-458.

16. W. Helfrich, Elastic properties of lipid bilayers: Theory and possible experiments, $Z$. Naturforsch. 28c (1973) 693-703.

17. M. Helmers, Snapping elastic curves as a one-dimensional analogue of two-component lipid bilayers, Math. Models Methods Appl. Sci. 21 (2011) 1027-1042.

18. M. Hutchings, F. Morgan, M. Ritoré and A. Ros, Proof of the double bubble conjecture, Ann. of Math. (2) 155 (2002) 459-489.

19. R. L. Levien, From spiral to spline: Optimal techniques in interactive curve design, Ph.D. thesis, University of California, Berkeley, 2009.

20. J. S. Lowengrub, A. Rätz and A. Voigt, Phase-field modeling of the dynamics of multicomponent vesicles: spinodal decomposition, coarsening, budding, and fission, Phys. Rev. E 79 (2009) 0311926.

21. E. Mehlum, Nonlinear splines, in Computer aided geometric design (Proc. Conf., Univ. Utah, Salt Lake City, Utah, 1974), eds. R. E. Barnhill and R. F. Riesenfeld (Academic Press, New York, 1974), pp. 173-207.

22. W. Mio, A. Srivastava and E. Klassen, Interpolations with elasticae in Euclidean spaces, Quart. Appl. Math. 62 (2004) 359-378.

23. H. P. Moreton, Minimum curvature variation curves, networks, and surfaces for fair free-form shape design, Ph.D. thesis, University of California, Berkeley, 1993.

24. H. P. Moreton and C. H. Sequin, Scale-invariant minimum-cost curves: Fair and robust design implements, Comput. Graph. Forum 12 (1993) 473-484.

25. D. Mumford, Elastica and computer vision, in Algebraic geometry and its applications (West Lafayette, IN, 1990) (Springer, New York, 1994), pp. 491-506.

26. F. Tröltzsch, Optimal Control of Partial Differential Equations: Theory, Methods and Applications, volume 112 of Graduate Studies in Mathematics (American Mathematical Society, Providence, RI, 2010).

27. C. Truesdell, The influence of elasticity on analysis: the classic heritage, Bull. Amer. Math. Soc. (N.S.) 9 (1983) 293-310. 